\title{
Convergence Rates in the Law of Large Numbers for Arrays of Banach Valued Martingale Differences
}

\begin{abstract}
Shunli Hao
Trade and Event Management, School of Economics, Beijing International Studies University, Beijing 100024, China

Correspondence should be addressed to Shunli Hao; hsl2005100@163.com

Received 15 July 2013; Accepted 18 September 2013

Academic Editor: Dumitru Motreanu

Copyright ( 2013 Shunli Hao. This is an open access article distributed under the Creative Commons Attribution License, which permits unrestricted use, distribution, and reproduction in any medium, provided the original work is properly cited.

We study the convergence rates in the law of large numbers for arrays of Banach valued martingale differences. Under a simple moment condition, we show sufficient conditions about the complete convergence for arrays of Banach valued martingale differences; we also give a criterion about the convergence for arrays of Banach valued martingale differences. In the special case where the array of Banach valued martingale differences is the sequence of independent and identically distributed real valued random variables, our result contains the theorems of Hsu-Robbins-Erdös (1947, 1949, and 1950), Spitzer (1956), and Baum and Katz (1965). In the real valued single martingale case, it generalizes the results of Alsmeyer (1990). The consideration of Banach valued martingale arrays (rather than a Banach valued single martingale) makes the results very adapted in the study of weighted sums of identically distributed Banach valued random variables, for which we prove new theorems about the rates of convergence in the law of large numbers. The results are established in a more general setting for sums of infinite many Banach valued martingale differences. The obtained results improve and extend those of Ghosal and Chandra (1998).
\end{abstract}

\section{Introduction}

The convergence rates in the law of large numbers have been considered by many authors. Let $\left(X_{j}\right)_{j \geq 1}$ be a sequence of independent and identically distributed (i.i.d.) real valued random variables defined on a probability space $(\Omega, \mathscr{F}, \mathbb{P})$ with $\mathbb{E} X_{i}=0$, and set $S_{n}=\sum_{j=1}^{n} X_{j}$. By the law of large numbers, $\mathbb{P}\left\{\left|S_{n}\right|>\varepsilon n\right\} \rightarrow 0$ for $\varepsilon>0$. Hsu and Robbins [1] introduced the notion of complete convergence and showed that

$$
\sum_{n=1}^{\infty} \mathbb{P}\left\{\left|S_{n}\right|>\varepsilon n\right\}<\infty \quad \forall \varepsilon>0
$$

if $\mathbb{E} X_{1}^{2}<\infty$; Erdös [2,3] proved that the converse also holds. Spitzer [4] showed that

$$
\sum_{n=1}^{\infty} n^{-1} \mathbb{P}\left\{\left|S_{n}\right|>\varepsilon n\right\}<\infty \quad \forall \varepsilon>0
$$

whenever $\mathbb{E} X_{1}=0$. Katz [5] and Baum and Katz [6] proved that, for $p=1 / \alpha$ and $\alpha \geq 1 / 2$, or $p>1 / \alpha$ and $\alpha>1 / 2$,

$$
\sum_{n=1}^{\infty} n^{p \alpha-2} \mathbb{P}\left\{\left|S_{n}\right|>\varepsilon n^{\alpha}\right\}<\infty \quad \forall \varepsilon>0
$$

if and only if $\mathbb{E}\left|X_{1}\right|^{p}<\infty$. Lai [7] studied the limiting case where $p>2$ and $\alpha=1 / 2$. Gafurov and Slastnikov [8] considered the case where $\left(n^{p \alpha-2}\right)$ and $\left(n^{\alpha}\right)$ are replaced by more general sequences. Many authors have considered the generalization of the theorem of Baum and Katz [6] to arrays of independent (but not necessarily identically distributed) random variables; see for example Li et al. [9], Hu et al. [1012], Kuczmaszewska [13], Sung et al. [14], and Kruglov et al. [15].

Let $\left(X_{j}\right)_{j \geq 1}$ be a sequence of real-valued martingale differences defined on a probability space $(\Omega, \mathscr{F}, \mathbb{P})$, adapted to a filtration $\left(\mathscr{F}_{j}\right)$, with $\mathscr{F}_{0}=\{\emptyset, \Omega\}$. This means that for each (integer) $j \geq 1, X_{j}$ is $\mathscr{F}_{j}$-measurable and 
$\mathbb{E}\left[X_{j} \mid \mathscr{F}_{j-1}\right]=0$ a.s. A natural question is whether the prementioned theorem of Baum and Katz [6] is still valid for martingale differences $\left(X_{j}\right)$. Lesigne and Volný [16] proved that, for $p \geq 2$, sup $_{j \geq 1} \mathbb{E}\left|X_{j}\right|^{p}<\infty$ implies

$$
\mathbb{P}\left(\left|S_{n}\right|>\varepsilon n\right)=O\left(n^{-p / 2}\right)
$$

(as usual, we write $a_{n}=o\left(b_{n}\right)$ if $\lim _{n \rightarrow \infty} a_{n} / b_{n}=0$ and $a_{n}=O\left(b_{n}\right)$ if the sequence $\left(a_{n} / b_{n}\right)$ is bounded) and that the exponent $p / 2$ is the best possible, even for strictly stationary and ergodic sequences of martingale differences. Therefore, the theorem of Baum and Katz does not hold for martingale differences without additional conditions. (Stoica [17] claimed that the theorem of Baum and Katz still holds for $p>2$ in the case of martingale differences without additional assumption, but his claim is a contradiction with the conclusion of Lesigne and Volný [16], and his proof contains an error: when $p>2$, we cannot choose $\alpha$ satisfying (6) of [17].) Alsmeyer [18] proved that the theorem of Baum and Katz for $p>1 / \alpha$ and $1 / 2<\alpha \leq 1$ still holds for martingale differences $\left(X_{j}\right)_{j \geq 1}$ if for some $\gamma \in(1 / \alpha, 2]$ and $q \in[1, \infty]$ with $q>(p \alpha-1) /(\gamma \alpha-1)$,

$$
\sup _{n \geq 1}\left\|\frac{1}{n} \sum_{j=1}^{n} \mathbb{E}\left[\left|X_{j}\right|^{\gamma} \mid \mathscr{F}_{j-1}\right]\right\|_{q}<\infty,
$$

where $\|\cdot\|_{q}$ denotes the $L^{q}$ norm. This is a nice result; nevertheless, it is not always satisfied in applications; for example, (a) it does not apply to "nonhomogeneous" cases, such as martingales of the form $S_{n}=\sum_{j=1}^{n} j^{a} Y_{j}$, where $a>$ 0 and $Y_{j}$ are i.i.d., as in this case the condition (5) (with $X_{j}=j^{a} Y_{j}$ ) is never satisfied; (b) in applications instead of a single martingale we often need to consider martingale arrays: for example, when we use the decomposition of a random sequence $\left(S_{n}\right)$ into martingale differences (such as in the study of directed polymers in a random environment), the summands usually depend on $n: S_{n}=\sum_{j=1}^{n} X_{n j}, X_{n j}=\mathbb{E}\left[S_{n} \mid\right.$ $\left.\mathscr{F}_{j}\right]-\mathbb{E}\left[S_{n} \mid \mathscr{F}_{j-1}\right]$, where $\mathscr{F}_{0}=\{\emptyset, \Omega\}$ and $\mathscr{F}_{i}=\sigma\left(S_{1}, \ldots, S_{i}\right)$ for $i \geq 1$.

Our first main objective is to extend the theorem of Baum and Katz [6] to a large class of Banach valued martingale arrays. More precisely, under a simple moment condition on $\sum_{j=1}^{n} \mathbb{E}\left[\left\|X_{n j}\right\|^{\gamma} \mid \mathscr{F}_{n, j-1}\right]$ for some $\gamma \in(1,2]$, we will find sufficient conditions for

$$
\sum_{n=1}^{\infty} \phi(n) \mathbb{P}\left\{\left\|S_{n, \infty}\right\|>\varepsilon\right\}<\infty
$$

for a large class of sequences of Banach valued martingale differences $\left\{\left(X_{n j}, \mathscr{F}_{n j}\right)\right\}_{j \geq 1}, n \geq 1$, where $S_{n, \infty}=\sum_{j=1}^{\infty} X_{n j}$, $\phi: \mathbb{N} \rightarrow[0, \infty)$ is a positive function, and $\varepsilon>0$. Of particular interest is the case where $\phi(n)$ is a regular function: $\phi(n)=n^{b-1} l(n)(b \geq 0), l(\cdot)>0$ being slowly varying at $\infty$; that is, $l(\cdot)$ is a positive measurable function defined on $(0, \infty)$ such that $\lim _{x \rightarrow \infty}(l(\lambda x) / l(x))=1$ for any $\lambda>0$. Our results improve and complete a result of Ghosal and Chandra [19] for martingale arrays and extend Alsmeyer's result [18] for martingales.
Our second main objective is to extend another important theorem of Baum and Katz [6] which states that for i.i.d. real valued random variables $X_{j}$ with $\mathbb{E} X_{j}=0$ and for each $p \geq 1$, $\mathbb{P}\left(\left|X_{1}\right|>n\right)=o\left(n^{-p}\right)$ if and only if $\mathbb{P}\left(\left|S_{n}\right|>\varepsilon n\right)=o\left(n^{-(p-1)}\right)$ for all $\varepsilon>0$. In fact, we prove that a similar result holds for a large class of Banach valued martingale arrays: under a simple moment condition on $\sum_{j=1}^{n} \mathbb{E}\left[\left\|X_{n j}\right\|^{\gamma} \mid \mathscr{F}_{n, j-1}\right]$ for some $\gamma \in$ $(1,2]$, we obtain sufficient conditions for

$$
\phi(n) \mathbb{P}\left\{\left\|S_{n, \infty}\right\|>\varepsilon\right\}=o(1) \quad(\text { resp., } O(1)),
$$

where $\phi$ and $S_{n, \infty}$ are defined as before, $\varepsilon>0$. The result is new and sharp even for independent but not identically distributed real valued random variables.

The consideration of a Banach valued martingale array (rather than a Banach valued single martingale) makes our results very adapted in the study of weighted sums of identically distributed Banach valued random variables. Many authors have contributed to this subject. Gut [20], Lanzinger and Stadtmüller [21] considered weighted sums of i.i.d. random variables. Li et al. [9], Wang et al. [22] studied weighted sums of independent random variables. $\mathrm{Yu}$ [23] considered weighted sums of martingale differences (see also the references therein). Ghosal and Chandra [19] considered weighted sums of arrays of martingale differences. As applications of our main results, we generalize or improve some of their results. For example, we prove a new theorem about the convergence rate for weighted sums of identically distributed Banach valued martingale differences.

As information, we mention that Baum-Katz type theorems in different dependent setups have been studied by many authors. For example, Li et al. [24] studied moving average processes; Shao [25, 26], Szewczak [27] considered mixing conditions; Baek and Park [28] studied negatively dependent random variables; Liang [29], Liang and Su [30], Kuczmaszewska [31], Kruglov [32], and Ko [33] studied negatively associated random variables.

The rest of the paper is organized as follows. In Section 2, we establish some maximal inequalities for Banach valued martingales. In Section 3, we show our main results on the convergence rates for Banach valued martingale arrays, which improve and complete Theorem 2 of Ghosal and Chandra [19]. In Section 4, we consider the important special case of triangular Banach valued martingale arrays, and obtain an extension of Theorem 1 and 2 of Alsmeyer [18]. We also generalize a result of Chow and Teicher (cf. [34, page 393]) about the complete convergence of sums of independent real valued random variables. In Section 5, we look for the convergence rates for the maxima of sequences of any Banach valued random variables, in order to obtain further equivalent conditions about the convergence rates for Banach valued martingales in the following section. In Section 6, we consider the convergence rates for Banach valued martingales. Our results extend Theorems 1-4 of Baum and Katz [6] for i.i.d. real valued random variables and generalize Theorems 1 and 2 of Alsmeyer [18]. As applications, in Section 7, we obtain new results on the convergence rates for weighted sums of Banach valued martingale differences, which extend Theorems 2 and 3 of Lanzinger and Stadtmüller [21] on weighted sums of 
the form $\sum_{j=1}^{n} j^{a-1} X_{j}$. In Section 8, we consider more general weighted sums of Banach valued martingale differences, for which we extend Theorem 3.3 of Baxter et al. [35], Corollary 1 of Ghosal and Chandra [19], and Theorems 2.2-2.4 of Li et al. [9] and generalize Theorem 2 of $\mathrm{Yu}$ [23].

For notations, as usual, we write $\mathbb{N}^{*}=\{1,2, \ldots\}, \mathbb{N}=$ $\{0\} \bigcup \mathbb{N}^{*}$ and $\mathbb{R}=(-\infty, \infty)$.

\section{Maximal Inequalities for Banach Valued Martingales}

In this section, we show new maximal inequalities for Banach valued martingales.

Let $(\Omega, \mathscr{F}, \mathbb{P})$ be a probability space and $(\mathbb{B},\|\cdot\|)$ a real separable Banach space. For any real number $p \geq 1$, denote by $\mathbb{L}_{\mathbb{B}}^{p}$ the space of $\mathbb{B}$-valued random variables such that $\|X\|_{\mathbb{L}_{\mathbb{B}}^{p}}=$ $\left(\mathbb{E}\|X\|^{p}\right)^{1 / p}$ is finite. Let $\mathscr{F}_{0}=\{\emptyset, \Omega\} \subset \mathscr{F}_{1} \subset \cdots$ be an increasing sequence of sub- $\sigma$-fields of $\mathscr{F}$. Let $\left\{\left(X_{j}, \mathscr{F}_{j}\right)\right\}_{j=1}^{n}$ be an adapted sequence of $\mathbb{B}$-valued random variables defined on $(\Omega, \mathscr{F}, \mathbb{P})$; that is, for every $j \geq 1, X_{j}$ is $\mathscr{F}_{j}$ measurable. We call it a sequence of $\mathbb{B}$-valued martingale differences if additionally $\mathbb{E}\left[X_{j} \mid \mathscr{F}_{j-1}\right]=0$ a.s. and $X_{j}$ belongs to $\mathbb{L}_{\mathbb{B}}^{1}$ for any $j \geq 1$ and a sequence of $\mathbb{B}$-valued supermartingale differences if additionally $\mathbb{E}\left[X_{j} \mid \mathscr{F}_{j-1}\right] \leq 0$ a.s. and $X_{j}$ belongs to $\mathbb{L}_{\mathbb{B}}^{1}$ for any $j \geq 1$. Following Pisier [36], we say that a Banach space $(\mathbb{B},\|\cdot\|)$ is $\gamma$-smooth $(1<\gamma \leq 2)$ if there exists an equivalent norm $\|\cdot\|_{\mathbb{B}}$ such that

$$
\begin{aligned}
\sup _{t>0} & \left\{\frac{1}{t^{\gamma}} \sup \left\{\|x+t y\|_{\mathbb{B}}+\|x-t y\|_{\mathbb{B}}-2:\|x\|_{\mathbb{B}}=\|y\|_{\mathbb{B}}=1\right\}\right\} \\
& <\infty .
\end{aligned}
$$

Set

$$
S_{0}=0, \quad S_{n}=\sum_{j=1}^{n} X_{j}, \quad S_{n}^{*}=\max _{1 \leq j \leq n}\left\|S_{j}\right\| \quad \forall n \in \mathbb{N}^{*},
$$

and set

$$
X_{0}^{*}=0, \quad X_{n}^{*}=\max _{1 \leq j \leq n}\left\|X_{j}\right\| \quad \forall n \in \mathbb{N}^{*} .
$$

For $\gamma>0$, let

$$
m(\gamma, n)=\sum_{j=1}^{n} \mathbb{E}\left[\left\|X_{j}\right\|^{\gamma} \mid \mathscr{F}_{j-1}\right] .
$$

Accordingly, for an infinite $\mathbb{B}$-valued adapted sequence $\left\{\left(X_{j}, \mathscr{F}_{j}\right)\right\}_{j \geq 1}$, we write

$$
X_{\infty}^{*}=\sup _{j \geq 1}\left\|X_{j}\right\|, \quad S_{\infty}^{*}=\sup _{j \geq 1}\left\|S_{j}\right\|, \quad S_{\infty}=\sum_{j=1}^{\infty} X_{j}
$$

if the series converges, and

$$
m(\gamma, \infty)=\sum_{j=1}^{\infty} \mathbb{E}\left[\left\|X_{j}\right\|^{\gamma} \mid \mathscr{F}_{j-1}\right] .
$$

In the following, we consider relations among $\sum_{j=1}^{n} \mathbb{P}\left\{\left\|X_{j}\right\|>\varepsilon\right\}, \mathbb{P}\left\{X_{n}^{*}>\varepsilon\right\}, \mathbb{P}\left\{S_{n}^{*}>\varepsilon\right\}$, and $\mathbb{P}\left\{\left\|S_{n}\right\|>\varepsilon\right\}$.

Our first theorem describes relations between $\mathbb{P}\left\{X_{n}^{*}>\varepsilon\right\}$ and $\sum_{j=1}^{n} \mathbb{P}\left\{\left\|X_{j}\right\|>\varepsilon\right\}$ for an adapted sequence of $\mathbb{B}$-valued random variables $\left\{\left(X_{j}, \mathscr{F}_{j}\right)\right\}_{j=1}^{n}$.

Theorem 1. Let $\left\{\left(X_{j}, \mathscr{F}_{j}\right)\right\}_{j=1}^{n}$ be an adapted sequence of $\mathbb{B}$ valued random variables. Then, for any $\varepsilon, \gamma>0$, and $q \geq 1$,

$$
\begin{aligned}
\mathbb{P}\left\{X_{n}^{*}>\varepsilon\right\} & \leq \sum_{j=1}^{n} \mathbb{P}\left\{\left\|X_{j}\right\|>\varepsilon\right\} \\
& \leq\left(1+\varepsilon^{-\gamma}\right) \mathbb{P}\left\{X_{n}^{*}>\varepsilon\right\}+\varepsilon^{-\gamma} \mathbb{E} m^{q}(\gamma, n) .
\end{aligned}
$$

Our second theorem shows relations between $\mathbb{P}\left\{S_{n}^{*}>\right.$ $\varepsilon\}$ and $\mathbb{P}\left\{X_{n}^{*}>\varepsilon\right\}$ for a sequence of $\mathbb{B}$-valued martingale differences $\left\{\left(X_{j}, \mathscr{F}_{j}\right)\right\}_{j=1}^{n}$ : that is, for each (integer) $1 \leq j \leq n$, $X_{j}$ is $\mathscr{F}_{j}$ measurable and belongs to $\mathbb{L}_{\mathbb{B}}^{1}$, and $\mathbb{E}\left[X_{j} \mid \mathscr{F}_{j-1}\right]=0$ a.s.

Theorem 2. Let $\left\{\left(X_{j}, \mathscr{F}_{j}\right)\right\}_{j=1}^{n}$ be a finite sequence of $\mathbb{B}$-valued martingale differences. For any $\varepsilon>0, \gamma \in(1,2], q \geq 1$, and $L \in \mathbb{N}$, if $\mathbb{B}$ is $\gamma$-smooth, then

$$
\begin{aligned}
\mathbb{P}\left\{X_{n}^{*}>2 \varepsilon\right\} \leq & \mathbb{P}\left\{S_{n}^{*}>\varepsilon\right\} \\
\leq & \mathbb{P}\left\{X_{n}^{*}>\frac{\varepsilon}{4(L+1)}\right\} \\
& +\varepsilon^{-q \gamma(1+L) /(q+L)} C(\gamma, q, L) \\
& \times\left(\mathbb{E} m^{q}(\gamma, n)\right)^{(1+L) /(q+L)},
\end{aligned}
$$

where $C(\gamma, q, L)$ is a constant only depending on $\gamma, q$, and $L$.

Corollary 3. Let $\left\{\left(X_{j}, \mathscr{F}_{j}\right)\right\}_{j \geq 1}$ be a sequence of $\mathbb{B}$-valued martingale differences. Suppose that, for some $\gamma \in(1,2]$,

$$
\sum_{j=1}^{\infty} \mathbb{E}\left\|X_{j}\right\|^{\gamma}<\infty
$$

If $\mathbb{B}$ is $\gamma$-smooth, then $S_{\infty}$ converges a.s. and the inequalities (14) and (15) hold with $n$ replaced by $\infty$.

We get Theorems 1 and 2 by a refinement of the method of Alsmeyer [18].

Proof of Theorem 1. The first inequality is obvious. We only consider the second one. Clearly,

$$
\begin{aligned}
\mathbb{P}\left\{X_{n}^{*}>\varepsilon\right\} & =\sum_{j=1}^{n} \mathbb{P}\left\{X_{j-1}^{*} \leq \varepsilon,\left\|X_{j}\right\|>\varepsilon\right\} \\
& =\sum_{j=1}^{n} \mathbb{P}\left\{\left\|X_{j}\right\|>\varepsilon\right\}-\sum_{j=2}^{n} \mathbb{P}\left\{X_{j-1}^{*}>\varepsilon,\left\|X_{j}\right\|>\varepsilon\right\} .
\end{aligned}
$$


Since $\left\{\left(X_{j}, \mathscr{F}_{j}\right)\right\}_{j=1}^{n}$ is an adapted sequence of $\mathbb{B}$-valued random variables, by Markov's inequality (conditional on $\left.\mathscr{F}_{j-1}\right)$,

$$
\begin{aligned}
\mathbb{P}\left\{X_{j-1}^{*}>\varepsilon,\left\|X_{j}\right\|>\varepsilon\right\} & =\int_{\left\{X_{j-1}^{*}>\varepsilon\right\}} \mathbf{1}_{\left\{\left\|X_{j}\right\|>\varepsilon\right\}} \mathrm{d} \mathbb{P} \\
& =\int_{\left\{X_{j-1}^{*}>\varepsilon\right\}} \mathbb{P}\left\{\left\|X_{j}\right\|>\varepsilon \mid \mathscr{F}_{j-1}\right\} \mathrm{d} \mathbb{P} \\
& \leq \varepsilon^{-\gamma} \int_{\left\{X_{j-1}^{*}>\varepsilon\right\}} \mathbb{E}\left[\left\|X_{j}\right\|^{\gamma} \mid \mathscr{F}_{j-1}\right] \mathrm{d} \mathbb{P} \\
& \leq \varepsilon^{-\gamma} \int_{\left\{X_{n}^{*}>\varepsilon\right\}} \mathbb{E}\left[\left\|X_{j}\right\|^{\gamma} \mid \mathscr{F}_{j-1}\right] \mathrm{d} \mathbb{P} .
\end{aligned}
$$

Hence, by summing, we obtain

$$
\begin{aligned}
\sum_{j=2}^{n} \mathbb{P} & \left\{X_{j-1}^{*}>\varepsilon,\left\|X_{j}\right\|>\varepsilon\right\} \\
\leq & \varepsilon^{-\gamma} \int_{\left\{X_{n}^{*}>\varepsilon\right\}} m(\gamma, n) \mathrm{d} \mathbb{P} \\
= & \varepsilon^{-\gamma} \int_{\left\{m(\gamma, n) \leq 1, X_{n}^{*}>\varepsilon\right\}} m(\gamma, n) \mathrm{d} \mathbb{P} \\
& +\varepsilon^{-\gamma} \int_{\left\{m(\gamma, n)>1, X_{n}^{*}>\varepsilon\right\}} m(\gamma, n) \mathrm{d} \mathbb{P} \\
\leq & \varepsilon^{-\gamma} \mathbb{P}\left\{X_{n}^{*}>\varepsilon\right\}+\varepsilon^{-\gamma} \int_{\{m(\gamma, n)>1\}} m^{q}(\gamma, n) \mathrm{d} \mathbb{P} \\
\leq & \varepsilon^{-\gamma} \mathbb{P}\left\{X_{n}^{*}>\varepsilon\right\}+\varepsilon^{-\gamma} \mathbb{E} m^{q}(\gamma, n) .
\end{aligned}
$$

Therefore, the upper bound in (19) gives a lower bound of $\mathbb{P}\left\{X_{n}^{*}>\varepsilon\right\}$ by (17), which implies the second inequality of (14).

Proof of Theorem 2. The first inequality is obvious, because if $\max _{1 \leq j \leq n}\left\|S_{j}\right\| \leq \varepsilon$, then

$$
\max _{1 \leq j \leq n}\left\|X_{j}\right\|=\max _{1 \leq j \leq n}\left\|S_{j}-S_{j-1}\right\| \leq 2 \varepsilon .
$$

We will prove the second inequality. For any $\varepsilon>0, n \in \mathbb{N}^{*}$, and $L \in \mathbb{N}$,

$$
\begin{aligned}
& \mathbb{P}\left\{S_{n}^{*}>2 \varepsilon\right\} \\
& \quad \leq \mathbb{P}\left\{X_{n}^{*}>\frac{\varepsilon}{2(L+1)}\right\}+\mathbb{P}\left\{S_{n}^{*}>2 \varepsilon, X_{n}^{*} \leq \frac{\varepsilon}{2(L+1)}\right\} .
\end{aligned}
$$

Define

$$
\begin{gathered}
T(0)=0, \\
T(j)=\inf \left\{i \in(T(j-1), n]:\left\|S_{i}-S_{T(j-1)}\right\|>\frac{\varepsilon}{L+1}\right\} \\
\text { for } 1 \leq j \leq L+1,
\end{gathered}
$$

where by convention inf $\emptyset=+\infty$. It is easily seen that $T(j)$ are stopping times (cf. e.g., [34] for the definition) with respect to the filtration $\mathscr{F}_{0}=\{\emptyset, \Omega\} \subset \mathscr{F}_{1} \subset \cdots \subset \mathscr{F}_{n} \subset \mathscr{F}_{n+1} \subset \cdots$, where we take $\mathscr{F}_{k}=\mathscr{F}_{n}$ for all $k \geq n$. As usual, we will write $\mathscr{F}_{T(j)}=\left\{A \in \mathscr{F}_{n}: A \cap\{T(j)=k\} \in \mathscr{F}_{k}, 1 \leq k \leq n\right\}$. Notice that for $1 \leq j \leq L+1$, if $T(j)<\infty$, then $\| S_{T(j)}-$ $S_{T(j-1)} \|>\varepsilon /(L+1)$; conversely, if there exists a positive integer $i \in(T(j-1), n]$ such that $\left\|S_{i}-S_{T(j-1)}\right\|>\varepsilon /(L+1)$, then $T(j)<\infty$. We proceed by three steps to estimate the second term of the right hand side of (21).

(a) We first prove that

$$
\left\{S_{n}^{*}>2 \varepsilon, X_{n}^{*} \leq \frac{\varepsilon}{2(L+1)}\right\} \subset \prod_{j=1}^{L+1}\{T(j)<\infty\},
$$

which implies that

$$
\begin{aligned}
& \mathbb{P}\left\{S_{n}^{*}>2 \varepsilon, X_{n}^{*} \leq \frac{\varepsilon}{2(L+1)}\right\} \\
& \leq \mathbb{P}\left\{V_{j}>\frac{\varepsilon}{L+1}, 1 \leq j \leq L+1\right\},
\end{aligned}
$$

where

$$
V_{j}=\left\|S_{T(j)}-S_{T(j-1)}\right\| \mathbf{1}_{\{T(j)<\infty\}}, \quad 1 \leq j \leq L+1 .
$$

Assume that the first event in (23) takes place. Since $S_{n}^{*}>2 \varepsilon$, there exists $m \in\{1,2, \ldots, n\}$ such that $\left\|S_{m}\right\|>2 \varepsilon$. As $\left\|S_{m}\right\|>$ $2 \varepsilon$, and it is clear that $T(1) \leq m<\infty$.

Let $M$ be the largest $j \in[1, m]$ such that $T(j) \leq m$. Then, $T(M) \leq m$.

We will prove that $M \geq L+1$. Suppose that $M \leq L$. Then, by the definition of $M, T(M+1)>m$ so that

$$
\left\|S_{m}-S_{T(M)}\right\| \leq \frac{\varepsilon}{L+1}
$$

As $\|x+y\| \leq\|x\|+\|y\|$, it follows that

$$
\begin{aligned}
\left\|S_{T(j)}-S_{T(j-1)}\right\| & =\left\|S_{T(j)-1}-S_{T(j-1)}+X_{T(j)}\right\| \\
& \leq\left\|S_{T(j)-1}-S_{T(j-1)}\right\|+\left\|X_{T(j)}\right\| \\
& \leq \frac{\varepsilon}{L+1}+\frac{\varepsilon}{2(L+1)},
\end{aligned}
$$

where the last step holds because $\left\|S_{T(j)-1}-S_{T(j-1)}\right\| \leq \varepsilon /(L+1)$ by the definition of $T(j)$ and $\left\|X_{T(j)}\right\| \leq X_{n}^{*} \leq \varepsilon / 2(L+1)$. As $S_{m}=\sum_{j=1}^{M}\left(S_{T(j)}-S_{T(j-1)}\right)+\left(S_{m}-S_{T(M)}\right)$, by (26), (27), and the subadditivity of $\|\cdot\|$, we know that

$$
\left\|S_{m}\right\| \leq \frac{\varepsilon}{L+1} \cdot M+\frac{\varepsilon}{2(L+1)} \cdot M+\frac{\varepsilon}{L+1}<2 \varepsilon
$$

This is a contradiction with $\left\|S_{m}\right\|>2 \varepsilon$, which proves that $M \geq$ $L+1$. 
Therefore, $T(j)<\infty$ for all $j \in[1, L+1]$. Thus, (23) holds.

(b) We next give an estimation of $\mathbb{E}\left[V_{j}^{\gamma} \mid \mathscr{F}_{T(j-1)}\right]$. For $0 \leq r \leq n$,

$$
\begin{aligned}
\mathbb{E} & {\left[V_{j}^{\gamma} \mathbf{1}_{\{T(j-1)=r\}} \mid \mathscr{F}_{T(j-1)}\right] } \\
& =\mathbb{E}\left[V_{j}^{\gamma} \mathbf{1}_{\{T(j-1)=r\}} \mid \mathscr{F}_{r}\right] \\
& \leq \mathbb{E}\left[\left\|S_{T(j)}-S_{T(j-1)}\right\| \mathbf{1}_{\{T(j-1)=r, T(j)<\infty\}} \mid \mathscr{F}_{r}\right] \\
& \leq \mathbf{1}_{\{T(j-1)=r\}} \mathbb{E}\left[\max _{r+1 \leq i \leq n}\left\|S_{i}-S_{r}\right\|^{\gamma} \mid \mathscr{F}_{r}\right] .
\end{aligned}
$$

Using successively Doob's inequality in a real separable Banach space [37, Theorem 3.1], the inequality in Assouad [38, Proposition 2] (conditional on $\mathscr{F}_{r}$ ), and the subadditivity of the function $x \mapsto x^{\gamma / 2} \quad(x \geq 0)$, we obtain

$$
\begin{aligned}
& \mathbb{E}\left[\max _{r+1 \leq i \leq n}\left\|S_{i}-S_{r}\right\|^{\gamma} \mid \mathscr{F}_{r}\right] \\
& \leq\left(\frac{\gamma}{\gamma-1}\right)^{\gamma} \mathbb{E}\left[\left\|\sum_{i=r+1}^{n} X_{i}\right\|^{\gamma} \mid \mathscr{F}_{r}\right] \\
& \leq\left(\frac{\gamma}{\gamma-1}\right)^{\gamma} C(\gamma) \sum_{i=r+1}^{n} \mathbb{E}\left[\left\|X_{i}\right\|^{\gamma} \mid \mathscr{F}_{r}\right] \\
& =\left(\frac{\gamma}{\gamma-1}\right)^{\gamma} C(\gamma) \mathbb{E}\left[\sum_{i=r+1}^{n} \mathbb{E}\left[\left\|X_{i}\right\|^{\gamma} \mid \mathscr{F}_{i-1}\right] \mid \mathscr{F}_{r}\right] \\
& \leq\left(\frac{\gamma}{\gamma-1}\right)^{\gamma} C(\gamma) \mathbb{E}\left[m(\gamma, n) \mid \mathscr{F}_{r}\right] .
\end{aligned}
$$

Therefore, by (29),

$$
\begin{aligned}
\mathbb{E} & {\left[V_{j}^{\gamma} \mathbf{1}_{\{T(j-1)=r\}} \mid \mathscr{F}_{T(j-1)}\right] } \\
\leq & \left(\frac{\gamma}{\gamma-1}\right)^{\gamma} C(\gamma) \mathbb{E}\left[m(\gamma, n) \mathbf{1}_{\{T(j-1)=r\}} \mid \mathscr{F}_{T(j-1)}\right] .
\end{aligned}
$$

Summing over $r$, we obtain

$$
\mathbb{E}\left[V_{j}^{\gamma} \mid \mathscr{F}_{T(j-1)}\right] \leq\left(\frac{\gamma}{\gamma-1}\right)^{\gamma} C(\gamma) \mathbb{E}\left[m(\gamma, n) \mid \mathscr{F}_{T(j-1)}\right] .
$$

(c) We finally give un upper bound for the term of the right hand side of (24), using (32). Set

$$
Q_{j}=\mathbb{P}\left\{V_{j}>\frac{\varepsilon}{L+1} \mid \mathscr{F}_{T(j-1)}\right\}, \quad 1 \leq j \leq L+1 .
$$

Applying (32) for $j=1$, we see that

$$
\begin{aligned}
Q_{1} & =\mathbb{P}\left\{V_{1}>\frac{\varepsilon}{L+1}\right\} \leq \varepsilon^{-\gamma}(L+1)^{\gamma} \mathbb{E} V_{1}^{\gamma} \\
& \leq \varepsilon^{-\gamma}(L+1)^{\gamma}\left(\frac{\gamma}{\gamma-1}\right)^{\gamma} C(\gamma) \mathbb{E} m(\gamma, n) .
\end{aligned}
$$

Now, for any $x>0$,

$$
\mathbb{P}\left\{V_{j}>\frac{\varepsilon}{L+1}, 1 \leq j \leq L+1\right\} \leq \sum_{i=1}^{L+1} p_{i},
$$

where

$$
\begin{gathered}
p_{1}=\mathbb{P}\left\{V_{j}>\frac{\varepsilon}{L+1}, 1 \leq j \leq L+1 ; Q_{i} \leq x^{-1}, 2 \leq i \leq L+1\right\}, \\
p_{i}=\mathbb{P}\left\{Q_{i}>x^{-1}\right\}, \quad 2 \leq i \leq L+1 .
\end{gathered}
$$

Notice that

$$
\begin{aligned}
p_{1} & =\int_{\substack{\left\{V_{j}>\varepsilon /(L+1), 1 \leq j \leq L ; \\
Q_{i} \leq x^{-1}, 2 \leq i \leq L+1\right\}}} \mathbb{E}\left[\mathbf{1}_{\left\{V_{L+1}>\varepsilon /(L+1)\right\}} \mid \mathscr{F}_{T(L)}\right] \mathrm{d} \mathbb{P} \\
& =\int_{\left\{V_{j}>\varepsilon /(L+1), 1 \leq j \leq L ; Q_{i} \leq x^{-1}, 2 \leq i \leq L+1\right\}} Q_{L+1} \mathrm{dPP} \\
& \leq x^{-1} \mathbb{P}\left\{V_{j}>\frac{\varepsilon}{L+1}, 1 \leq j \leq L ; Q_{i} \leq x^{-1}, 2 \leq i \leq L\right\} \\
& \leq \cdots \leq x^{-(L-1)} \mathbb{P}\left\{V_{j}>\frac{\varepsilon}{L+1}, 1 \leq j \leq 2 ; Q_{2} \leq x^{-1}\right\} \\
& \leq x^{-L} Q_{1} \leq \varepsilon^{-\gamma}(L+1)^{\gamma}\left(\frac{\gamma}{\gamma-1}\right)^{\gamma} C(\gamma) x^{-L} \mathbb{E} m(\gamma, n),
\end{aligned}
$$

where (34) has been used for the last inequality. For $2 \leq i \leq$ $L+1$, by (32), together with Markov's inequality and Jensen's inequality, we have

$$
\begin{aligned}
p_{i}= & \mathbb{P}\left\{\mathbb{P}\left\{V_{i}>\frac{\varepsilon}{L+1} \mid \mathscr{F}_{T(i-1)}\right\}>x^{-1}\right\} \\
\leq & \mathbb{P}\left\{\mathbb{E}\left[V_{i}^{\gamma} \mid \mathscr{F}_{T(i-1)}\right]>\frac{\mathcal{\varepsilon}^{\gamma} x^{-1}}{(L+1)^{\gamma}}\right\} \\
\leq & \mathbb{P}\left\{\gamma^{\gamma}(\gamma-1)^{-\gamma} C(\gamma) \mathbb{E}\left[m(\gamma, n) \mid \mathscr{F}_{T(i-1)}\right]>\frac{\varepsilon^{\gamma} x^{-1}}{(L+1)^{\gamma}}\right\} \\
\leq & \varepsilon^{-q \gamma}(L+1)^{q \gamma}\left(\frac{\gamma}{\gamma-1}\right)^{q \gamma} C^{q}(\gamma) \\
& \times x^{q} \mathbb{E}\left(\mathbb{E}\left[m(\gamma, n) \mid \mathscr{F}_{T(i-1)}\right]\right)^{q} \\
\leq & \varepsilon^{-q \gamma}(L+1)^{q \gamma}\left(\frac{\gamma}{\gamma-1}\right)^{q \gamma} C^{q}(\gamma) x^{q} \mathbb{E} m^{q}(\gamma, n) .
\end{aligned}
$$

Therefore, by (35),

$$
\mathbb{P}\left\{V_{j}>\frac{\varepsilon}{L+1}, 1 \leq j \leq L+1\right\} \leq C_{1} x^{-L}+C_{2} x^{q},
$$

where

$$
\begin{aligned}
& C_{1}=\varepsilon^{-\gamma}(L+1)^{\gamma}\left(\frac{\gamma}{\gamma-1}\right)^{\gamma} C(\gamma) \mathbb{E} m(\gamma, n), \\
& C_{2}=\varepsilon^{-q \gamma} L(L+1)^{q \gamma}\left(\frac{\gamma}{\gamma-1}\right)^{q \gamma} C^{q}(\gamma) \mathbb{E} m^{q}(\gamma, n) .
\end{aligned}
$$


A simple calculation shows that

$$
\begin{aligned}
\inf _{x>0}\{ & \left.C_{1} x^{-L}+C_{2} x^{q}\right\} \\
= & \varepsilon^{-q \gamma(1+L) /(q+L)} C_{0}(\gamma, q, L)(\mathbb{E} m(\gamma, n))^{q /(q+L)} \\
& \times\left(\mathbb{E} m^{q}(\gamma, n)\right)^{L /(q+L)},
\end{aligned}
$$

where $C_{0}(\gamma, q, L)=(L+1)^{q \gamma(1+L) /(q+L)}\left[q^{L /(q+L)}+\right.$ $\left.L q^{-q /(q+L)}\right]\left(\gamma^{\gamma}(\gamma-1)^{-\gamma} C(\gamma)\right)^{q(1+L) /(q+L)}$. Therefore,

$$
\begin{aligned}
\mathbb{P}\{ & \left.V_{j}>\frac{\varepsilon}{1+L}, 1 \leq j \leq L+1\right\} \\
\leq & \varepsilon^{-q \gamma(1+L) /(q+L)} C_{0}(\gamma, q, L)(\mathbb{E} m(\gamma, n))^{q /(q+L)} \\
& \times\left(\mathbb{E} m^{q}(\gamma, n)\right)^{L /(q+L)} \\
\leq & \varepsilon^{-q \gamma(1+L) /(q+L)} C_{0}(\gamma, q, L)\left(\mathbb{E} m^{q}(\gamma, n)\right)^{(1+L) /(q+L)} .
\end{aligned}
$$

Together with (21) and (24), this proves (15).

Proof of Corollary 3. By Theorem 2 with $q=1$ and $L=0$,

$$
\begin{aligned}
& \mathbb{P}\left\{\sup _{j \geq 1}\left\|S_{n+j}-S_{n}\right\|>\varepsilon\right\} \\
& \leq \mathbb{P}\left\{\sup _{j \geq n+1}\left\|X_{j}\right\|>\frac{\varepsilon}{4}\right\}+\varepsilon^{-\gamma} C(\gamma) \sum_{j=n+1}^{\infty} \mathbb{E}\left\|X_{j}\right\|^{\gamma} .
\end{aligned}
$$

By Theorem 1 and Markov's inequality,

$$
\begin{aligned}
& \mathbb{P}\left\{\sup _{j \geq n+1}\left\|X_{j}\right\|>\frac{\varepsilon}{4}\right\} \\
& \leq \sum_{j=n+1}^{\infty} \mathbb{P}\left\{\left\|X_{j}\right\|>\frac{\varepsilon}{4}\right\} \leq 4^{\gamma} \varepsilon^{-\gamma} \sum_{j=n+1}^{\infty} \mathbb{E}\left\|X_{j}\right\|^{\gamma} .
\end{aligned}
$$

Therefore,

$$
\lim _{n \rightarrow \infty} \mathbb{P}\left\{\sup _{j \geq 1}\left\|S_{n+j}-S_{n}\right\|>\varepsilon\right\}=0
$$

which is equivalent to

$$
\lim _{n \rightarrow \infty} \sup _{j, k \geq n}\left\|S_{k}-S_{j}\right\|=0 \quad \text { in probability. }
$$

Since $\sup _{j, k \geq n}\left\|S_{k}-S_{j}\right\|$ is decreasing in $n$, this implies that

$$
\lim _{n \rightarrow \infty} \sup _{j, k \geq n}\left\|S_{k}-S_{j}\right\|=0 \quad \text { a.s. }
$$

which gives the desired conclusion.

\section{Convergence Rates for Arrays of Banach Valued Martingale Differences}

In this section, we consider the convergence rates in the law of large numbers for arrays of Banach valued martingale differences.

Let $(\Omega, \mathscr{F}, \mathbb{P})$ a probability space and $(\mathbb{B},\|\cdot\|)$ be a real separable Banach space. For every $n \geq 1$, let $\mathscr{F}_{n 0}=\{\emptyset, \Omega\} \subset$ $\mathscr{F}_{n 1} \subset \cdots$ be an increasing sequence of sub- $\sigma$-fields of $\mathscr{F}$. For every $n \geq 1$, let $\left\{\left(X_{n j}, \mathscr{F}_{n j}\right)\right\}_{j \geq 1}$ be a sequence of $\mathbb{B}$-valued martingale differences defined on $(\Omega, \mathscr{F}, \mathbb{P})$, adapted to the filtration $\left(\mathscr{F}_{n j}\right)$ : that is, for every $j \geq 1, X_{n j}$ is $\mathscr{F}_{n j}$ measurable and belongs to $\mathbb{L}_{\mathbb{B}}^{1}$, and $\mathbb{E}\left[X_{n j} \mid \mathscr{F}_{n, j-1}\right]=0$ a.s. Set for $n \geq 1$,

$$
\begin{aligned}
S_{n 0}=0, \quad S_{n k} & =\sum_{j=1}^{k} X_{n j} \quad \text { for } k \geq 1, \\
S_{n, \infty} & =\sum_{j=1}^{\infty} X_{n j}
\end{aligned}
$$

if the series converges. We will call the double sequence $\left\{\left(X_{n j}, \mathscr{F}_{n j}\right), j \geq 1, n \geq 1\right\}$ an array of $\mathbb{B}$-valued martingale differences.

In the following, we give a sufficient condition for the convergence of $\mathbb{B}$-valued martingale arrays. For $\gamma>0$, let

$$
m_{n}(\gamma)=\sum_{j=1}^{\infty} \mathbb{E}\left[\left\|X_{n j}\right\|^{\gamma} \mid \mathscr{F}_{n, j-1}\right] \text { for } n \geq 1 \text {. }
$$

Theorem 4. Assume that for some $\gamma \in(1,2]$, as $n \rightarrow \infty$,

$$
\mathbb{E} m_{n}(\gamma)=\sum_{j=1}^{\infty} \mathbb{E}\left\|X_{n j}\right\|^{\gamma} \longrightarrow 0 .
$$

If $\mathbb{B}$ is $\gamma$-smooth, then for all $\varepsilon>0$, as $n \rightarrow \infty$,

$$
\begin{aligned}
& \sum_{j=1}^{\infty} \mathbb{P}\left\{\left\|X_{n j}\right\|>\varepsilon\right\} \longrightarrow 0 ; \\
& \mathbb{P}\left\{\sup _{j \geq 1}\left\|X_{n j}\right\|>\varepsilon\right\} \longrightarrow 0 ; \\
& \mathbb{P}\left\{\sup _{j \geq 1}\left\|S_{n j}\right\|>\varepsilon\right\} \longrightarrow 0 ; \\
& \mathbb{P}\left\{\left\|S_{n, \infty}\right\|>\varepsilon\right\} \longrightarrow 0 .
\end{aligned}
$$

Proof. Notice that, by Corollary 3, the condition (50) implies the a.s. convergence of $S_{n, \infty}$. Equation (51) comes from (50) as

$$
\sum_{j=1}^{\infty} \mathbb{P}\left\{\left\|X_{n j}\right\|>\varepsilon\right\} \leq \varepsilon^{-\gamma} \sum_{j=1}^{\infty} \mathbb{E}\left\|X_{n j}\right\|^{\gamma} \longrightarrow 0
$$

(52) follows from (51) and Theorem 1; (53) is a consequence of (52) and Theorem 2; (54) is implied by (53) and Corollary 3. 
We are interested in the convergence rates of the probabilities $\mathbb{P}\left\{\sup _{j \geq 1}\left\|S_{n j}\right\|>\varepsilon\right\}$ and $\mathbb{P}\left\{\left\|S_{n, \infty}\right\|>\varepsilon\right\}$. We will describe their rates of convergence by comparing them with an auxilary function $\phi(n)$ and by considering the convergence of the related series.

We begin with some relations among $\sum_{j=1}^{\infty} \mathbb{P}\left\{\left\|X_{n j}\right\|>\varepsilon\right\}$, $\mathbb{P}\left\{\sup _{j \geq 1}\left\|X_{n j}\right\|>\varepsilon\right\}, \mathbb{P}\left\{\sup _{j \geq 1}\left\|S_{n j}\right\|>\varepsilon\right\}$, and $\mathbb{P}\left\{\left\|S_{n, \infty}\right\|>\varepsilon\right\}$.

Lemma 5. Let $\phi: \mathbb{N} \rightarrow[0, \infty)$ be a positive function. Suppose that for some $\gamma \in(1,2], q \geq 1$ and some integer $L \geq 0$,

$$
\phi(n)\left(\mathbb{E} m_{n}^{q}(\gamma)\right)^{(1+L) /(q+L)}=o(1) \quad(\text { resp., } O(1)) .
$$

If $\mathbb{B}$ is $\gamma$-smooth, then the following conditions are equivalent:

$$
\begin{array}{r}
\phi(n) \mathbb{P}\left\{\sup _{j \geq 1}\left\|X_{n j}\right\|>\varepsilon\right\}=o(1) \\
(\text { resp., } O(1)) \text { for any } \varepsilon>0 ; \\
\phi(n) \mathbb{P}\left\{\sup _{j \geq 1}\left\|S_{n j}\right\|>\varepsilon\right\}=o(1) \\
(\text { resp., } O(1)) \text { for any } \varepsilon>0 .
\end{array}
$$

Proof. Equation (57) are equivalent by Theorem 2.

Lemma 6. Let $\phi: \mathbb{N} \rightarrow[0, \infty)$ be a positive function. Suppose that for some $\gamma \in(1,2]$ and $q \in[1, \infty)$,

$$
\phi(n) \mathbb{E} m_{n}^{q}(\gamma)=o(1) \quad(\text { resp., } O(1)) .
$$

Then, the following conditions are equivalent:

$$
\begin{array}{r}
\phi(n) \mathbb{P}\left\{\sup _{j \geq 1}\left\|X_{n j}\right\|>\varepsilon\right\}=o(1) \\
(\text { resp., } O(1)) \text { for any } \varepsilon>0 ; \\
\phi(n) \sum_{j=1}^{\infty} \mathbb{P}\left\{\left\|X_{n j}\right\|>\varepsilon\right\}=o(1) \\
(\text { resp., } O(1)) \text { for any } \varepsilon>0 .
\end{array}
$$

Proof. The conclusion comes directly from Theorem 1.

Lemma 7. Let $\phi: \mathbb{N} \rightarrow[0, \infty)$ be a positive function. Suppose that (58) holds for some $\gamma \in(1,2]$ and $q \in[1, \infty)$. Then,

$$
\phi(n) \mathbb{P}\left\{\left\|S_{n, \infty}\right\|>\varepsilon\right\}=o(1) \quad(\text { resp., } O(1)) \text { for any } \varepsilon>0
$$

is implied by

$$
\begin{gathered}
\phi(n) \mathbb{P}\left\{\sup _{j \geq 1}\left\|S_{n j}\right\|>\varepsilon\right\}=o(1) \\
(\text { resp., } O(1)) \text { for any } \varepsilon>0 .
\end{gathered}
$$

Proof. The equivalence is an immediate consequence of Corollary 3.

Theorem 8. Let $\phi: \mathbb{N} \rightarrow[0, \infty)$ be a positive function. Suppose that for some $\gamma \in(1,2], q \in[1, \infty]$ and $\lambda \in(0, q)$,

$$
\left\|m_{n}(\gamma)\right\|_{\mathbb{L}_{\mathbb{B}}^{q}} \longrightarrow 0, \quad \phi(n)\left\|m_{n}(\gamma)\right\|_{\mathbb{L}_{\mathbb{B}}^{q}}^{\lambda}=O(1) .
$$

If $\mathbb{B}$ is $\gamma$-smooth, then one has the following implications $(63) \Leftrightarrow(64) \Leftrightarrow(65) \Rightarrow(66)$ :

$$
\begin{aligned}
& \phi(n) \sum_{j=1}^{\infty} \mathbb{P}\left\{\left\|X_{n j}\right\|>\varepsilon\right\}=o(1) \\
& \text { (resp., } O(1)) \text { for any } \varepsilon>0 \text {; } \\
& \phi(n) \mathbb{P}\left\{\sup _{j \geq 1}\left\|X_{n j}\right\|>\varepsilon\right\}=o(1) \\
& \text { (resp., } O(1)) \text { for any } \varepsilon>0 \text {; } \\
& \phi(n) \mathbb{P}\left\{\sup _{j \geq 1}\left\|S_{n j}\right\|>\varepsilon\right\}=o(1) \\
& \text { (resp., } O(1) \text { ) for any } \varepsilon>0 \text {; } \\
& \phi(n) \mathbb{P}\left\{\left\|S_{n, \infty}\right\|>\varepsilon\right\}=o(1) \\
& \text { (resp., } O(1)) \text { for any } \varepsilon>0 \text {. }
\end{aligned}
$$

Remark 9. The condition (62) holds if for some $\gamma \in(1,2], r \in$ $\mathbb{R}$ and $\varepsilon_{1}>0$,

$$
\phi(n)=O\left(n^{r}\right), \quad\left\|m_{n}(\gamma)\right\|_{L_{\mathbb{B}}^{\infty}}=O\left(n^{-\varepsilon_{1}}\right) .
$$

Proof of Theorem 8. Notice that when (62) holds for $q=\infty$ and some $\lambda \in(0, \infty)$, then for $q \in(\lambda, \infty)$,

$$
\phi(n)\left\|m_{n}(\gamma)\right\|_{\mathbb{L}_{\mathbb{B}}^{q}}^{\lambda} \leq \phi(n)\left\|m_{n}(\gamma)\right\|_{\mathbb{L}_{\mathbb{B}}^{\infty}}^{\lambda}=O(1) .
$$

Therefore, we can assume that (62) holds for some $q \in[1, \infty)$ and $\lambda \in(0, q)$. Since as $L \rightarrow \infty, q(1+L) /(q+L) \rightarrow q>\lambda$, we can choose an integer $L \geq 0$ large enough such that $q(1+$ $L) /(q+L)>\lambda$. Therefore, the condition (56) holds with $o(1)$. By Lemma 6, (63) and (64) are equivalent; by Lemma 5, (64) and (65) are equivalent; by Lemma 7, (65) implies (66).

Theorem 10. Let $\phi: \mathbb{N} \rightarrow[0, \infty)$ be a positive function. Suppose that for some $\gamma \in(1,2], q \in[1, \infty]$ and $\lambda \in(0, q)$,

$$
\left\|m_{n}(\gamma)\right\|_{\mathbb{L}_{\mathbb{B}}^{q}} \longrightarrow 0, \quad \sum_{n=1}^{\infty} \phi(n)\left\|m_{n}(\gamma)\right\|_{\mathbb{L}_{\mathbb{B}}^{q}}^{\lambda}<\infty .
$$


If $\mathbb{B}$ is $\gamma$-smooth, then one has the following implications $(70) \Leftrightarrow(71) \Leftrightarrow(72) \Rightarrow(73)$ :

$$
\begin{aligned}
& \sum_{n=1}^{\infty} \phi(n) \sum_{j=1}^{\infty} \mathbb{P}\left\{\left\|X_{n j}\right\|>\varepsilon\right\}<\infty \quad \forall \varepsilon>0 ; \\
& \sum_{n=1}^{\infty} \phi(n) \mathbb{P}\left\{\sup _{j \geq 1}\left\|X_{n j}\right\|>\varepsilon\right\}<\infty \quad \forall \varepsilon>0 ; \\
& \sum_{n=1}^{\infty} \phi(n) \mathbb{P}\left\{\sup _{j \geq 1}\left\|S_{n j}\right\|>\varepsilon\right\}<\infty \quad \forall \varepsilon>0 ; \\
& \sum_{n=1}^{\infty} \phi(n) \mathbb{P}\left\{\left\|S_{n, \infty}\right\|>\varepsilon\right\}<\infty \quad \forall \varepsilon>0 .
\end{aligned}
$$

Notice that, by (69), $\mathbb{E} m_{n}(\gamma)<\infty$ for each $n \geq 1$ with $\phi(n)>0$, so that $S_{n, \infty}$ is well defined (cf. Corollary 3). When $\phi(n)=0$, we use the convention that the associated term containing $\phi(n)$ as a factor is defined by 0 .

When $q=\infty, \gamma=2$, and $\left\{\left(X_{n j}, \mathscr{F}_{n j}\right)\right\}_{j \geq 1}$ is a sequence of real-valued martingale differences, the implication " $(70) \Rightarrow(72)$ " reduces to Theorem 2 of Ghosal and Chandra [19]. (Although the condition $\left\|m_{n}(2)\right\|_{\mathbb{L}_{\mathbb{B}}^{\infty}} \rightarrow 0$ does not appear in Theorem 2 of [19], it is implicitly used in its proof.) So, our result improves and completes that of Ghosal and Chandra [19] in the sense that we prove the equivalence between (70) and (72) (not just the implication " $(70) \Rightarrow(72)$ ") under much weaker conditions.

Remark 11. Theorem 10 also holds if $m_{n}(\gamma)$ is replaced by $\sum_{j=1}^{\infty} \mathbb{E}\left[\left\|X_{n j}\right\|^{\gamma} \mid \mathscr{F}_{n, j-M}\right]$ for some $M \geq 1$. In fact, the case $M \geq 2$ can be reduced to the case $M=1$ by considering the subsequences $\left\{\left(X_{n, l M+i}, \mathscr{F}_{n, l M+i}\right)\right\}_{l \geq 0}(1 \leq i \leq$ $M)$ of $\left\{\left(X_{n k}, \mathscr{F}_{n k}\right)\right\}_{k \geq 1}$, which are still sequences of $\mathbb{B}$-valued martingale differences.

Corollary 12. Suppose that (67) holds for some $\gamma \in$ $(1,2], r \in \mathbb{R}$, and $\varepsilon_{1}>0$. Then one has the implications $(70) \Leftrightarrow(71) \Leftrightarrow(72) \Rightarrow(73)$.

Proof of Theorem 10. As in the proof of Theorem 8, we can assume that $q<\infty$. Since as $L \rightarrow \infty, q(1+L) /(q+L) \rightarrow$ $q>\lambda$, we can choose an integer $L \geq 0$ large enough such that $q(1+L) /(q+L)>\lambda$. Let $n_{0}$ be large enough such that $\left\|m_{n}(\gamma)\right\|_{\mathbb{L}_{\mathbb{B}}^{q}} \leq 1$ for all $n \geq n_{0}$. Then,

$$
\begin{aligned}
\sum_{n=n_{0}}^{\infty} \phi(n)\left(\mathbb{E} m_{n}^{q}(\gamma)\right)^{(1+L) /(q+L)} & =\sum_{n=n_{0}}^{\infty} \phi(n)\left\|m_{n}(\gamma)\right\|_{\mathbb{L}_{\mathbb{B}}^{q}}^{q(1+L) /(q+L)} \\
& \leq \sum_{n=n_{0}}^{\infty} \phi(n)\left\|m_{n}(\gamma)\right\|_{\mathbb{E}_{\mathbb{B}}^{q}}^{\lambda}<\infty .
\end{aligned}
$$

By Theorem 1, (70) and (71) are equivalent; by Theorem 2, (71) and (72) are equivalent; since (69) implies $\mathbb{E} m_{n}(\gamma)<\infty$ for each $n \geq 1$ with $\phi(n)>0$, by Corollary 3, (72) implies (73).
Proof of Corollary 12. Choose $\lambda>(r+1) / \varepsilon_{1}$, then by (67), we have

$$
\begin{gathered}
\left\|m_{n}(\gamma)\right\|_{\mathbb{L}_{\mathbb{B}}^{\infty}} \longrightarrow 0, \\
\sum_{n=1}^{\infty} \phi(n)\left\|m_{n}(\gamma)\right\|_{\mathbb{L}_{\mathbb{B}}^{\infty}}^{\lambda}=\sum_{n=1}^{\infty} O\left(n^{r-\varepsilon_{1} \lambda}\right)<\infty .
\end{gathered}
$$

So, the condition (69) holds for $q=\infty$, and the conclusion follows from Theorem 10.

\section{Convergence Rates for Triangular Arrays of Banach Valued Martingale Differences}

In this section, we consider the convergence rates in the law of large numbers for triangular arrays of Banach valued martingale differences.

Let $(\Omega, \mathscr{F}, \mathbb{P})$ be a probability space and $(\mathbb{B},\|\cdot\|)$ a real separable Banach space. For every $n \geq 1$, let $\mathscr{F}_{n 0}=\{\emptyset, \Omega\} \subset$ $\mathscr{F}_{n 1} \subset \cdots \subset \mathscr{F}_{n n}$ be an increasing sequence of sub- $\sigma$-fields of $\mathscr{F}$. For each $n \geq 1$, let $\left\{\left(X_{n j}, \mathscr{F}_{n j}\right)\right\}_{j=1}^{n}$ be a sequence of $\mathbb{B}$ valued martingale differences defined on $(\Omega, \mathscr{F}, \mathbb{P})$, adapted to the filtration $\left(\mathscr{F}_{n j}\right)$ : that is, for every $1 \leq j \leq n$ and every $n \geq 1, X_{n j}$ is $\mathscr{F}_{n j}$ measurable and belongs to $\mathbb{L}_{\mathbb{B}}^{1}$, and $\mathbb{E}\left[X_{n j} \mid\right.$ $\left.\mathscr{F}_{n, j-1}\right]=0$ a.s. Set for $n \geq 1$,

$$
S_{n 0}=0, \quad S_{n k}=\sum_{j=1}^{k} X_{n j}, \quad 1 \leq k \leq n .
$$

We will call the double sequence $\left\{\left(X_{n j}, \mathscr{F}_{n j}\right), 1 \leq j \leq n, n \geq\right.$ $1\}$ a triangular array of $\mathbb{B}$-valued martingale differences. In the following, we first give a sufficient condition for the convergence of triangular arrays of $\mathbb{B}$-valued martingale. For $\alpha \in \mathbb{R}$ and $\gamma>0$, let

$$
m_{n}(\gamma)=n^{-\gamma \alpha} \sum_{j=1}^{n} \mathbb{E}\left[\left\|X_{n j}\right\|^{\gamma} \mid \mathscr{F}_{n, j-1}\right] \text { for } n \geq 1 .
$$

Theorem 13. Let $\alpha \in \mathbb{R}$. Assume that for some $\gamma \in(1,2]$, as $n \rightarrow \infty$,

$$
\mathbb{E} m_{n}(\gamma)=n^{-\gamma \alpha} \sum_{j=1}^{n} \mathbb{E}\left\|X_{n j}\right\|^{\gamma} \longrightarrow 0
$$

If $\mathbb{B}$ is $\gamma$-smooth, then for all $\varepsilon>0$, as $n \rightarrow \infty$,

$$
\begin{gathered}
\sum_{j=1}^{n} \mathbb{P}\left\{\left\|X_{n j}\right\|>\varepsilon n^{\alpha}\right\} \rightarrow 0 ; \\
\mathbb{P}\left\{\max _{1 \leq j \leq n}\left\|X_{n j}\right\|>\varepsilon n^{\alpha}\right\} \longrightarrow 0 ; \\
\mathbb{P}\left\{\max _{1 \leq j \leq n}\left\|S_{n j}\right\|>\varepsilon n^{\alpha}\right\} \longrightarrow 0 ; \\
\mathbb{P}\left\{\left\|S_{n n}\right\|>\varepsilon n^{\alpha}\right\} \longrightarrow 0 .
\end{gathered}
$$


Proof. It suffices to apply Theorem 4 for the array of $\mathbb{B}$-valued martingale differences $\left\{\left(Y_{n j}, \mathscr{G}_{n j}\right), j \geq 1, n \geq 1\right\}$ defined by

$$
\begin{aligned}
& Y_{n j}= \begin{cases}\frac{X_{n j}}{n^{\alpha}} & \text { if } 1 \leq j \leq n, \\
0 & \text { if } j>n,\end{cases} \\
& \mathscr{G}_{n j}= \begin{cases}\mathscr{F}_{n j} & \text { if } 1 \leq j \leq n, \\
\mathscr{F}_{n n} & \text { if } j>n .\end{cases}
\end{aligned}
$$

We are interested in the convergence rates of the probabilities $\mathbb{P}\left\{\max _{1 \leq j \leq n}\left\|S_{n j}\right\|>\varepsilon n^{\alpha}\right\}$ and $\mathbb{P}\left\{\left\|S_{n n}\right\|>\varepsilon n^{\alpha}\right\}$. We will describe their rates of convergence by comparing them with an auxilary function $\phi(n)$ and by considering the convergence of the related series.

We begin with some relations among $\sum_{j=1}^{n} \mathbb{P}\left\{\left\|X_{n j}\right\|>\right.$ $\left.\varepsilon n^{\alpha}\right\}, \mathbb{P}\left\{\max _{1 \leq j \leq n}\left\|X_{n j}\right\|>\varepsilon n^{\alpha}\right\}, \mathbb{P}\left\{\max _{1 \leq j \leq n}\left\|S_{n j}\right\|>\varepsilon n^{\alpha}\right\}$, and $\mathbb{P}\left\{\left\|S_{n n}\right\|>\varepsilon n^{\alpha}\right\}$.

Theorem 14. Let $\alpha \in \mathbb{R}$ and $\phi: \mathbb{N} \rightarrow[0, \infty)$ be a positive function. Suppose that for some $\gamma \in(1,2], q \in[1, \infty]$ and $\lambda \in(0, q)$,

$$
\left\|m_{n}(\gamma)\right\|_{\mathbb{L}_{\mathbb{B}}^{q}} \longrightarrow 0, \quad \phi(n)\left\|m_{n}(\gamma)\right\|_{\mathbb{L}_{\mathbb{B}}^{q}}^{\lambda}=O(1) .
$$

If $\mathbb{B}$ is $\gamma$-smooth, then one has the following implications $(82) \Leftrightarrow(83) \Leftrightarrow(84) \Rightarrow(85)$ :

$$
\begin{array}{r}
\phi(n) \sum_{j=1}^{n} \mathbb{P}\left\{\left\|X_{n j}\right\|>\varepsilon n^{\alpha}\right\}=o(1) \\
(\text { resp., } O(1)) \text { for any } \varepsilon>0 ; \\
\phi(n) \mathbb{P}\left\{\max _{1 \leq j \leq n}\left\|X_{n j}\right\|>\varepsilon n^{\alpha}\right\}=o(1) \\
(\operatorname{resp} ., O(1)) \text { for any } \varepsilon>0 ; \\
\phi(n) \mathbb{P}\left\{\max _{1 \leq j \leq n}\left\|S_{n j}\right\|>\varepsilon n^{\alpha}\right\}=o(1) \\
(\operatorname{resp} ., O(1)) \text { for any } \varepsilon>0 ; \\
\phi(n) \mathbb{P}\left\{\left\|S_{n n}\right\|>\varepsilon n^{\alpha}\right\}=o(1) \\
(\text { resp. }, O(1)) \text { for any } \varepsilon>0 .
\end{array}
$$

Corollary 15. Let $1 / 2<\alpha \leq 1$ and $b \geq 0$. Let $l(\cdot)>0$ be $a$ function slowly varying at $\infty$ and $\phi(n)=n^{b} l(n)$. Suppose that for some $\gamma \in(1 / \alpha, 2], q \in[1, \infty]$ with $q>b /(\gamma \alpha-1)$,

$$
\begin{aligned}
& \sup _{n \geq 1}\left\|\underline{m}_{n}(\gamma)\right\|_{\mathbb{L}_{\mathbb{B}}^{q}}<\infty, \\
& \text { where } \underline{m}_{n}(\gamma)=\frac{1}{n} \sum_{j=1}^{n} \mathbb{E}\left[\left\|X_{n j}\right\|^{\gamma} \mid \mathscr{F}_{n, j-1}\right] .
\end{aligned}
$$

If $\mathbb{B}$ is $\gamma$-smooth, then one has the implications $(82) \Leftrightarrow(83) \Leftrightarrow$ $(84) \Rightarrow(85)$.
Remark 16. It is obvious that (86) holds with $q=\infty$ if for some constant $K>0$, all $n \geq 1$ and $j \geq 1$,

$$
\mathbb{E}\left[\left\|X_{n j}\right\|^{\gamma} \mid \mathscr{F}_{n, j-1}\right] \leq K \quad \text { a.s.. }
$$

Proof of Theorem 14. It suffices to apply Theorem 8 for the array of $\mathbb{B}$-valued martingale differences $\left\{\left(Y_{n j}, \mathscr{G}_{n j}\right), j \geq\right.$ $1, n \geq 1$ \} defined by (80).

Proof of Corollary 15. Since $\gamma>1 / \alpha$, we have

$$
\left\|m_{n}(\gamma)\right\|_{\mathbb{L}_{\mathbb{B}}^{q}}=n^{1-\gamma \alpha}\left\|\underline{m}_{n}(\gamma)\right\|_{\mathbb{L}_{\mathbb{B}}^{q}} \longrightarrow 0 .
$$

As $q>b /(\gamma \alpha-1)$, we can choose $\lambda \in(b /(\gamma \alpha-1), q)$. For this $\lambda$,

$$
\begin{aligned}
\phi(n)\left\|m_{n}(\gamma)\right\|_{\mathbb{L}_{\mathbb{B}}^{q}}^{\lambda} & =n^{b} l(n)\left(n^{1-\gamma \alpha}\left\|\underline{m}_{n}(\gamma)\right\|_{\mathbb{L}_{\mathbb{B}}^{q}}\right)^{\lambda} \\
& =n^{b-\lambda(\gamma \alpha-1)} l(n)\left\|\underline{m}_{n}(\gamma)\right\|_{\mathbb{L}_{\mathbb{B}}^{q}}^{\lambda}=o(1) .
\end{aligned}
$$

Thus, the condition (81) holds, so that the conclusion follows from Theorem 14.

Theorem 17. Let $\alpha \in \mathbb{R}$ and $\phi: \mathbb{N} \rightarrow[0, \infty)$ be a positive function. Suppose that for some $\gamma \in(1,2], q \in[1, \infty]$ and $\lambda \in(0, q)$,

$$
\left\|m_{n}(\gamma)\right\|_{\mathbb{L}_{\mathbb{B}}^{q}} \longrightarrow 0, \quad \sum_{n=1}^{\infty} \phi(n)\left\|m_{n}(\gamma)\right\|_{\mathbb{L}_{\mathbb{B}}^{q}}^{\lambda}<\infty .
$$

If $\mathbb{B}$ is $\gamma$-smooth, then one has the following implications $(91) \Leftrightarrow(92) \Leftrightarrow(93) \Rightarrow(94)$ :

$$
\begin{aligned}
& \sum_{n=1}^{\infty} \phi(n) \sum_{j=1}^{n} \mathbb{P}\left\{\left\|X_{n j}\right\|>\varepsilon n^{\alpha}\right\}<\infty \forall \varepsilon>0 ; \\
& \sum_{n=1}^{\infty} \phi(n) \mathbb{P}\left\{\max _{1 \leq j \leq n}\left\|X_{n j}\right\|>\varepsilon n^{\alpha}\right\}<\infty \quad \forall \varepsilon>0 ; \\
& \sum_{n=1}^{\infty} \phi(n) \mathbb{P}\left\{\max _{1 \leq j \leq n}\left\|S_{n j}\right\|>\varepsilon n^{\alpha}\right\}<\infty \quad \forall \varepsilon>0 ; \\
& \sum_{n=1}^{\infty} \phi(n) \mathbb{P}\left\{\left\|S_{n n}\right\|>\varepsilon n^{\alpha}\right\}<\infty \quad \forall \varepsilon>0 .
\end{aligned}
$$

Corollary 18. Let $\alpha>1 / 2$ and $b \geq 0$. Let $l(\cdot)>0$ be $a$ function slowly varying at $\infty$ and $\phi(n)=n^{b-1} l(n)$. Suppose that (86) holds for some $\gamma \in(\max \{1 / \alpha, 1\}, 2]$ and $q \in[1, \infty]$ with $q>b /(\gamma \alpha-1)$. If $\mathbb{B}$ is $\gamma$-smooth, then one has the implications $(91) \Leftrightarrow(92) \Leftrightarrow(93) \Rightarrow(94)$.

For a single real-valued martingale, when $l(n)=1$ and $\alpha \leq 1$, Corollary 18 reduces to Alsmeyer's result in [18]. We notice that the consideration of a triangular array makes the result very adapted to study weighted sums of identically distributed $\mathbb{B}$-valued random variables of the form $\sum_{j=1}^{n} a_{n j} X_{j}$. 
Remark 19. As explained in Remark 11, Corollary 18 also holds if $\bar{m}_{n}(\gamma)$ is replaced by $(1 / n) \sum_{j=1}^{n} \mathbb{E}\left[\left\|X_{n j}\right\|^{\gamma} \mid \mathscr{F}_{n, j-M}\right]$ for some $M \geq 1$.

Proof of Theorem 17. It suffices to apply Theorem 10 for the array of $\mathbb{B}$-valued martingale differences $\left\{\left(Y_{n j}, \mathscr{G}_{n j}\right), j \geq\right.$ $1, n \geq 1\}$ defined by (80).

Proof of Corollary 18. Notice that

$$
\left\|m_{n}(\gamma)\right\|_{\mathbb{L}_{\mathbb{B}}^{q}}=n^{1-\gamma \alpha}\left\|\bar{m}_{n}(\gamma)\right\|_{\mathbb{L}_{\mathbb{B}}^{q}} \longrightarrow 0 .
$$

As $q>b /(\gamma \alpha-1)$, we can take $\lambda \in(b /(\gamma \alpha-1), q)$.

Then,

$$
\begin{aligned}
\sum_{n=1}^{\infty} \phi(n)\left\|m_{n}(\gamma)\right\|_{\mathbb{E}_{\mathbb{B}}^{q}}^{\lambda} & =\sum_{n=1}^{\infty} n^{b-1} l(n)\left(n^{1-\gamma \alpha}\left\|\bar{m}_{n}(\gamma)\right\|_{\mathbb{L}_{\mathbb{B}}^{q}}\right)^{\lambda} \\
& =\sum_{n=1}^{\infty} n^{b-1-\lambda(\gamma \alpha-1)} l(n)\left\|\bar{m}_{n}(\gamma)\right\|_{\mathbb{E}_{\mathbb{B}}^{q}}^{\lambda}<\infty .
\end{aligned}
$$

Thus, the result holds by Theorem 17 .

As a special case, we obtain the following extension of a result of Chow and Teicher [34, page 393] about the complete convergence on sums of independent random variables.

Corollary 20. Let $\left\{\left(X_{n j}, \mathscr{F}_{n j}\right)\right\}_{1 \leq j \leq n}(n \geq 1)$ be sequences of identically distributed $\mathbb{B}$-valued martingale differences. Let $p \in$ $[1,4)$. Suppose that $(86)$ holds for some $\gamma \in(\max \{p / 2,1\}, 2]$ and $q \in[1, \infty]$ with $q>p /(2 \gamma-p)$. If $\mathbb{B}$ is $\gamma$-smooth, then $\mathbb{E}\left\|X_{11}\right\|^{p}<\infty$ if and only if

$$
\sum_{n=1}^{\infty} \mathbb{P}\left\{n^{-2 / p} \max _{1 \leq j \leq n}\left\|\sum_{i=1}^{j} X_{n i}\right\|>\varepsilon\right\}<\infty \quad \forall \varepsilon>0 .
$$

When $\left\{X_{n j}\right\}$ are rowwise independent real-valued martingale differences, the sufficiency in Corollary 20 was proved in [34, page 393].

Proof of Corollary 20. It suffices to apply Corollary 18 with $\alpha=2 / p$ and $l(n)=1$ : we just need to check that in the present case, (91) is equivalent to $\mathbb{E}\left\|X_{11}\right\|^{p}<\infty$. In fact, we have

$$
\begin{aligned}
\sum_{n=1}^{\infty} \sum_{j=1}^{n} \mathbb{P}\left\{n^{-2 / p}\left\|X_{n j}\right\|>\varepsilon\right\} & =\sum_{n=1}^{\infty} n \mathbb{P}\left\{\left\|X_{11}\right\|>\varepsilon n^{2 / p}\right\} \\
& =\mathbb{E}\left(\sum_{1 \leq n<\varepsilon^{-p / 2}\left\|X_{11}\right\|^{p / 2}} n\right) .
\end{aligned}
$$

As $\sum_{n=1}^{m} n=(1 / 2) m(m+1)$, the last expectation is finite if and only if $\mathbb{E}\left\|X_{11}\right\|^{p}<\infty$.

\section{Convergence Rate for the Maxima of any Banach Valued Random Variables}

In this section, we study the convergence rate for the maxima of a sequence of any Banach valued random variables to obtain further equivalent conditions about the convergence rate for a Banach valued martingale in Section 6.

Let $(\mathbb{B},\|\cdot\|)$ a separable Banach space and $\left(Y_{n}\right)_{n \geq 1}$ be a sequence of any $\mathbb{B}$-valued random variables. For any $v \in$ $[1, \infty)$, let $[v]$ be the integer part of $v$. Set

$$
Y_{0}=0, \quad Y_{v}=Y_{[v]}, \quad Y_{v}^{*}=\max _{0 \leq k \leq v}\left\|Y_{k}\right\|
$$

for $v \in[0, \infty)$.

Then, for any $n \geq 1, Y_{n}^{*}=\max _{0 \leq k \leq n}\left\|Y_{k}\right\|$. For any $\alpha>0, \varepsilon>$ 0 , set

$$
\begin{aligned}
M(\varepsilon) & =M(\varepsilon, \alpha)=\sup _{n \geq 1}\left(\left\|Y_{n}\right\|-\varepsilon n^{\alpha}\right)_{+} \\
& =\sup _{n \geq 1}\left(Y_{n}^{*}-\varepsilon n^{\alpha}\right)_{+}=\sup _{n \geq 0}\left(Y_{n}^{*}-\varepsilon n^{\alpha}\right) .
\end{aligned}
$$

Let $l(\cdot)>0$ be a function slowly varying at $\infty$. Recall that a function $l(x)>0$ slowly varying at $\infty$ has the representation form

$$
l(x)=c(x) \exp \left\{\int_{x_{0}}^{x} \frac{\varepsilon(u)}{u} \mathrm{~d} u\right\} \quad\left(x \geq x_{0}\right)
$$

for some $x_{0}>0$, where $c(\cdot)$ is measurable and $c(x) \rightarrow c \in$ $(0, \infty), \varepsilon(x) \rightarrow 0$ as $x \rightarrow \infty$. The function $c(\cdot)$ plays no role for our purpose. We can choose $c(x) \equiv 1$ without loss of generality.

We are interested in the convergence rates of $\mathbb{P}\left\{Y_{n}^{*}>\varepsilon n^{\alpha}\right\}$ and $\mathbb{P}\left\{\sup _{j \geq n} j^{-\alpha} Y_{j}^{*}>\varepsilon\right\}$. Notice that $\mathbb{P}\left\{\sup _{j \geq n} j^{-\alpha} Y_{j}^{*}>\varepsilon\right\} \rightarrow$ 0 for any $\varepsilon>0$ if and only if $Y_{n}^{*} / n^{\alpha} \rightarrow 0$ a.s. So, our results in this section describe the rate convergence for the almost surely convergence of $Y_{n}^{*} / n^{\alpha}$.

The following result shows that $\mathbb{P}\left\{Y_{n}^{*}>\varepsilon n^{\alpha}\right\}$ and $\mathbb{P}\left\{M(\varepsilon)>\varepsilon n^{\alpha}\right\}$ have similar asymptotic properties. More precise comparisons will be given in Theorems 22 and 24 .

Lemma 21. Let $\alpha>0$. Then, for any $v \geq 1$ and any $\varepsilon>0$,

$$
\mathbb{P}\left\{Y_{v}^{*}>2 \varepsilon v^{\alpha}\right\} \leq \mathbb{P}\left\{\sup _{j \geq v} j^{-\alpha} Y_{j}^{*}>2 \varepsilon\right\} \leq \mathbb{P}\left\{M(\varepsilon)>\varepsilon v^{\alpha}\right\} .
$$

Let $\varepsilon, \alpha, b, \delta>0$ and $\phi(v)=v^{b} l(v)$. If there exists $v_{0}>0$, such that for all $v \geq v_{0}$,

$$
\phi(v) \mathbb{P}\left\{Y_{v}^{*}>\frac{1}{2} \varepsilon v^{\alpha}\right\} \leq \delta
$$

then there exists $v_{0}^{\prime}>0$ depending only on $v_{0}, b$, and $\alpha$, such that for all $v \geq v_{0}^{\prime}$,

$$
\phi(v) \mathbb{P}\left\{M(\varepsilon)>\varepsilon v^{\alpha}\right\} \leq \delta C(b, \alpha),
$$

where $C(b, \alpha)=2 /\left(2^{b /(2 \alpha)}-1\right)$. 
Proof. The first inequality of (102) is obvious. If

$$
\sup _{j \geq v} j^{-\alpha} Y_{j}^{*}>2 \varepsilon
$$

then

$$
\begin{aligned}
\sup _{n \geq 1}\left(Y_{n}^{*}-\varepsilon n^{\alpha}\right)_{+} & =\sup _{n \geq 1}^{\alpha}\left(n^{-\alpha} Y_{n}^{*}-\varepsilon\right)_{+} \\
& \geq v^{\alpha} \sup _{n \geq v}\left(n^{-\alpha} Y_{n}^{*}-\varepsilon\right)_{+}>\varepsilon v^{\alpha} .
\end{aligned}
$$

Thus, the second inequality of (102) holds.

Assume that for some $v_{0}>0$ and all $v \geq v_{0}$, (103) holds (with the notation introduced in the lemma). Then, there exists $v_{2}=v_{2}\left(v_{0}, b, \alpha\right)>0$, such that for all $v_{1} \geq v_{2}$,

$$
\mathbb{P}\left\{Y_{2^{k / \alpha} v_{1}^{1 / b}}^{*} 2^{k-1} \varepsilon v_{1}^{\alpha / b}\right\} \leq \frac{\delta}{\phi\left(2^{k / \alpha} v_{1}^{1 / b}\right)} .
$$

Set $\beta=b / \alpha$. Then, applying (103) for $v=2^{k / \alpha} v_{1}^{1 / b}$, we see that, for any $v_{1} \geq v_{2}$,

$$
\begin{aligned}
& \mathbb{P}\left\{\sup _{n \geq 1}\left(Y_{n}^{*}-\varepsilon n^{\alpha}\right)_{+}>\varepsilon v_{1}^{1 / \beta}\right\} \\
& =\mathbb{P}\left\{\sup _{n \geq 1}\left(\left\|Y_{n}\right\|-\varepsilon n^{\alpha}\right)_{+}>\varepsilon v_{1}^{1 / \beta}\right\} \\
& \leq \sum_{k=1}^{\infty} \mathbb{P}\left\{\max _{\left(2^{k-1}-1\right) v_{1}^{1 / \beta}<n^{\alpha} \leq 2^{k} v_{1}^{1 / \beta}}\left(\left\|Y_{n}\right\|-\varepsilon n^{\alpha}\right)_{+}>\varepsilon v_{1}^{1 / \beta}\right\} \\
& \leq \sum_{k=1}^{\infty} \mathbb{P}\left\{Y_{\left(2^{k} v_{1}^{1 / \beta}\right)^{1 / \alpha}}^{*}>2^{k-1} \varepsilon v_{1}^{1 / \beta}\right\} \\
& =\sum_{k=1}^{\infty} \mathbb{P}\left\{Y_{2^{k / \alpha}}^{*} v_{1}^{1 / b}>2^{k-1} \varepsilon v_{1}^{\alpha / b}\right\} \\
& \leq \sum_{k=1}^{\infty} \frac{\delta}{\phi\left(2^{k / \alpha} v_{1}^{1 / b}\right)} \text {. }
\end{aligned}
$$

Set $v=v_{1}^{1 / b}$. Since $l$ is slowly varying at $\infty$, by Potter's Theorem (cf. Theorem 1.5.6 in [39, page 25]), for $A=2$ and $\delta_{1}=b / 2>0$, there exists $v_{3}=v_{3}(b, \alpha)$ such that for all $v \geq v_{3}, l(v) / l\left(2^{k / \alpha} v\right) \leq 2\left(2^{k / \alpha}\right)^{\delta_{1}}=2 \cdot 2^{b k / 2 \alpha}$. Thus, there exists $v_{0}^{\prime}=v_{0}^{\prime}\left(v_{0}, b, \alpha\right)=\max \left\{v_{2}^{1 / b}, v_{3}\right\}>0$ such that for all $v \geq v_{0}^{\prime}$,

$$
\begin{aligned}
\phi(v) \mathbb{P}\left\{\sup _{n \geq 1}\left(Y_{n}^{*}-\varepsilon n^{\alpha}\right)_{+}>\varepsilon v^{\alpha}\right\} & \leq \delta \sum_{k=1}^{\infty} \frac{\phi(v)}{\phi\left(2^{k / \alpha} v\right)} \\
& =\delta \sum_{k=1}^{\infty} \frac{2^{-b k / \alpha} l(v)}{l\left(2^{k / \alpha} v\right)} \\
& \leq 2 \delta \sum_{k=1}^{\infty} 2^{-b k /(2 \alpha)} \\
& =\delta C(b, \alpha) .
\end{aligned}
$$

Theorem 22. Let $\alpha, b>0$ and $\phi(n)=n^{b} l(n)$. Then, the following assertions are equivalent:

$$
\phi(n) \mathbb{P}\left\{Y_{n}^{*}>\varepsilon n^{\alpha}\right\}=o(1) \quad(\text { resp., } O(1)) \text { for any } \varepsilon>0
$$

$$
\begin{gathered}
\phi(n) \mathbb{P}\left\{\sup _{j \geq n} j^{-\alpha} Y_{j}^{*}>\varepsilon\right\}=o(1) \\
(\text { resp., } O(1)) \text { for any } \varepsilon>0 ; \\
\phi(n) \mathbb{P}\left\{M(\varepsilon)>\varepsilon n^{\alpha}\right\}=o(1) \\
(\text { resp., } O(1)) \text { for any } \varepsilon>0 .
\end{gathered}
$$

Proof. We use Lemma 21. By the second inequality of (102), we see that (112) implies (111); by the first inequality of (102), we know that (111) implies (110). As (103) implies (104), we see that (110) implies (112). Thus (110), (111), and (112) are all equivalent.

Lemma 23. Let $\alpha>-1$. Then for some $n_{0}, c_{1}, c_{2}>0$ and all $N \geq n_{0}$

$$
c_{1} N^{\alpha+1} l(N) \leq \sum_{n=1}^{N} n^{\alpha} l(n) \leq c_{2} N^{\alpha+1} l(N) .
$$

Proof. Without loss of generality, we suppose that $l(s)$ has the form (101) with $c(s) \equiv 1$. Therefore, for $\delta \in(0, \alpha+1), s^{\delta} l(s)$ is increasing in $\left[n_{1}, \infty\right)$ for some $n_{1}>0$ large enough. Consequently, for some positive constants $c_{0}, c_{2}$, and $c_{3}$ (which may depend on $n_{1}$ ) and all $N \geq n_{1}$,

$$
\begin{aligned}
\sum_{n=1}^{N} n^{\alpha} l(n) & =\sum_{n=1}^{N} n^{\alpha-\delta} n^{\delta} l(n) \\
& \leq c_{0}+\sum_{n=n_{1}}^{N} n^{\alpha-\delta} n^{\delta} l(n) \\
& \leq c_{0}+N^{\alpha+1} l(N) \sum_{n=1}^{N}\left(\frac{n}{N}\right)^{\alpha-\delta} \frac{1}{N} \\
& \leq c_{0}+N^{\alpha+1} l(N) c_{3} \\
& \left(\text { as } \lim _{N \rightarrow \infty} \sum_{n=1}^{N}\left(\frac{n}{N}\right)^{\alpha-\delta} \frac{1}{N}=\int_{0}^{1} x^{\alpha-\delta} \mathrm{d} x<\infty\right) \\
& \leq c_{2} N^{\alpha+1} l(N) .
\end{aligned}
$$

Similarly, for $N \geq 2 n_{1}$,

$$
\begin{aligned}
\sum_{n=1}^{N} n^{\alpha} l(n) & \geq \sum_{N / 2 \leq n \leq N} n^{\alpha-\delta} n^{\delta} l(n) \\
& \geq\left(\frac{N}{2}\right)^{\delta} l\left(\frac{N}{2}\right) \sum_{N / 2<n \leq N} n^{\alpha-\delta} .
\end{aligned}
$$


Since $l$ is slowly varying at $\infty$, by Potter's Theorem, for $A=2$ and $\delta_{1}=1$, there exists $n_{2}$ such that for all $N \geq n_{2}, l(N / 2) \geq$ $2^{-2} l(N)$. If $\delta \leq \alpha$, then $\sum_{N / 2<n \leq N} n^{\alpha-\delta} \geq(N / 2)^{\alpha-\delta}(N-$ $[N / 2]) \geq c_{4} N^{\alpha+1-\delta}$ for some positive constant $c_{4}$; if $\delta>\alpha$, then $\sum_{N / 2<n \leq N} n^{\alpha-\delta} \geq N^{\alpha-\delta}(N-[N / 2]) \geq c_{5} N^{\alpha+1-\delta}$ for some positive constant $c_{5}$. So, for some constants $c_{1}>0$, $n_{0}=\max \left\{2 n_{1}, n_{2}\right\}$, and all $N \geq n_{0}$,

$$
\sum_{n=1}^{N} n^{\alpha} l(n) \geq c_{1} N^{\alpha+1} l(N)
$$

Theorem 24. Let $\alpha, b>0$ and $\phi(n)=n^{b-1} l(n)$. Then, the following assertions are equivalent:

$$
\begin{aligned}
& \sum_{n=1}^{\infty} \phi(n) \mathbb{P}\left\{Y_{n}^{*}>\varepsilon n^{\alpha}\right\}<\infty \quad \forall \varepsilon>0 ; \\
& \sum_{n=1}^{\infty} \phi(n) \mathbb{P}\left\{\sup _{j \geq n} j^{-\alpha} Y_{j}^{*}>\varepsilon\right\}<\infty \forall \varepsilon>0 ; \\
& \sum_{n=1}^{\infty} \phi(n) \mathbb{P}\left\{M(\varepsilon)>\varepsilon n^{\alpha}\right\}<\infty \quad \forall \varepsilon>0 ; \\
& \mathbb{E}\left(M(\varepsilon)^{b / \alpha} l\left(M(\varepsilon)^{1 / \alpha}\right)\right)<\infty \quad \forall \varepsilon>0 .
\end{aligned}
$$

Proof. We proceed as in [34, page 394] where similar results were established for $l(n)=1$ and real-valued random variables.

(a) We first prove that (117) is equivalent to

$$
\int_{1}^{\infty} \phi(u) \mathbb{P}\left\{Y_{u}^{*}>\frac{1}{2} \varepsilon u^{\alpha}\right\} \mathrm{d} u<\infty \quad \forall \varepsilon>0 .
$$

Let $n_{0}$ be large enough such that $u^{-1} l(u)$ is decreasing in $\left[n_{0}, \infty\right)$. Then, we have

$$
\begin{aligned}
& \int_{n-1}^{n} \phi(u) \mathbb{P}\left\{Y_{u}^{*}>\frac{1}{2} \varepsilon u^{\alpha}\right\} \mathrm{d} u \\
& \quad=\int_{n-1}^{n} u^{b}\left(u^{-1} l(u)\right) \mathbb{P}\left\{Y_{u}^{*}>\frac{1}{2} \varepsilon u^{\alpha}\right\} \mathrm{d} u \\
& \quad \leq n^{b}\left((n-1)^{-1} l(n-1)\right) \mathbb{P}\left\{Y_{n}^{*}>\frac{1}{2} \varepsilon(n-1)^{\alpha}\right\} \\
& \quad \leq 2 \phi(n) \mathbb{P}\left\{Y_{n}^{*}>\frac{1}{4} \varepsilon n^{\alpha}\right\}
\end{aligned}
$$

for $n \geq n_{1} \geq n_{0}+1$ large enough. Here we have used the fact that $\lim _{n \rightarrow \infty}(l(n-1) / l(n))=1$. (To see this, notice that $l(n-1) /(n-1) \geq l(n) / n$ for $n \geq n_{0}+1$, so that $l(n-1) / l(n) \geq(n-$ $1) / n$; in the same way, using the fact that $u l(u)$ is increasing in $\left[n_{0}^{\prime}, \infty\right)$ for $n_{0}^{\prime}$ large enough, we obtain $l(n-1) / l(n) \leq n /(n-1)$ for all $n \geq n_{0}^{\prime}+1$.) Similarly,

$$
\begin{aligned}
& \int_{n-1}^{n} \phi(u) \mathbb{P}\left\{Y_{u}^{*}>\frac{1}{2} \varepsilon u^{\alpha}\right\} \mathrm{d} u \\
& =\int_{n-1}^{n} u^{b}\left(u^{-1} l(u)\right) \mathbb{P}\left\{Y_{u}^{*}>\frac{1}{2} \varepsilon u^{\alpha}\right\} \mathrm{d} u \\
& \geq(n-1)^{b}\left(n^{-1} l(n)\right) \mathbb{P}\left\{Y_{n-1}^{*}>\frac{1}{2} \varepsilon n^{\alpha}\right\} \\
& \geq \frac{1}{2} \phi(n-1) \mathbb{P}\left\{Y_{n-1}^{*}>\varepsilon(n-1)^{\alpha}\right\}
\end{aligned}
$$

for $n \geq n_{2} \geq n_{0}+1$ large enough. Let $N=\max \left\{n_{1}, n_{2}\right\}$. As $\int_{N-1}^{\infty}=\sum_{n=N}^{\infty} \int_{n-1}^{n}$, the conclusion that (117) is equivalent to (121) follows from (122) and (123).

(b) We next remark that (119) is equivalent to

$$
\int_{1}^{\infty} u^{b-1} l(u) \mathbb{P}\left\{M(\varepsilon)>\varepsilon u^{\alpha}\right\} \mathrm{d} u<\infty \quad \forall \varepsilon>0 .
$$

This can be seen by the same argument as in (a).

(c) We now prove that (121) implies (124). Set $\beta=b / \alpha$. We have

$$
\begin{aligned}
& \int_{1}^{\infty} u^{b-1} l(u) \mathbb{P}\left\{M(\varepsilon)>\varepsilon u^{\alpha}\right\} \mathrm{d} u \\
& =\frac{1}{b} \int_{1}^{\infty} l\left(v^{1 /(\alpha \beta)}\right) \mathbb{P}\left\{\sup _{j \geq 1}\left(\left\|Y_{j}\right\|-\varepsilon j^{\alpha}\right)_{+}>\varepsilon v^{1 / \beta}\right\} \mathrm{d} v \\
& \leq \frac{1}{b} \int_{1}^{\infty} l\left(v^{1 /(\alpha \beta)}\right) \\
& \times \sum_{k=1}^{\infty} \mathbb{P}\left\{\max _{\left(2^{k-1}-1\right) v^{1 / \beta}<j^{\alpha} \leq 2^{k} v^{1 / \beta}}\left(\left\|Y_{j}\right\|-\varepsilon j^{\alpha}\right)_{+}>\varepsilon v^{1 / \beta}\right\} \mathrm{d} v
\end{aligned}
$$

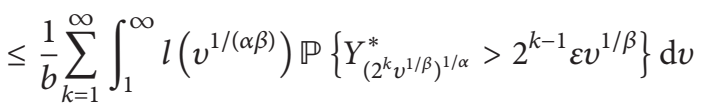

$$
\begin{aligned}
& =\sum_{k=1}^{\infty} 2^{-k \beta} \int_{1}^{\infty} u^{b-1} l\left(2^{-k / \alpha} u\right) \mathbb{P}\left\{Y_{u}^{*}>\frac{1}{2} \varepsilon u^{\alpha}\right\} \mathrm{d} u .
\end{aligned}
$$

By Potter's Theorem, for $0<\delta<b$, there exists $A=A(\delta)>1$ such that

$$
l\left(2^{-k / \alpha} u\right) \leq A 2^{k \delta / \alpha} l(u) \quad \forall k \geq 1
$$

So,

$$
\begin{aligned}
& \int_{1}^{\infty} u^{b-1} l(u) \mathbb{P}\left\{M(\varepsilon)>\varepsilon u^{\alpha}\right\} \mathrm{d} u \\
& \quad \leq A \sum_{k=1}^{\infty} 2^{-k(\beta-\delta / \alpha)} \int_{1}^{\infty} u^{b-1} l(u) \mathbb{P}\left\{Y_{u}^{*}>\frac{1}{2} \varepsilon u^{\alpha}\right\} \mathrm{d} u \\
& \quad=\frac{A}{2^{(b-\delta) / \alpha}-1} \int_{1}^{\infty} \phi(u) \mathbb{P}\left\{Y_{u}^{*}>\frac{1}{2} \varepsilon u^{\alpha}\right\} \mathrm{d} u .
\end{aligned}
$$

Thus, (121) implies (124). 
(d) We then conclude that (117), (118), and (119) are equivalent. By (a), (b), and (c), we see that (117) implies (119). By Lemma 21, we have the implications: $(119) \Rightarrow(118) \Rightarrow(117)$. have

(e) We finally prove that (119) and (120) are equivalent. We

$$
\begin{aligned}
\sum_{n=1}^{\infty} \phi(n) \mathbb{P}\left\{M(\varepsilon)>\varepsilon n^{\alpha}\right\} & =\mathbb{E}\left(\sum_{n=1}^{\infty} \phi(n) \mathbf{1}_{\left\{M(\varepsilon)>\varepsilon n^{\alpha}\right\}}\right) \\
& =\mathbb{E}\left(\sum_{1 \leq n<(M(\varepsilon) / \varepsilon)^{1 / \alpha}} l(n) n^{b-1}\right) .
\end{aligned}
$$

By Lemma 23, there exist $n_{0}, c_{1}>0$ such that

$$
\begin{aligned}
& \mathbb{E}\left(\left(\sum_{1 \leq n<(M(\varepsilon) / \varepsilon)^{1 / \alpha}} l(n) n^{b-1}\right) \mathbf{1}_{\left\{(M(\varepsilon) / \varepsilon)^{1 / \alpha} \geq n_{0}\right\}}\right) \\
& \quad \geq c_{1} \mathbb{E}\left(\left(\frac{M(\varepsilon)}{\varepsilon}\right)^{b / \alpha} l\left(\left(\frac{M(\varepsilon)}{\varepsilon}\right)^{1 / \alpha}\right) \mathbf{1}_{\left\{(M(\varepsilon) / \varepsilon)^{1 / \alpha} \geq n_{0}\right\}}\right) .
\end{aligned}
$$

By (128) and (129), we see that (119) implies

$$
\mathbb{E}\left(\left(\frac{M(\varepsilon)}{\varepsilon}\right)^{b / \alpha} l\left(\left(\frac{M(\varepsilon)}{\varepsilon}\right)^{1 / \alpha}\right)\right)<\infty \quad \forall \varepsilon>0 .
$$

By Potter's Theorem, there exists $x_{0}>0$ such that when $(M(\varepsilon) / \varepsilon)^{1 / \alpha} \geq x_{0}$ and $(M(\varepsilon))^{1 / \alpha} \geq x_{0}$,

$$
\begin{aligned}
\frac{l\left(M(\varepsilon)^{1 / \alpha}\right)}{2 \max \left\{\varepsilon^{-1 / \alpha}, \varepsilon^{1 / \alpha}\right\}} & \leq l\left(\left(\frac{M(\varepsilon)}{\varepsilon}\right)^{1 / \alpha}\right) \\
& \leq 2 \max \left\{\varepsilon^{-1 / \alpha}, \varepsilon^{1 / \alpha}\right\} l\left(M(\varepsilon)^{1 / \alpha}\right) .
\end{aligned}
$$

Therefore (130), is equivalent to (120). Hence, (119) implies (120). A similar argument (using again Lemma 23) shows that (120) implies (119). Thus, (119) and (120) are equivalent.

\section{Convergence Rates for Banach Valued Martingales}

In this section, we consider the convergence rate in the law of large numbers for a sequence of Banach valued martingales. We will obtain more equivalent conditions than in Section 4, using the results of Section 5.

Let $(\mathbb{B},\|\cdot\|)$ a separable Banach space and $\left\{\left(X_{j}, \mathscr{F}_{j}\right)\right\}_{j \geq 1}$ a sequence of $\mathbb{B}$-valued martingale differences. We denote

$$
S_{0}=0, \quad S_{n}=\sum_{j=1}^{n} X_{j}, \quad S_{n}^{*}=\max _{0 \leq j \leq n}\left\|S_{j}\right\| \quad \forall n \in \mathbb{N} .
$$

Notice that $\left\|S_{0}\right\|=0, S_{0}^{*}=0$ by our notations. Set

$$
X_{0}=0, \quad X_{n}^{*}=\max _{0 \leq j \leq n}\left\|X_{j}\right\| \quad \forall n \in \mathbb{N} .
$$

Notice that $\left\|S_{n}\right\| / n^{\alpha} \rightarrow 0$ a.s. if and only if $\mathbb{P}\left(\sup _{j \geq n} j^{-\alpha}\left\|S_{j}\right\|>\right.$ $\varepsilon) \rightarrow 0$ for any $\varepsilon>0$. So, the following theorems describe the a.s. convergence of $\left\|S_{n}\right\| / n^{\alpha}$.

Theorem 25. Let $\alpha, b>0, l(\cdot)>0$ be a function slowly varying at $\infty$ and $\phi(n)=n^{b} l(n)$. Suppose that for some $\gamma \in(1,2], q \in$ $[1, \infty]$ and $\lambda \in(0, q)$,

$$
\phi(n)\left\|m_{n}(\gamma)\right\|_{\mathbb{L}_{\boxplus}^{q}}^{\lambda}=O(1)
$$

where $m_{n}(\gamma)=n^{-\gamma \alpha} \sum_{j=1}^{n} \mathbb{E}\left[\left\|X_{j}\right\|^{\gamma} \mid \mathscr{F}_{j-1}\right]$. If $\mathbb{B}$ is $\gamma$-smooth, then one has the following implications $(135) \Leftrightarrow(136) \Leftrightarrow(137) \Leftrightarrow$ $(140) \Rightarrow(139) \Rightarrow(138)$ :

$$
\begin{gathered}
\phi(n) \sum_{j=1}^{n} \mathbb{P}\left\{\left\|X_{j}\right\|>\varepsilon n^{\alpha}\right\}=o(1) \\
(\text { resp., } O(1)) \text { for any } \varepsilon>0 ; \\
\phi(n) P\left\{X_{n}^{*}>\varepsilon n^{\alpha}\right\}=o(1) \quad(\text { resp., } O(1)) \text { for any } \varepsilon>0 ;
\end{gathered}
$$

$$
\phi(n) P\left\{S_{n}^{*}>\varepsilon n^{\alpha}\right\}=o(1) \quad(\text { resp., } O(1)) \text { for any } \varepsilon>0
$$

$\phi(n) P\left\{\left\|S_{n}\right\|>\varepsilon n^{\alpha}\right\}=o(1)$

(resp., $O(1))$ for any $\varepsilon>0$;

$$
\begin{array}{r}
\phi(n) P\left\{\sup _{j \geq n} j^{-\alpha}\left\|S_{j}\right\|>\varepsilon\right\}=o(1) \\
(\text { resp., } O(1)) \text { for any } \varepsilon>0 ; \\
\phi(n) P\left\{\sup _{j \geq n} j^{-\alpha} S_{j}^{*}>\varepsilon\right\}=o(1)
\end{array}
$$

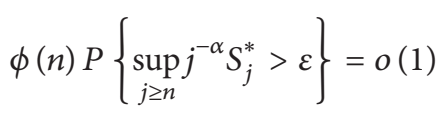

(resp., $O(1))$ for any $\varepsilon>0$. 
Corollary 27. Let $1 / 2<\alpha \leq 1$ and $b>0$. Let $l(\cdot)>0$ be a function slowly varying at $\infty$ and $\phi(n)=n^{b} l(n)$. Suppose that for some $\gamma \in(1 / \alpha, 2], q \in[1, \infty]$ with $q>b /(\gamma \alpha-1)$,

$$
\begin{aligned}
& \sup _{n \geq 1}\left\|\underline{m}_{n}(\gamma)\right\|_{\underline{L}_{\mathbb{B}}^{q}}<\infty, \\
& \text { where } \underline{m}_{n}(\gamma)=\frac{1}{n} \sum_{j=1}^{n} \mathbb{E}\left[\left\|X_{j}\right\|^{\gamma} \mid \mathscr{F}_{j-1}\right] .
\end{aligned}
$$

If $\mathbb{B}$ is $\gamma$-smooth, then one has the implications $(135) \Leftrightarrow(136) \Leftrightarrow$ $(137) \Leftrightarrow(140) \Rightarrow(139) \Rightarrow(138)$.

Proof of Theorem 25. Applying Theorem 14 to $X_{n j}=X_{j}$, $\mathscr{F}_{n j}=\mathscr{F}_{j}(1 \leq j \leq n)$, and $\phi(n)=n^{b} l(n)$, we get the implications $(135) \Leftrightarrow(136) \Leftrightarrow(137) \Rightarrow(138)$. Applying Theorem 22 to $Y_{n}=\left\|S_{n}\right\|$, we know that (137) and (140) are equivalent. Obviously, we have the implications $(140) \Rightarrow(139) \Rightarrow(138)$. Therefore, we have proved the implications $(135) \Leftrightarrow(136) \Leftrightarrow(137) \Leftrightarrow(140) \Rightarrow(139) \Rightarrow(138)$.

Proof of Corollary 27. Since $\gamma>1 / \alpha$, we have

$$
\left\|m_{n}(\gamma)\right\|_{\mathbb{L}_{B}^{q}}=n^{1-\gamma \alpha}\left\|\underline{m}_{n}(\gamma)\right\|_{\mathbb{L}_{B}^{q}} .
$$

As $q>b /(\gamma \alpha-1)$, we can choose $\lambda \in(b /(\gamma \alpha-1), q)$. For this $\lambda$,

$$
\begin{aligned}
\phi(n)\left\|m_{n}(\gamma)\right\|_{\mathbb{L}_{\mathbb{B}}^{q}}^{\lambda} & =n^{b} l(n)\left(n^{1-\gamma \alpha}\left\|\underline{m}_{n}(\gamma)\right\|_{\mathbb{L}_{\mathbb{B}}^{q}}\right)^{\lambda} \\
& =n^{b-\lambda(\gamma \alpha-1)} l(n)\left\|\underline{m}_{n}(\gamma)\right\|_{\mathbb{L}_{\mathbb{B}}^{q}}^{\lambda}=o(1) .
\end{aligned}
$$

Thus, the condition (134) holds, so that the conclusion follows from Theorem 25.

Theorem 28. Let $\alpha, b>0$. Let $l(\cdot)>0$ be a function slowly varying at $\infty$ and $\phi(n)=n^{b-1} l(n)$. For any $\varepsilon>0$, set

$$
\begin{aligned}
M(S, \varepsilon) & =M(S, \varepsilon, \alpha)=\sup _{n \geq 0}\left(\left\|S_{n}\right\|-\varepsilon n^{\alpha}\right) \\
& =\sup _{n \geq 0}\left(S_{n}^{*}-\varepsilon n^{\alpha}\right), \\
M(X, \varepsilon) & =M(X, \varepsilon, \alpha)=\sup _{n \geq 0}\left(\left\|X_{n}\right\|-\varepsilon n^{\alpha}\right) \\
& =\sup _{n \geq 0}\left(X_{n}^{*}-\varepsilon n^{\alpha}\right) .
\end{aligned}
$$

Suppose that for some $\gamma \in(1,2], q \in[1, \infty]$ and $\lambda \in(0, q)$,

$$
\left\|m_{n}(\gamma)\right\|_{\mathbb{L}_{\mathbb{B}}^{q}} \longrightarrow 0, \quad \sum_{n=1}^{\infty} \phi(n)\left\|m_{n}(\gamma)\right\|_{\mathbb{L}_{\mathbb{B}}^{q}}^{\lambda}<\infty,
$$

where $m_{n}(\gamma)=n^{-\gamma \alpha} \sum_{j=1}^{n} \mathbb{E}\left[\left\|X_{j}\right\|^{\gamma} \mid \mathscr{F}_{j-1}\right]$. If $\mathbb{B}$ is $\gamma$-smooth, then one has the following implications $(153) \Leftrightarrow(154) \Leftrightarrow(155) \Leftrightarrow$ $(156) \Leftrightarrow(148) \Leftrightarrow(149) \Leftrightarrow(150) \Rightarrow(151) \Rightarrow(152)$ :
(A)

$$
\begin{gathered}
\sum_{n=1}^{\infty} \phi(n) \mathbb{P}\left\{S_{n}^{*}>\varepsilon n^{\alpha}\right\}<\infty \quad \forall \varepsilon>0 ; \\
\sum_{n=1}^{\infty} \phi(n) \mathbb{P}\left\{\sup _{j \geq n} j^{-\alpha} S_{j}^{*}>\varepsilon\right\}<\infty \quad \forall \varepsilon>0 ; \\
\mathbb{E}\left(M(S, \varepsilon)^{b / \alpha} l\left(M(S, \varepsilon)^{1 / \alpha}\right)\right)<\infty \quad \forall \varepsilon>0 ; \\
\sum_{n=1}^{\infty} \phi(n) \mathbb{P}\left\{\sup _{j \geq n}^{-\alpha}\left\|S_{j}\right\|>\varepsilon\right\}<\infty \quad \forall \varepsilon>0 ; \\
\sum_{n=1}^{\infty} \phi(n) \mathbb{P}\left\{\left\|S_{n}\right\|>\varepsilon n^{\alpha}\right\}<\infty \quad \forall \varepsilon>0 ;
\end{gathered}
$$

(B)

$$
\begin{gathered}
\sum_{n=1}^{\infty} \phi(n) \mathbb{P}\left\{X_{n}^{*}>\varepsilon n^{\alpha}\right\}<\infty \quad \forall \varepsilon>0 ; \\
\sum_{n=1}^{\infty} \phi(n) \mathbb{P}\left\{\sup _{j \geq n} j^{-\alpha} X_{j}^{*}>\varepsilon\right\}<\infty \quad \forall \varepsilon>0 ; \\
\mathbb{E}\left(M(X, \varepsilon)^{b / \alpha} l\left(M(X, \varepsilon)^{1 / \alpha}\right)\right)<\infty \quad \forall \varepsilon>0 ; \\
\sum_{n=1}^{\infty} \phi(n) \sum_{j=1}^{n} \mathbb{P}\left\{\left\|X_{j}\right\|>\varepsilon n^{\alpha}\right\}<\infty \quad \forall \varepsilon>0 .
\end{gathered}
$$

Compared with Theorem 17, in Theorem 28 we have the additional conditions (149), (150), (151), (154), and (155).

Remark 29. As in Theorem 25, the conclusions of Theorem 28 remain valid if $m_{n}(\gamma)$ is replaced by

$$
m_{n}(\gamma, M)=n^{-\gamma \alpha} \sum_{j=1}^{n} \mathbb{E}\left[\left\|X_{j}\right\|^{\gamma} \mid \mathscr{F}_{j-M}\right]
$$

for some $M \in \mathbb{N}^{*}$.

Corollary 30. Let $\alpha>1 / 2, b>0$. Let $l(\cdot)>0$ be a function slowly varying at $\infty$ and $\phi(n)=n^{b-1} l(n)$. Suppose that for some $\gamma \in(\max \{1,1 / \alpha\}, 2], q \in[1, \infty]$ with $q>b /(\gamma \alpha-1)$, (142) holds. If $\mathbb{B}$ is $\gamma$-smooth, then one has the implications $(153) \Leftrightarrow(154) \Leftrightarrow(155) \Leftrightarrow(156) \Leftrightarrow(148) \Leftrightarrow(149) \Leftrightarrow(150) \Rightarrow(151) \Rightarrow$ (152).

If $X_{j}$ 's are identically distributed real-valued random variables, then (156) is equivalent to the moment condition $\mathbb{E}\left|X_{1}\right|^{(b+1) / \alpha} l\left(\left|X_{1}\right|^{1 / \alpha}\right)<\infty$. So, Corollary 30 contains Theorems 1, 2, and 3 of Baum and Katz [6] when $\phi(n)=n^{b-1}$ and for i.i.d. real-valued random variables. When $\phi(n)=n^{b-1}, \alpha \leq$ 1 and for real-valued martingale differences, Corollary 30 was proved by Alsmeyer [18, Theorems 1 and 2].

To see that Theorem 28 implies Corollary 30 , it suffices to notice that (142) (with $q>b /(\gamma \alpha-1)$ ) implies (147). In fact, when (142) holds for some $q \in[1, \infty]$ with $q>b /(\gamma \alpha-1)$, then

$$
\left\|m_{n}(\gamma)\right\|_{\mathbb{L}_{\mathbb{B}}^{q}}=n^{1-\gamma \alpha}\left\|\underline{m}_{n}(\gamma)\right\|_{\mathbb{L}_{\mathbb{B}}^{q}} \longrightarrow 0,
$$


and, for $\lambda \in(b /(\gamma \alpha-1), q)$,

$$
\begin{aligned}
& \sum_{n=1}^{\infty} \phi(n)\left\|m_{n}(\gamma)\right\|_{\mathbb{B}_{\mathbb{B}}^{q}}^{\lambda} \\
& \quad=\sum_{n=1}^{\infty} n^{b-1-\lambda(\gamma \alpha-1)} l(n)\left\|\underline{m}_{n}(\gamma)\right\|_{\mathbb{L}_{\mathbb{B}}^{q}}^{\lambda}<\infty .
\end{aligned}
$$

Therefore, (147) holds for some $q \in[1, \infty]$ and $\lambda \in(0, q)$.

Proof of Theorem 28. Applying Theorem 10 to

$$
\begin{aligned}
& X_{n j}= \begin{cases}n^{-\alpha} X_{j} & \text { if } 1 \leq j \leq n, \\
0 & \text { if } j>n,\end{cases} \\
& \mathscr{F}_{n j}= \begin{cases}\mathscr{F}_{j} & \text { if } 1 \leq j \leq n, \\
\mathscr{F}_{n} & \text { if } j>n,\end{cases}
\end{aligned}
$$

we obtain the implications $(153) \Leftrightarrow(156) \Leftrightarrow(148) \Rightarrow(152)$. By Theorem 24 applied to $Y_{n}=\left\|S_{n}\right\|$, we see that (148), (149), and (150) are equivalent. Since $n^{-\alpha}\left\|S_{n}\right\| \leq$ $\sup _{j \geq n} j^{-\alpha}\left\|S_{j}\right\| \leq \sup _{j \geq n} j^{-\alpha} S_{j}^{*}$, (149) implies (151) and (151) implies (152). Thus, we have the implications $(148) \Leftrightarrow$ $(149) \Leftrightarrow(150) \Rightarrow(151) \Rightarrow(152)$. Again by Theorem 24 applied to $Y_{n}=\left\|X_{n}\right\|$, we know that (153), (154), and (155) are equivalent. Therefore, we have the implications $(153) \Leftrightarrow(154) \Leftrightarrow(155) \Leftrightarrow$ $(156) \Leftrightarrow(148) \Leftrightarrow(149) \Leftrightarrow(150) \Rightarrow(151) \Rightarrow(152)$.

\section{Convergence Rates for Weighted Sums of Banach Valued Martingale Differences of the Form $\sum_{j=1}^{n} j^{a-1} X_{j}$}

Let $(\mathbb{B},\|\cdot\|)$ be a separable Banach space. In this section, we give a Marcinkiewicz-Zygmund type strong law of large numbers for weighted sums

$$
T_{n}=\sum_{j=1}^{n} j^{a-1} X_{j} \quad(a \in \mathbb{R})
$$

of $\mathbb{B}$-valued martingale differences $\left\{\left(X_{j}, \mathscr{F}_{j}\right)\right\}_{j \geq 1}$, and we obtain a Baum-Katz type theorem for weighted sums of identically distributed $\mathbb{B}$-valued martingale differences which extends Theorems 2 and 3 of Lanzinger and Stadtmüller [21]. Our results will be obtained by means of our main Theorems 2 and 10.

We will need the following elementary result.

Lemma 31. Let $\left(Y_{j}\right)_{j \geq 1}$ be a sequence of any $\mathbb{B}$-valued random variables. If there exist $n_{0}, K>0$, such that for some $q \in[1, \infty]$ and all $n \geq n_{0}$,

$$
\left\|\frac{1}{n} \sum_{j=1}^{n} Y_{j}\right\|_{\mathbb{L}_{\mathbb{B}}^{q}} \leq K
$$

then

$$
\left\|\sum_{j=1}^{n} j^{p} Y_{j}\right\|_{\mathbb{L}_{\mathbb{B}}^{q}}=O\left(n^{p+1}\right) \quad \text { for any } p>-1 .
$$

Proof. Let $S_{0}=0, S_{n}=\sum_{j=1}^{n} Y_{j}, n \geq 1$. Then,

$$
\begin{aligned}
\sum_{j=1}^{n} j^{p} Y_{j} & =\sum_{j=1}^{n} j^{p}\left(S_{j}-S_{j-1}\right) \\
& =\sum_{j=1}^{n-1}\left[j^{p}-(j+1)^{p}\right] S_{j}+n^{p} S_{n} \\
& =\sum_{j=1}^{n-1} j^{p}\left[1-\left(1+\frac{1}{j}\right)^{p}\right] S_{j}+n^{p} S_{n} .
\end{aligned}
$$

As $1-(1+1 / j)^{p} \sim-p / j \quad(j \rightarrow \infty)$, it follows that

$$
\begin{aligned}
& \left\|\sum_{j=1}^{n} j^{p} Y_{j}\right\|_{\mathbb{L}_{\mathbb{B}}^{q}} \\
& \quad \leq \sum_{j=1}^{n-1} j^{p}\left|1-\left(1+\frac{1}{j}\right)^{p}\right|\left\|S_{j}\right\|_{\mathbb{L}_{\mathbb{B}}^{q}}+n^{p}\left\|S_{n}\right\|_{\mathbb{L}_{\mathbb{B}}^{q}} \\
& \quad=O\left(n^{p+1}\right) .
\end{aligned}
$$

The following theorem is a Marcinkiewicz-Zygmund type strong law of large numbers for the weighted sums (161).

Theorem 32. Let $\left\{\left(X_{j}, \mathscr{F}_{j}\right)\right\}_{j \geq 1}$ be $\mathbb{B}$-valued martingale differences. Suppose that for some $\gamma \in(1,2]$, there exist $n_{0}, K>0$, such that for all $n \geq n_{0}$,

$$
\frac{1}{n} \sum_{j=1}^{n} \mathbb{E}\left\|X_{j}\right\|^{\gamma} \leq K
$$

If $\mathbb{B}$ is $\gamma$-smooth, then for $\alpha>-(1-1 / \gamma)$ and $a>1-1 / \gamma$,

$$
\begin{gathered}
\frac{T_{n}}{n^{a+\alpha}} \longrightarrow 0, \text { a.s., } \\
\mathbb{P}\left\{\sup _{m \geq n} \frac{\left\|T_{m}\right\|}{m^{a+\alpha}}>\varepsilon\right\}=O\left(n^{-(\gamma-1+\gamma \alpha)}\right) \quad \text { for any } \varepsilon>0 .
\end{gathered}
$$

Notice that when $a=1$ and for real-valued martingale differences, the result (167) is implied by the classical Marcinkiewicz-Zygmund strong laws of large numbers. Also, it is evident that (167) holds if and only if $\mathbb{P}\left\{\sup _{m \geq n}\left(\left\|T_{m}\right\| / m^{a+\alpha}\right)>\varepsilon\right\} \rightarrow 0$ for any $\varepsilon>0$. So, (168) describes the convergence rates in the MarcinkiewiczZygmund strong laws of large numbers (167).

Proof of Theorem 32. Clearly,

$$
\mathbb{P}\left\{\sup _{m \geq n} \frac{\left\|T_{m}\right\|}{m^{a+\alpha}}>\varepsilon\right\} \leq \mathbb{P}\left\{\sup _{m \geq n}\left(m^{-(a+\alpha)} \max _{1 \leq j \leq m}\left\|T_{j}\right\|\right)>\varepsilon\right\} .
$$

By Theorem 22, we see that

$$
\mathbb{P}\left\{\sup _{m \geq n}\left(m^{-(a+\alpha)} \max _{1 \leq j \leq m}\left\|T_{j}\right\|\right)>\varepsilon\right\}=O\left(n^{-(\gamma-1+\gamma \alpha)}\right) \quad \forall \varepsilon>0
$$


if and only if

$$
\mathbb{P}\left\{\max _{1 \leq j \leq n}\left\|T_{j}\right\|>\varepsilon n^{a+\alpha}\right\}=O\left(n^{-(\gamma-1+\gamma \alpha)}\right) \quad \forall \varepsilon>0 .
$$

Write $m(\gamma, n)=n^{-\gamma(a+\alpha)} \sum_{j=1}^{n} \mathbb{E}\left[\left\|j^{a-1} X_{j}\right\|^{\gamma} \mid \mathscr{F}_{j-1}\right]$. By the proof of Lemma 31, we have

$$
\begin{aligned}
\mathbb{E} m(\gamma, n) & =n^{-\gamma(a+\alpha)} \sum_{j=1}^{n} \mathbb{E}\left\|j^{a-1} X_{j}\right\|^{\gamma} \\
& =n^{-\gamma(a+\alpha)} \sum_{j=1}^{n} j^{\gamma(a-1)} \mathbb{E}\left\|X_{j}\right\|^{\gamma} \\
& =O\left(n^{-(\gamma-1+\gamma \alpha)}\right) .
\end{aligned}
$$

And by Theorem 2 with $q=1$ and $L=0$, we know that

$$
\begin{aligned}
& \mathbb{P}\left\{n^{-(a+\alpha)} \max _{1 \leq j \leq n}\left\|T_{j}\right\|>\varepsilon\right\} \\
& \leq \mathbb{P}\left\{n^{-(a+\alpha)} \max _{1 \leq j \leq n}\left\|j^{a-1} X_{j}\right\|>\frac{1}{4} \varepsilon\right\}+\varepsilon^{-\gamma} C_{2}(\gamma) \mathbb{E} m(\gamma, n) \\
& \quad=\mathbb{P}\left\{\max _{1 \leq j \leq n}\left\|j^{a-1} X_{j}\right\|>\frac{1}{4} \varepsilon n^{a+\alpha}\right\}+O\left(n^{-(\gamma-1+\gamma \alpha)}\right) .
\end{aligned}
$$

By Theorem 1, we see that

$$
\begin{aligned}
& \mathbb{P}\left\{n^{-(a+\alpha)} \max _{1 \leq j \leq n}\left\|j^{a-1} X_{j}\right\|>\frac{1}{4} \varepsilon\right\} \\
& \leq \sum_{j=1}^{n} \mathbb{P}\left\{n^{-(a+\alpha)}\left\|j^{a-1} X_{j}\right\|>\frac{1}{4} \varepsilon\right\} .
\end{aligned}
$$

By (166), we have

$$
\begin{aligned}
\sum_{j=1}^{n} \mathbb{P} & \left\{\left\|j^{a-1} X_{j}\right\|>\frac{1}{4} \varepsilon n^{a+\alpha}\right\} \\
& \leq 4^{\gamma} \varepsilon^{-\gamma} n^{-\gamma(a+\alpha)} \sum_{j=1}^{n} j^{\gamma(a-1)} \mathbb{E}\left\|X_{j}\right\|^{\gamma} \\
& =O\left(n^{-(\gamma-1+\gamma \alpha)}\right) .
\end{aligned}
$$

From (173)-(175), we see that (171) holds. Thus, (170) holds, so that (168) holds.

To establish a general Baum-Katz type theorem for the weighted sums (161), we first introduce a definition and a technical lemma.

Definition 33. For a function $R_{\rho}$ regularly varying at $\infty$ of index $\rho \neq 0$, one define, $R_{\rho}^{\leftarrow}$ as its inverse function.

Notice that when $\rho>0, R_{\rho}(x)$ is strictly increasing for $x$ large enough with $\lim _{x \rightarrow \infty} R_{\rho}(x)=+\infty$, so that $R_{\rho}^{\leftarrow}$ is well defined on $\left[u_{0}, \infty\right)$ for $u_{0}>0$ large enough. For simplicity, we always make the convention that $R_{\rho}^{\leftarrow}(u)=R_{\rho}^{\leftarrow}\left(u_{0}\right)$ if $u \in\left[0, u_{0}\right)$, so that $R_{\rho}^{\leftarrow}$ is well defined on $[0, \infty)$. We make a similar convention in the case where $\rho<0$.

The following lemma shows that the inverse function of a regularly varying function of index $\rho \neq 0$ remains regularly varying.

Lemma 34. If $R_{\rho}(x)=x^{\rho} l(x)$ is regularly varying at $\infty$ of index $\rho \neq 0$, where $l(x)$ is of the canonical form $l(x)=$ $\exp \left\{\int_{x_{0}}^{x}((\varepsilon(u)) / u) d u\right\}\left(x \geq x_{0}\right)$ for some $x_{0}>0$, then its inverse function $R_{\rho}^{\leftarrow}(x)=x^{1 / \rho} l^{*}(x)$ is regularly varying at $\infty$ of index $1 / \rho$, where $l^{*}(x)=\left(l\left(R_{\rho}^{\leftarrow}(x)\right)\right)^{-1 / \rho}$ is slowly varying at $\infty$.

Proof. Let $y=R_{\rho}(x)$. Define $l_{2}(y):=l\left(R_{\rho}^{\leftarrow}(y)\right)$. We have

$$
R_{\rho}^{\leftarrow}(y)=y^{1 / \rho}\left(l_{2}(y)\right)^{-1 / \rho}=y^{1 / \rho} l^{*}(y) .
$$

We will prove that $l_{2}(y)$ is slowly varying at $\infty$. We see that

$$
l_{2}(y)=\exp \left\{\int_{x_{0}}^{R_{\rho}^{\leftarrow}(y)} \frac{\varepsilon(u)}{u} \mathrm{~d} u\right\} .
$$

After changing variable, we have

$$
\begin{aligned}
\int_{x_{0}}^{R_{\rho}^{\leftarrow}(y)} \frac{\varepsilon(u)}{u} \mathrm{~d} u & =\int_{l(1)}^{y} \frac{\varepsilon\left(R_{\rho}^{\leftarrow}(v)\right)}{R_{\rho}^{\leftarrow}(v)}\left(R_{\rho}^{\leftarrow}(v)\right)^{\prime} \mathrm{d} v \\
& =\int_{l(1)}^{y} \frac{\varepsilon\left(R_{\rho}^{\leftarrow}(v)\right)}{R_{\rho}^{\leftarrow}(v)} \frac{R_{\rho}^{\leftarrow}(v)}{v\left(\rho+\varepsilon\left(R_{\rho}^{\leftarrow}(v)\right)\right)} \mathrm{d} v \\
& =\int_{l(1)}^{y} \frac{\varepsilon\left(R_{\rho}^{\leftarrow}(v)\right)}{v\left(\rho+\varepsilon\left(R_{\rho}^{\leftarrow}(v)\right)\right)} \mathrm{d} v \\
& =\int_{l(1)}^{y} \frac{\varepsilon_{1}(v)}{v} \mathrm{~d} v
\end{aligned}
$$

where $\varepsilon_{1}(v):=\varepsilon\left(R_{\rho}^{\leftarrow}(v)\right) /\left(\rho+\varepsilon\left(R_{\rho}^{\leftarrow}(v)\right)\right) \rightarrow 0$. Thus, $l_{2}(y)=$ $\exp \left\{\int_{l(1)}^{y}\left(\varepsilon_{1}(v) / v\right) \mathrm{d} v\right\}$ is slowly varying at $\infty$ and so is $l^{*}(y)$, which proves the desired result.

In the following Baum-Katz type theorem, $l(\cdot)$ and $l_{1}(\cdot)$ are functions slowly varying at $\infty$. Without loss of generality, we suppose that $l$ and $l_{1}$ have the form (101) with $c(x) \equiv 1$. For $\alpha>-1$ and $a>-\alpha$, define

$$
R_{1+\alpha}(x)=x^{1+\alpha} l_{1}(x), \quad R_{a+\alpha}(x)=x^{a+\alpha} l_{1}(x),
$$

and let $R_{1+\alpha}^{\leftarrow}$ and $R_{a+\alpha}^{\leftarrow}$ be, respectively, the inverse functions of $R_{1+\alpha}$ and $R_{a+\alpha}$ (cf. Definition 33), which are also regularly varying by Lemma 34 . For $a \neq 1$, choose $U$ so large that

$$
l_{0}(x):=l(x)\left(l_{1}(x)\right)^{1 /(a-1)}
$$


is locally bounded in $[U, \infty)$, and set

$$
\begin{gathered}
\tilde{l}(u)=0 \quad \text { if } u \in[0, U) \\
\tilde{l}(u)=\int_{U}^{u} x^{-1} l_{0}(x) \mathrm{d} x \quad \text { if } u \in[U, \infty] .
\end{gathered}
$$

Notice that $\widetilde{l}(\infty)$ may be finite or infinite.

Theorem 35. Let $\left\{\left(X_{j}, \mathscr{F}_{j}\right)\right\}_{j \geq 1}$ be identically distributed $\mathbb{B}$ valued martingale differences and $b \geq 0$. Suppose that for some $\gamma \in(1,2]$ and $q \in[1, \infty]$ with $q>b /(\gamma(\alpha+1)-1), \gamma(\alpha+1)-1>$ 0 and $\gamma(a-1)+1>0$,

$$
\begin{array}{r}
\sup _{n \geq 1}\left\|\underline{m}_{n}(\gamma)\right\|_{\mathbb{L}_{\mathbb{B}}^{q}}<\infty, \\
\text { where } \underline{m}_{n}(\gamma)=\frac{1}{n} \sum_{j=1}^{n} \mathbb{E}\left[\left\|X_{j}\right\|^{\gamma} \mid \mathscr{F}_{j-1}\right] .
\end{array}
$$

If $\mathbb{B}$ is $\gamma$-smooth, then the following assertions hold.

(a) When $a \neq(b-\alpha) /(b+1)$,

$$
\begin{aligned}
& \sum_{n=1}^{\infty} n^{b-1} l(n) \mathbb{P}\left\{\max _{1 \leq j \leq n}\left\|T_{j}\right\|>\varepsilon n^{a+\alpha} l_{1}(n)\right\}<\infty \\
& \forall \varepsilon>0
\end{aligned}
$$

if and only if

$$
\begin{gathered}
\mathbb{E}\left(R_{1+\alpha}^{\leftarrow}\left(\left\|X_{1}\right\|\right)\right)^{b+1} l\left(R_{1+\alpha}^{\leftarrow}\left(\left\|X_{1}\right\|\right)\right)<\infty \quad \text { if } a>\frac{b-\alpha}{b+1}, \\
\mathbb{E}\left(R_{a+\alpha}^{\leftarrow}\left(\left\|X_{1}\right\|\right)\right)^{b} l\left(R_{a+\alpha}^{\leftarrow}\left(\left\|X_{1}\right\|\right)\right)<\infty \quad \text { if } a<\frac{b-\alpha}{b+1} .
\end{gathered}
$$

(b) When $a=(b-\alpha) /(b+1)$, (183) is implied by

$$
\begin{aligned}
& \mathbb{E}\left(R_{a+\alpha}^{\leftarrow}\left(\left\|X_{1}\right\|\right)\right)^{b}\left(l_{1}\left(R_{a+\alpha}^{\leftarrow}\left(\left\|X_{1}\right\|\right)\right)\right)^{1 /(1-a)} \widetilde{l}\left(R_{a+\alpha}^{\leftarrow}\left(\left\|X_{1}\right\|\right)\right) \\
& \quad<\infty,
\end{aligned}
$$

where $\widetilde{l}$ is defined in (181); conversely, if $\widetilde{l}(\infty)=\infty$ and the function $l_{0}$ defined by (180) satisfies $\lim _{u \rightarrow \infty}\left(l_{0}\left(R_{1+\alpha}^{\leftarrow}(u)\right) /\right.$ $\left.l_{0}\left(R_{a+\alpha}^{\leftarrow}(u)\right)\right)<(1+\alpha) /(a+\alpha)$, then (183) implies (185).

Remark 36. Theorem 35 also holds if (182) is replaced by

$$
\left\|m_{n}(\gamma)\right\|_{\mathbb{L}_{\mathbb{B}}^{q}} \longrightarrow 0, \quad \sum_{n=1}^{\infty} n^{b-1} l(n)\left\|m_{n}(\gamma)\right\|_{\mathbb{L}_{\mathbb{B}}^{q}}^{\lambda}<\infty
$$

for some $\gamma \in(1,2], q \in[1, \infty]$ and $\lambda \in(0, q)$, where

$$
m_{n}(\gamma)=n^{-\gamma(a+\alpha)}\left(l_{1}(n)\right)^{-\gamma} \sum_{j=1}^{n} j^{-\gamma(1-a)} \mathbb{E}\left[\left\|X_{j}\right\|^{\gamma} \mid \mathscr{F}_{j-1}\right] .
$$

Of particular interest are the cases where the slowly varying functions $l$ and $l_{1}$ are constants or powers of the logarithmic function, which will be studied in the following corollaries. We first consider the case where $l$ and $l_{1}$ are constants.

Corollary 37. Let $\left\{\left(X_{j}, \mathscr{F}_{j}\right)\right\}_{j \geq 1}$ be identically distributed $\mathbb{B}$ valued martingale differences and $b \geq 0$. Suppose that (182) holds for some $\gamma \in(1,2]$ and $q \in[1, \infty]$ with $q>b /(\gamma(\alpha+$ $1)-1), \gamma(\alpha+1)-1>0$, and $\gamma(a-1)+1>0$. If $\mathbb{B}$ is $\gamma$-smooth, then

$$
\sum_{n=1}^{\infty} n^{b-1} \mathbb{P}\left\{\max _{1 \leq j \leq n}\left\|T_{j}\right\|>\varepsilon n^{a+\alpha}\right\}<\infty \quad \forall \varepsilon>0
$$

if and only if

$$
\begin{gathered}
\mathbb{E}\left\|X_{1}\right\|^{(b+1) /(1+\alpha)}<\infty \quad \text { if } a>\frac{b-\alpha}{b+1}, \\
\mathbb{E}\left\|X_{1}\right\|^{1 /(1-a)} \log _{+}\left\|X_{1}\right\|<\infty \quad \text { if } a=\frac{b-\alpha}{b+1}, \\
\mathbb{E}\left\|X_{1}\right\|^{b /(a+\alpha)}<\infty \quad \text { if } a<\frac{b-\alpha}{b+1} .
\end{gathered}
$$

Notice that the condition on $\underline{m}_{n}(\gamma)$ implies in particular $\mathbb{E} \underline{m}_{n}(\gamma)<\infty$, giving $\mathbb{E}\left\|X_{1}\right\|^{\gamma}<\infty$. Therefore, the conclusion of the corollary is interesting only when the exponents in (189) are greater than $\gamma$.

When $\left(X_{j}\right)_{j \geq 1}$ are i.i.d. real-valued random variables and $a \in(0,1)$, we get the sufficiencies of Theorems 2 and 3(a)(i) and (iii) of Lanzinger and Stadtmüller [21] by Corollary 37.

We then consider the case where $l(x)=\left(\log _{+} x\right)^{p} \quad(p \in$ $\mathbb{R})$ and $l_{1}(x)=\left(\log _{+} x\right)^{\beta} \quad(\beta \in \mathbb{R})$.

Corollary 38. Let $\left\{\left(X_{j}, \mathscr{F}_{j}\right)\right\}_{j \geq 1}$ be identically distributed $\mathbb{B}$ valued martingale differences and $b \geq 0$. Suppose that (182) holds for some $\gamma \in(1,2]$ and $q \in[1, \infty]$ with $q>b /(\gamma(\alpha+$ $1)-1), \gamma(\alpha+1)-1>0$, and $\gamma(a-1)+1>0$. If $\mathbb{B}$ is $\gamma$-smooth, then for $\beta, p \in \mathbb{R}$,

$$
\sum_{n=2}^{\infty} n^{b-1}(\log n)^{p} \mathbb{P}\left\{\max _{1 \leq j \leq n}\left\|T_{j}\right\|>\varepsilon n^{a+\alpha}(\log n)^{\beta}\right\}<\infty
$$

$\forall \varepsilon>0$

if and only if

$$
\begin{array}{r}
\mathbb{E}\left\|X_{1}\right\|^{(b+1) /(1+\alpha)}\left(\log _{+}\left\|X_{1}\right\|\right)^{-\beta(b+1) /(1+\alpha)+p}<\infty \\
\text { if } a>\frac{b-\alpha}{b+1}, \\
\mathbb{E}\left\|X_{1}\right\|^{1 /(1-a)}\left(\log _{+}\left\|X_{1}\right\|\right)^{-\beta /(1-a)+p+1}<\infty \\
\text { if } a=\frac{b-\alpha}{b+1}, \\
\mathbb{E}\left\|X_{1}\right\|^{b /(a+\alpha)}\left(\log _{+}\left\|X_{1}\right\|\right)^{-\beta b /(a+\alpha)+p}<\infty \\
\text { if } a<\frac{b-\alpha}{b+1} .
\end{array}
$$


In the case where $\left(X_{j}\right)_{j \geq 1}$ are i.i.d. real-valued random variables and the maximum $\max _{1 \leq j \leq n}\left\|T_{j}\right\|$ is replaced by the $\left\|T_{n}\right\|$, by Corollary 38, if $a \in(0,1), \beta=0$, and $p=-1$, we get the sufficiency of Theorem 3(a)(ii) of Lanzinger and Stadtmüller [21]; if $a=0, \beta=1$, and $p<0, p \neq-1$, we get the sufficiencies of Theorem 3(b) of Lanzinger and Stadtmüller in [21].

Proof of Theorem 35 . Notice that $\widetilde{l}(u)$ is slowly varying at $\infty$ by Proposition 1.5.9a in [39, page 26]. Set

$$
\begin{gathered}
X_{n j}= \begin{cases}n^{-a-\alpha}\left(l_{1}(n)\right)^{-1} j^{a-1} X_{j} & \text { if } 1 \leq j \leq n, \\
0 & \text { if } j>n,\end{cases} \\
\mathscr{F}_{n j}= \begin{cases}\mathscr{F}_{j} & \text { if } 1 \leq j \leq n, \\
\mathscr{F}_{n} & \text { if } j>n,\end{cases}
\end{gathered}
$$

and $\phi(n)=n^{b-1} l(n)$ in Theorem 10, then $\left\{\left(X_{n j}, \mathscr{F}_{n j}\right), j \geq\right.$ $1, n \geq 1\}$ are sequences of $\mathbb{B}$-valued martingale differences. For any $n \geq 1$, we have

$$
\begin{aligned}
m_{n}(\gamma) & =\sum_{j=1}^{n} \mathbb{E}\left[\left\|n^{-a-\alpha}\left(l_{1}(n)\right)^{-1} j^{a-1} X_{j}\right\|^{\gamma} \mid \mathscr{F}_{j-1}\right] \\
& =n^{-\gamma(a+\alpha)}\left(l_{1}(n)\right)^{-\gamma} \sum_{j=1}^{n} j^{\gamma(a-1)} \mathbb{E}\left[\left\|X_{j}\right\|^{\gamma} \mid \mathscr{F}_{j-1}\right] .
\end{aligned}
$$

By (193) and Lemma 31, we have

$$
\begin{aligned}
\left\|m_{n}(\gamma)\right\|_{\mathbb{L}_{\mathbb{B}}^{q}} & \\
= & n^{-\gamma(a+\alpha)}\left(l_{1}(n)\right)^{-\gamma}\left\|\sum_{j=1}^{n} j^{\gamma(a-1)} \mathbb{E}\left[\left\|X_{j}\right\|^{\gamma} \mid \mathscr{F}_{j-1}\right]\right\|_{\mathbb{L}_{\mathbb{B}}^{q}} \\
= & n^{-\gamma(a+\alpha)}\left(l_{1}(n)\right)^{-\gamma} O\left(n^{\gamma(a-1)+1}\right) \\
= & \left(l_{1}(n)\right)^{-\gamma} O\left(n^{1-\gamma(\alpha+1)}\right) .
\end{aligned}
$$

Thus, $\left\|m_{n}(\gamma)\right\|_{\mathbb{L}_{\mathbb{B}}^{q}} \rightarrow 0$ and

$$
\begin{aligned}
& \sum_{n=1}^{\infty} n^{b-1} l(n)\left\|m_{n}(\gamma)\right\|_{\mathbb{L}_{B}^{q}}^{\lambda} \\
& \quad=\sum_{n=1}^{\infty} l(n)\left(l_{1}(n)\right)^{-\lambda \gamma} O\left(n^{b-1-\lambda(\gamma(\alpha+1)-1)}\right)<\infty
\end{aligned}
$$

whenever $\lambda \in(b /(\gamma(\alpha+1)-1), q)$. By Theorem 10, (183) is equivalent to

$$
\sum_{n=1}^{\infty} n^{b-1} l(n) \sum_{j=1}^{n} \mathbb{P}\left\{\left\|X_{j}\right\|>\varepsilon j^{-a+1} n^{a+\alpha} l_{1}(n)\right\}<\infty
$$

Notice that in view of the identically distributed assumption, (196) holds if and only if

$$
\sum_{n=1}^{\infty} n^{b-1} l(n) \sum_{j=1}^{n} \mathbb{P}\left\{\left\|X_{1}\right\|>\varepsilon j^{-a+1} n^{a+\alpha} l_{1}(n)\right\}<\infty
$$

$$
\forall \varepsilon>0 .
$$

By the monotonicity of the functions $R_{b-1}(x):=x^{b-1} l(x)$ and $R_{a+\alpha}(x)=x^{a+\alpha} l_{1}(x)$ for $x>0$ large enough and the fact that $R_{b-1}(n-1) \sim R_{b-1}(n)$ and $R_{a+\alpha}(n-1) \sim R_{a+\alpha}(n)$ as $n \rightarrow \infty$, it can be easily verified that the condition (197) is equivalent to

$$
\int_{1}^{\infty} x^{b-1} l(x)\left(\int_{1}^{x} \mathbb{P}\left\{\left\|X_{1}\right\|>\varepsilon y^{-a+1} x^{a+\alpha} l_{1}(x)\right\} \mathrm{d} y\right) \mathrm{d} x<\infty
$$

Therefore, Theorem 35 is a direct consequence of the following lemma.

Lemma 39. Let $b \geq 0, \alpha>-1, a>-\alpha$, and $l(\cdot)$ and $l_{1}(\cdot)$ are slowly varying at $\infty$. Let $X_{1}$ be any $\mathbb{B}$-valued random variable. Then, the following assertions hold.

(a) When $a \neq(b-\alpha) /(b+1)$, (198) holds if and only if (184) holds.

(b) When $a=(b-\alpha) /(b+1)$, (198) is implied by (185), where $\widetilde{l}$ is defined in (181).

Proof. We proceed as in the proof of Lemma 3.4 of Gut [20]. We distinguish three cases according to $a>1, a=1$ or $a<$ 1 . By choosing a smooth version, we can suppose that $l_{1}$ is differentiable (cf. [39]).

Case $1(a>1)$. In (198), we use the change of variables

$$
\begin{gathered}
u=y^{-a+1} x^{a+\alpha} l_{1}(x), \\
x=x .
\end{gathered}
$$

Notice that $1 \leq y \leq x$ if and only if $l_{1}(1) \leq u<\infty$ and $R_{a+\alpha}^{\leftarrow}(u) \leq x \leq R_{1+\alpha}^{\leftarrow}(u)$. Therefore, (198) holds if and only if

$$
\begin{aligned}
& \int_{l_{1}(1)}^{\infty} u^{-1 /(a-1)-1} \mathbb{P}\left\{\left\|X_{1}\right\|>\varepsilon u\right\} \\
& \quad \times\left(\int_{R_{a+\alpha}^{\leftarrow}(u)}^{R_{1+\alpha}^{\leftarrow}(u)} x^{b+(1+\alpha) /(a-1)} l(x)\left(l_{1}(x)\right)^{1 /(a-1)} \mathrm{d} x\right) \mathrm{d} u<\infty
\end{aligned}
$$


By Proposition 1.5 .8 of [39, page 26], we have as $u \rightarrow \infty$,

$$
\begin{aligned}
& \int_{U}^{R_{1+\alpha}^{\leftarrow}(u)} x^{b+(1+\alpha) /(a-1)} l(x)\left(l_{1}(x)\right)^{1 /(a-1)} \mathrm{d} x \\
& \sim\left(\left(R_{1+\alpha}^{\leftarrow}(u)\right)^{b+((1+\alpha) /(a-1))+1} l\left(R_{1+\alpha}^{\leftarrow}(u)\right)\right. \\
& \left.\quad \times\left(l_{1}\left(R_{1+\alpha}^{\leftarrow}(u)\right)\right)^{1 /(a-1)}\right) \times\left(b+\frac{1+\alpha}{a-1}+1\right)^{-1}, \\
& \int_{U}^{R_{a+\alpha}^{\leftarrow}(u)} x^{b+(1+\alpha) /(a-1)} l(x)\left(l_{1}(x)\right)^{1 /(a-1)} \mathrm{d} x \\
& \sim\left(\left(R_{a+\alpha}^{\leftarrow}(u)\right)^{b+(1+\alpha) /(a-1)+1} l\left(R_{a+\alpha}^{\leftarrow}(u)\right)\right. \\
& \left.\times\left(l_{1}\left(R_{a+\alpha}^{\leftarrow}(u)\right)\right)^{1 /(a-1)}\right) \times\left(b+\frac{1+\alpha}{a-1}+1\right)^{-1}
\end{aligned}
$$

where and hereafter $a(u) \sim b(u)$ means that $a(u) / b(u) \rightarrow 1$ as $u \rightarrow \infty$. By Lemma 34, the right hand sides of (201) are regularly varying functions of index $1 /(1+\alpha)$, and $1 /(a+\alpha)$ respectively, so that their ratio tends to $\infty$ as $u \rightarrow \infty$, since $1 /(1+\alpha)>1 /(a+\alpha)$. Therefore, as $u \rightarrow \infty$,

$$
\begin{aligned}
& \int_{R_{a+\alpha}^{\leftarrow}(u)}^{R_{1+\alpha}^{\leftarrow}(u)} x^{b+(1+\alpha) /(a-1)} l(x)\left(l_{1}(x)\right)^{1 /(a-1)} \mathrm{d} x \\
& \sim\left(\left(R_{1+\alpha}^{\leftarrow}(u)\right)^{b+(1+\alpha) /(a-1)+1} l\left(R_{1+\alpha}^{\leftarrow}(u)\right)\right. \\
& \left.\quad \times\left(l_{1}\left(R_{1+\alpha}^{\leftarrow}(u)\right)\right)^{1 /(a-1)}\right) \times\left(b+\frac{1+\alpha}{a-1}+1\right)^{-1} .
\end{aligned}
$$

Thus, (200) is equivalent to

$$
\begin{array}{r}
\int_{l_{1}(1)}^{\infty} u^{-1 /(a-1)-1}\left[R_{1+\alpha}^{\leftarrow}(u)\right]^{b+(1+\alpha) /(a-1)+1} l\left(R_{1+\alpha}^{\leftarrow}(u)\right) \\
\times\left[l_{1}\left(R_{1+\alpha}^{\leftarrow}(u)\right)\right]^{1 /(a-1)} \mathbb{P}\left\{\left\|X_{1}\right\|>\varepsilon u\right\} \mathrm{d} u<\infty \\
\forall \varepsilon>0 .
\end{array}
$$

With the change of variable $v=R_{1+\alpha}^{\leftarrow}(u)$ and the fact that $R_{1+\alpha}^{\prime}(v) \sim R_{1+\alpha}^{\prime}(v) /(1+\alpha) v$ (together with (179)), we see that (203) holds if and only if

$$
\begin{array}{r}
\int_{R_{1+\alpha}^{\leftarrow}\left(l_{1}(1)\right)}^{\infty} v^{b} l(v) \mathbb{P}\left\{R_{1+\alpha}^{\leftarrow}\left(\frac{\left\|X_{1}\right\|}{\varepsilon}\right)>v\right\} \\
\quad \mathrm{d} v<\infty \\
\forall \varepsilon>0,
\end{array}
$$

which is equivalent to the first condition of (184). Thus, (198) is equivalent to the first condition of (184).

Case $2(a=1)$. By the change of variables

$$
\begin{gathered}
u=x^{1+\alpha} l_{1}(x), \\
y=y,
\end{gathered}
$$

we see that (198) holds if and only if

$$
\begin{array}{r}
\int_{l_{1}(1)}^{\infty}\left(R_{1+\alpha}^{\leftarrow}(u)-1\right)\left(R_{1+\alpha}^{\leftarrow}(u)\right)^{b-1} l\left(R_{1+\alpha}^{\leftarrow}(u)\right) \\
\times\left(R^{\prime}\left(R_{1+\alpha}^{\leftarrow}(u)\right)\right)^{-1} \mathbb{P}\left\{\left\|X_{1}\right\|>\varepsilon u\right\} \mathrm{d} u<\infty \\
\forall \varepsilon>0 .
\end{array}
$$

With the change of variable $v=R_{1+\alpha}^{\leftarrow}(u)$, we know that (206) holds if and only if

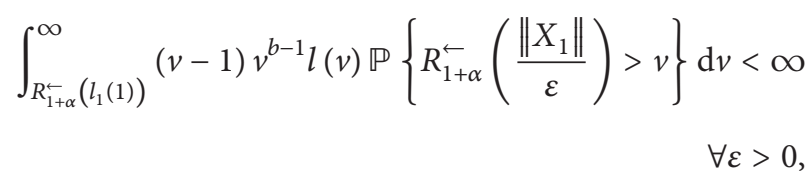

which is equivalent to the first condition of (184). Thus, (198) is equivalent to the first condition of (184).

Case $3(-\alpha<a<1)$. By the change of variables

$$
\begin{gathered}
u=y^{-a+1} x^{a+\alpha} l_{1}(x), \\
x=x,
\end{gathered}
$$

we see that (198) holds if and only if

$$
\begin{array}{r}
\int_{l_{1}(1)}^{\infty} u^{-1 /(a-1)-1} \mathbb{P}\left\{\left\|X_{1}\right\|>\varepsilon u\right\} \\
\times\left(\int_{R_{1+\alpha}^{\leftarrow}(u)}^{R_{a+\alpha}^{\leftarrow}(u)} x^{b+(1+\alpha) /(a-1)} l(x)\left(l_{1}(x)\right)^{1 /(a-1)} \mathrm{d} x\right) \mathrm{d} u<\infty \\
\forall \varepsilon>0 .
\end{array}
$$

We distinguish three cases according to $a<(b-\alpha) /(b+1)$, $a>(b-\alpha) /(b+1)$, or $a=(b-\alpha) /(b+1)$.

(i) Suppose that $a<(b-\alpha) /(b+1)$. By Proposition 1.5.8 of [39, page 26], we have as $u \rightarrow \infty$,

$$
\begin{aligned}
& \int_{U}^{R_{a+\alpha}^{\leftarrow}(u)} x^{b+(1+\alpha) /(a-1)} l(x)\left(l_{1}(x)\right)^{1 /(a-1)} \mathrm{d} x \\
& \sim\left(\left(R_{a+\alpha}^{\leftarrow}(u)\right)^{b+(1+\alpha) /(a-1)+1} l\left(R_{a+\alpha}^{\leftarrow}(u)\right)\right. \\
& \left.\quad \times\left(l_{1}\left(R_{a+\alpha}^{\leftarrow}(u)\right)\right)^{1 /(a-1)}\right) \times\left(b+\frac{1+\alpha}{a-1}+1\right)^{-1} \\
& \int_{U}^{R_{1+\alpha}^{\leftarrow}(u)} x^{b+(1+\alpha) /(a-1)} l(x)\left(l_{1}(x)\right)^{1 /(a-1)} \mathrm{d} x \\
& \sim\left(\left(R_{1+\alpha}^{\leftarrow}(u)\right)^{b+(1+\alpha) /(a-1)+1} l\left(R_{1+\alpha}^{\leftarrow}(u)\right)\right. \\
& \left.\quad \times\left(l_{1}\left(R_{1+\alpha}^{\leftarrow}(u)\right)\right)^{1 /(a-1)}\right) \times\left(b+\frac{1+\alpha}{a-1}+1\right)^{-1}
\end{aligned}
$$


so that (as in Case 1)

$$
\begin{aligned}
& \int_{R_{1+\alpha}^{\leftarrow}(u)}^{R_{a+\alpha}^{\leftarrow}(u)} x^{b+(1+\alpha) /(a-1)} l(x)\left(l_{1}(x)\right)^{1 /(a-1)} \mathrm{d} x \\
& \sim\left(\left(R_{a+\alpha}^{\leftarrow}(u)\right)^{b+(1+\alpha) /(a-1)+1} l\left(R_{a+\alpha}^{\leftarrow}(u)\right)\right. \\
& \left.\quad \times\left(l_{1}\left(R_{a+\alpha}^{\leftarrow}(u)\right)\right)^{1 /(a-1)}\right) \times\left(b+\frac{1+\alpha}{a-1}+1\right)^{-1} .
\end{aligned}
$$

Thus, (209) is equivalent to

$$
\begin{array}{r}
\int_{l_{1}(1)}^{\infty} u^{-1 /(a-1)-1}\left(R_{a+\alpha}^{\leftarrow}(u)\right)^{b+(a+\alpha) /(a-1)+1} l\left(R_{a+\alpha}^{\leftarrow}(u)\right) \\
\times\left(l_{1}\left(R_{a+\alpha}^{\leftarrow}(u)\right)\right)^{1 /(a-1)} \mathbb{P}\left\{\left\|X_{1}\right\|>\varepsilon u\right\} \mathrm{d} u<\infty \\
\forall \varepsilon>0 .
\end{array}
$$

With the change of variable $v=R_{a+\alpha}^{\leftarrow}(u)$, we see that (212) holds if and only if

$$
\int_{R_{a+\alpha}^{\leftarrow}\left(l_{1}(1)\right)}^{\infty} v^{b-1} l(v) \mathbb{P}\left\{R_{a+\alpha}^{\leftarrow}\left(\frac{\left\|X_{1}\right\|}{\varepsilon}\right)>v\right\} \mathrm{d} v<\infty \quad \forall \varepsilon>0,
$$

which is equivalent to the second condition of (184). Thus, (198) is equivalent to the second condition of (184).

(ii) Suppose that $a>(b-\alpha) /(b+1)$. By Proposition 1.5.10 of [39, page 27], we have as $u \rightarrow \infty$,

$$
\begin{aligned}
& \int_{R_{1+\alpha}^{\leftarrow}(u)}^{\infty} x^{b+(1+\alpha) /(a-1)} l(x)\left(l_{1}(x)\right)^{1 /(a-1)} \mathrm{d} x \\
& \sim\left(\left(R_{1+\alpha}^{\leftarrow}(u)\right)^{b+(1+\alpha) /(a-1)+1} l\left(R_{1+\alpha}^{\leftarrow}(u)\right)\right. \\
& \left.\quad \times\left(l_{1}\left(R_{1+\alpha}^{\leftarrow}(u)\right)\right)^{1 /(a-1)}\right) \times\left(-\left(b+\frac{1+\alpha}{a-1}+1\right)\right)^{-1} \\
& \int_{R_{a+\alpha}^{\leftarrow}(u)}^{\infty} x^{b+(1+\alpha) /(a-1)} l(x)\left(l_{1}(x)\right)^{1 /(a-1)} \mathrm{d} x \\
& \sim\left(\left(R_{a+\alpha}^{\leftarrow}(u)\right)^{b+(a+\alpha) /(a-1)+1} l\left(R_{a+\alpha}^{\leftarrow}(u)\right)\right. \\
& \left.\quad \times\left(l_{1}\left(R_{a+\alpha}^{\leftarrow}(u)\right)\right)^{1 /(a-1)}\right) \times\left(-\left(b+\frac{1+\alpha}{a-1}+1\right)\right)^{-1}
\end{aligned}
$$

so that as $u \rightarrow \infty$,

$$
\begin{aligned}
& \int_{R_{1+\alpha}^{\leftarrow}(u)}^{R_{a+\alpha}^{\leftarrow}(u)} x^{b+(1+\alpha) /(a-1)} l(x)\left(l_{1}(x)\right)^{1 /(a-1)} \mathrm{d} x \\
& \sim\left(\left(R_{1+\alpha}^{\leftarrow}(u)\right)^{b+(1+\alpha) /(a-1)+1} l\left(R_{1+\alpha}^{\leftarrow}(u)\right)\right. \\
& \left.\quad \times\left(l_{1}\left(R_{1+\alpha}^{\leftarrow}(u)\right)\right)^{1 /(a-1)}\right) \times\left(-\left(b+\frac{1+\alpha}{a-1}+1\right)\right)^{-1} .
\end{aligned}
$$

Thus, (209) holds if and only if (203) holds. Notice that (203) is equivalent to the first condition of (184). Thus (198) is equivalent to the first condition of (184). (iii) Suppose that $a=(b-\alpha) /(b+1)$. In this case, (209) reduces to

$$
\begin{aligned}
& \int_{l_{1}(1)}^{\infty} u^{-1 /(a-1)-1} \mathbb{P}\left\{\left\|X_{1}\right\|>\varepsilon u\right\} \\
& \times\left(\int_{R_{1+\alpha}^{\leftarrow}(u)}^{R_{a+\alpha}^{\leftarrow}(u)} x^{-1} l(x)\left(l_{1}(x)\right)^{1 /(a-1)} \mathrm{d} x\right) \mathrm{d} u<\infty \\
& \forall \varepsilon>0 .
\end{aligned}
$$

By Proposition 1.5.8 of [39, page 26], we have

$$
\begin{aligned}
& \int_{R_{1+\alpha}^{\leftarrow}(u)}^{R_{a+\alpha}^{\leftarrow}(u)} x^{-1} l(x)\left(l_{1}(x)\right)^{1 /(a-1)} \mathrm{d} x \\
& \quad=\widetilde{l}\left(R_{a+\alpha}^{\leftarrow}(u)\right)-\tilde{l}\left(R_{1+\alpha}^{\leftarrow}(u)\right)<\tilde{l}\left(R_{a+\alpha}^{\leftarrow}(u)\right) .
\end{aligned}
$$

So, (209) is implied by

$$
\int_{l_{1}(1)}^{\infty} u^{-1 /(a-1)-1} \widetilde{l}\left(R_{a+\alpha}^{\leftarrow}(u)\right) \mathbb{P}\left\{\left\|X_{1}\right\|>\varepsilon u\right\} \mathrm{d} u<\infty \quad \forall \varepsilon>0 .
$$

With the change of variable $v=R_{a+\alpha}^{\leftarrow}(u)$, we see that (218) holds if and only if

$$
\begin{array}{r}
\int_{R_{a+\alpha}^{\leftarrow}\left(l_{1}(1)\right)}^{\infty} v^{b-1}\left(l_{1}(v)\right)^{-1 /(a-1)} \tilde{l}(v) \\
\times \mathbb{P}\left\{R_{a+\alpha}^{\leftarrow}\left(\frac{\left\|X_{1}\right\|}{\varepsilon}\right)>v\right\} \mathrm{d} v<\infty \\
\forall \varepsilon>0,
\end{array}
$$

which is equivalent to (185). Thus, (209) is implied by (185). Therefore, (198) is implied by (185). This ends the proof of Lemma 39.

Proof of Corollary 38. We are in the case where $l_{1}(x)=$ $(\log x)^{\beta}(\beta \geq 0)$. If $y=R_{1+\alpha}(x)=x^{1+\alpha}(\log x)^{\beta}$, then

$$
\log y \sim(1+\alpha) \log x \quad \text { as } x \longrightarrow+\infty \text {. }
$$

Thus,

$$
\begin{array}{r}
x=y^{1 /(1+\alpha)}(\log x)^{-\beta /(1+\alpha)} \sim(1+\alpha)^{\beta /(1+\alpha)}\left(\frac{y}{(\log y)^{\beta}}\right)^{1 /(1+\alpha)} \\
\text { as } y \longrightarrow+\infty .
\end{array}
$$

Therefore,

$$
R_{1+\alpha}^{\leftarrow}(y) \sim(1+\alpha)^{\beta /(1+\alpha)}\left(\frac{y}{(\log y)^{\beta}}\right)^{1 /(1+\alpha)} \quad \text { as } y \longrightarrow+\infty \text {. }
$$


Similarly,

$$
R_{a+\alpha}^{\leftarrow}(y) \sim(a+\alpha)^{\beta /(a+\alpha)}\left(\frac{y}{(\log y)^{\beta}}\right)^{1 /(a+\alpha)} \text { as } y \longrightarrow+\infty .
$$

Using (222), (223), and Theorem 35, we obtain the desired results.

\section{Convergence of Weighted Sums of Banach Valued Martingale Differences of the Form $\sum_{j=1}^{\infty} a_{n j} X_{n j}$}

In this section, we consider more general weighted sums of Banach valued martingale differences than those considered in Section 7.

Let $\left(X_{j}\right)_{j \geq 1}$ be a sequence of i.i.d. random variables with $\mathbb{E} X_{j}=0$, and let $\left\{a_{n j}, j \geq 1, n \geq 1\right\}$ be an array of real numbers. The study of the convergence of weighted sums $\sum_{j=1}^{\infty} a_{n j} X_{j}$ as $n \rightarrow \infty$ is a classical subject; see for example, Salem and Zygmund [40], Hill [41], Hanson and Koopman [42], Pruitt [43], Franck and Hanson [44], Chow [45], Chow and Lai [46], and Stout [47]. Pruitt [43] found a necessary and sufficient condition for $\sum_{j=1}^{\infty} a_{n j} X_{j} \rightarrow 0$ in probability and a sufficient condition for $\sum_{j=1}^{\infty} a_{n j} X_{j} \rightarrow 0$ a.s. Baxter et al. [35] also showed a sufficient condition for $\sum_{j=1}^{\infty} a_{n j} X_{j} \rightarrow 0$ a.s. Li et al. [9] studied the complete convergence of weighted sums of independent random variables of the form $\sum_{j=1}^{\infty} a_{n j} X_{n j}$. Yu [23] and Ghosal and Chandra [19] considered the same problem for martingale differences $\left(X_{n j}\right)$. We will extend or improve some of the aforementioned works.

8.1. Law of Large Numbers for Weighted Sums of Banach Valued Martingale Differences. Let $(\mathbb{B},\|\cdot\|)$ be a separable Banach space. In this subsection, we find sufficient conditions for the convergence of weighted sums of $\mathbb{B}$-valued martingale differences $\left(X_{n j}\right)$.

Let us recall the famous theorem of Pruitt [43, Theorem 1] which states that for a sequence of i.i.d. random variables $X_{j}$ with $\mathbb{E}\left|X_{1}\right|<\infty$ and $\mathbb{E} X_{1}=0$,

$$
\sum_{j=1}^{\infty} a_{n j} X_{j} \longrightarrow 0 \quad \text { in probability }
$$

if and only if $\max _{j \geq 1}\left|a_{n j}\right| \rightarrow 0$ as $n \rightarrow \infty$, where $\left\{a_{n j}, j \geq 1, n \geq 1\right\}$ is a Toeplitz summation matrix; that is, $\lim _{n \rightarrow \infty} a_{n j}=0$ for every $j \geq 1, \lim _{n \rightarrow \infty} \sum_{j=1}^{\infty} a_{n j}=1$, and $\sup _{n \geq 1} \sum_{j=1}^{\infty}\left|a_{n j}\right|<\infty$.

In the following, we consider the same problem for $\mathbb{B}$ valued martingale differences $\left(X_{n j}\right)$.
Theorem 40. Let $\left\{\left(X_{n j}, \mathscr{F}_{n j}\right)\right\}_{j \geq 1}, n \geq 1$, be sequences of $\mathbb{B}$ valued martingale differences. Let $\left\{a_{n j}, j \geq 1, n \geq 1\right\}$ be an array of real numbers satisfying $\sup _{n \geq 1} \sum_{j=1}^{\infty}\left|a_{n j}\right|<\infty$ and $A_{n}=\sup _{j \geq 1}\left|a_{n j}\right| \rightarrow 0$ as $n \rightarrow \infty$. Suppose that for some $\gamma \in(1,2]$, there exists $K>0$, such that

$$
\mathbb{E}\left\|X_{n j}\right\|^{\gamma} \leq K
$$

If $\mathbb{B}$ is $\gamma$-smooth, then

$$
\sum_{j=1}^{\infty} a_{n j} X_{n j} \longrightarrow 0 \quad \text { in probability. }
$$

Proof. Since

$$
\begin{aligned}
\mathbb{E} m_{n}(\gamma) & =\sum_{j=1}^{\infty} \mathbb{E}\left\|a_{n j} X_{n j}\right\|^{\gamma} \leq \sum_{j=1}^{\infty}\left|a_{n j}\right| A_{n}^{\gamma-1} \mathbb{E}\left\|X_{n j}\right\|^{\gamma} \\
& \leq A_{n}^{\gamma-1} K \sum_{j=1}^{\infty}\left|a_{n j}\right| \longrightarrow 0
\end{aligned}
$$

we see that (226) follows from Theorem 4.

Let $\left\{a_{n j}, j \geq 1, n \geq 1\right\}$ be a Toeplitz summation matrix; Theorem 2 of Pruitt [43] states that for a sequence $\left(X_{j}\right)_{j \geq 1}$ of i.i.d. random variables with $\mathbb{E}\left|X_{1}\right|<\infty$ and $\mathbb{E} X_{1}=0$, if $\max _{j \geq 1}\left|a_{n j}\right|=O\left(n^{-\beta}\right), \beta>0$, then $\mathbb{E}\left|X_{1}\right|^{1+1 / \beta}<\infty$ implies that

$$
\sum_{j=1}^{\infty} a_{n j} X_{j} \longrightarrow 0 \quad \text { a.s. }
$$

In the following, we also consider the similar problem for arrays of $\mathbb{B}$-valued martingale differences $\left(X_{n j}\right)$.

Theorem 41. Let $\left\{\left(X_{n j}, \mathscr{F}_{n j}\right)\right\}_{j \geq 1}, n \geq 1$, be sequences of $\mathbb{B}$ valued martingale differences. Let $\left\{a_{n j}, j \geq 1, n \geq 1\right\}$ be an array of real numbers satisfying $\sup _{n \geq 1} \sum_{j=1}^{\infty}\left|a_{n j}\right|<\infty$ and $\max _{j \geq 1}\left|a_{n j}\right|=O\left(n^{-\beta}\right), \beta>0$. Suppose that, for some $\gamma \in$ $(1,2]$, there exists a constant $K>0$ such that $\mathbb{E}\left\|X_{n j}\right\|^{\gamma} \leq K$ for all $j \geq 1, n \geq 1$. If $\mathbb{B}$ is $\gamma$-smooth, then

$$
\mathbb{P}\left\{\left\|\sum_{j=1}^{\infty} a_{n j} X_{n j}\right\|>\varepsilon\right\}=O\left(n^{-\beta(\gamma-1)}\right) \quad \forall \varepsilon>0
$$

Consequently, if $\mathbb{B}$ is $\gamma$-smooth and $\sup _{n, j \geq 1} \mathbb{E}\left\|X_{n j}\right\|^{\gamma}<\infty$ for some $\gamma>1+1 / \beta$, then

$$
\sum_{j=1}^{\infty} a_{n j} X_{n j} \longrightarrow 0 \text { a.s. }
$$


Proof. Set $Y_{n j}=a_{n j} X_{n j}, j \geq 1, n \geq 1$, then $\left\{\left(Y_{n j}, \mathscr{F}_{n j}\right), j \geq\right.$ $1, n \geq 1\}$ is an array of $\mathbb{B}$-valued martingale differences and for any $n \geq 1$,

$$
\begin{aligned}
\mathbb{E} m_{n}(\gamma) & =\mathbb{E}\left[\sum_{j=1}^{\infty} \mathbb{E}\left[\left\|Y_{n j}\right\|^{\gamma} \mid \mathscr{F}_{n, j-1}\right]\right] \\
& =\sum_{j=1}^{\infty} \mathbb{E}\left\|Y_{n j}\right\|^{\gamma}=\sum_{j=1}^{\infty}\left|a_{n j}\right|^{\gamma} \mathbb{E}\left\|X_{n j}\right\|^{\gamma} \\
& \leq\left(\sup _{n \geq 1} \sum_{j=1}^{\infty}\left|a_{n j}\right|\right)\left(C n^{-\beta}\right)^{\gamma-1} K \\
& \leq C_{1}(\gamma) n^{-\beta(\gamma-1)} .
\end{aligned}
$$

By Corollary 3, we see that

$$
\mathbb{P}\left\{\left\|\sum_{j=1}^{\infty} a_{n j} X_{n j}\right\|>\varepsilon\right\} \leq \mathbb{P}\left\{\sup _{j \geq 1}\left\|\sum_{i=1}^{j} a_{n j} X_{n j}\right\|>\varepsilon\right\} .
$$

By Theorem 2 with $q=1$ and $L=0$, we know that

$$
\begin{aligned}
\mathbb{P} & \left\{\sup _{j \geq 1}\left\|\sum_{i=1}^{j} a_{n j} X_{n j}\right\|>\varepsilon\right\} \\
& \leq \mathbb{P}\left\{\sup _{j \geq 1}\left\|a_{n j} X_{n j}\right\|>\frac{\varepsilon}{4}\right\}+\varepsilon^{-\gamma} C_{2}(\gamma) \mathbb{E} m_{n}(\gamma) .
\end{aligned}
$$

By Theorem 1, we see that

$$
\mathbb{P}\left\{\sup _{j \geq 1}\left\|a_{n j} X_{n j}\right\|>\frac{\varepsilon}{4}\right\} \leq \sum_{j=1}^{\infty} \mathbb{P}\left\{\left\|a_{n j} X_{n j}\right\|>\frac{\varepsilon}{4}\right\} .
$$

Since (231), we know that

$$
\begin{aligned}
\sum_{j=1}^{\infty} \mathbb{P}\left\{\left\|a_{n j} X_{n j}\right\|>\frac{\varepsilon}{4}\right\} & \leq 4^{\gamma} \varepsilon^{-\gamma} \sum_{j=1}^{\infty}\left|a_{n j}\right|^{\gamma} \mathbb{E}\left\|X_{n j}\right\|^{\gamma} \\
& \leq 4^{\gamma} \varepsilon^{-\gamma} C_{1}(\gamma) n^{-\beta(\gamma-1)} .
\end{aligned}
$$

Since (231)-(235), we have

$$
\begin{aligned}
\mathbb{P}\left\{\left\|\sum_{j=1}^{\infty} a_{n j} X_{n j}\right\|>\varepsilon\right\} \leq 4^{\gamma} \varepsilon^{-\gamma} C_{1}(\gamma) n^{-\beta(\gamma-1)} \\
+\varepsilon^{-\gamma} C_{2}(\gamma) C_{1}(\gamma) n^{-\beta(\gamma-1)} .
\end{aligned}
$$

Thus, (229) holds.

If additionally $\gamma>1+1 / \beta$, then (229) implies that

$$
\sum_{n=1}^{\infty} \mathbb{P}\left\{\left\|\sum_{j=1}^{\infty} a_{n j} X_{n j}\right\|>\varepsilon\right\}<\infty \quad \forall \varepsilon>0 .
$$

Therefore, (230) holds.
The following theorem extends Theorem 3.3 of Baxter et al. [35].

Theorem 42. Let $\left\{\left(X_{j}, \mathscr{F}_{j}\right)\right\}_{j \geq 1}$ be $\mathbb{B}$-valued martingale differences. Suppose that for some $\gamma \in(1,2]$, there exists $K>0$, such that for all $j \geq 1$,

$$
\mathbb{E}\left\|X_{j}\right\|^{\gamma} \leq K .
$$

Let $\left\{a_{j}, j \geq 1\right\}$ satisfy

$$
\frac{1}{n} \sum_{j=1}^{n}\left|a_{j}\right|^{\gamma}=O\left(n^{\delta}\right) \quad \text { for some } \delta \in \mathbb{R}
$$

and set $T_{n}=\sum_{j=1}^{n} a_{j} X_{j}$. If $\mathbb{B}$ is $\gamma$-smooth, then

$$
\begin{gathered}
\frac{T_{n}}{n^{\alpha}} \longrightarrow 0 \text { a.s. for } \alpha>\frac{\delta}{\gamma}, \\
\mathbb{P}\left\{\sup _{m \geq n} \frac{\left\|T_{m}\right\|}{m^{\alpha}}>\varepsilon\right\}=O\left(n^{-(\gamma \alpha-\delta)}\right) .
\end{gathered}
$$

When $\left(X_{j}, j \geq 1\right)$ are i.i.d. real-valued random variables, $\alpha \leq 1$, and $\delta=1,(240)$ reduces to Theorem 3.3 of Baxter et al. [35].

Proof of Theorem 42. Clearly,

$$
\mathbb{P}\left\{\sup _{m \geq n} \frac{\left\|T_{m}\right\|}{m^{\alpha}}>\varepsilon\right\} \leq \mathbb{P}\left\{\sup _{m \geq n}\left(m^{-\alpha} \max _{1 \leq j \leq m}\left\|T_{j}\right\|\right)>\varepsilon\right\} .
$$

By Theorem 22, we see that

$$
\mathbb{P}\left\{\sup _{m \geq n}\left(m^{-\alpha} \max _{1 \leq j \leq m}\left\|T_{j}\right\|\right)>\varepsilon\right\}=O\left(n^{-(\gamma \alpha-\delta)}\right) \quad \forall \varepsilon>0
$$

if and only if

$$
\mathbb{P}\left\{\max _{1 \leq j \leq n}\left\|T_{j}\right\|>\varepsilon n^{\alpha}\right\}=O\left(n^{-(\gamma \alpha-\delta)}\right) \quad \forall \varepsilon>0 .
$$

By (238) and (239), we have

$$
\mathbb{E} m(\gamma, n)=\sum_{j=1}^{n} \mathbb{E}\left\|a_{j} X_{j}\right\|^{\gamma}=\sum_{j=1}^{n}\left|a_{j}\right|^{\gamma} \mathbb{E}\left\|X_{j}\right\|^{\gamma} \leq C K n^{\delta} .
$$

And by Theorem 2 with $q=1$ and $L=0$, we know that

$$
\begin{aligned}
\mathbb{P} & \left\{\max _{1 \leq j \leq n}\left\|T_{j}\right\|>\varepsilon n^{\alpha}\right\} \\
& \leq \mathbb{P}\left\{\max _{1 \leq j \leq n}\left\|a_{j} X_{j}\right\|>\frac{1}{4} \varepsilon n^{\alpha}\right\}+\left(\varepsilon n^{\alpha}\right)^{-\gamma} C_{1}(\gamma) \mathbb{E} m(\gamma, n) \\
& \leq \mathbb{P}\left\{\max _{1 \leq j \leq n}\left\|a_{j} X_{j}\right\|>\frac{1}{4} \varepsilon n^{\alpha}\right\}+C K C_{1}(\gamma) \varepsilon^{-\gamma} n^{-(\gamma \alpha-\delta)} .
\end{aligned}
$$


By Theorem 1, we see that

$$
\mathbb{P}\left\{\max _{1 \leq j \leq n}\left\|a_{j} X_{j}\right\|>\frac{1}{4} \varepsilon n^{\alpha}\right\} \leq \sum_{j=1}^{n} \mathbb{P}\left\{\left\|a_{j} X_{j}\right\|>\frac{1}{4} \varepsilon n^{\alpha}\right\} .
$$

By (238) and (239), we know that

$$
\begin{aligned}
& \sum_{j=1}^{n} \mathbb{P}\left\{\left\|a_{j} X_{j}\right\|>\frac{1}{4} \varepsilon n^{\alpha}\right\} \\
& \leq 4^{\gamma} \varepsilon^{-\gamma} n^{-\gamma \alpha} \sum_{j=1}^{n}\left|a_{j}\right|^{\gamma} \mathbb{E}\left\|X_{j}\right\|^{\gamma} \\
& \leq 4^{\gamma} C K \varepsilon^{-\gamma} n^{-(\gamma \alpha-\delta)} .
\end{aligned}
$$

From (246)-(248), we see that (244) holds. Thus, (241) holds.

8.2. Complete Convergence of Weighted Sums of Banach Valued Martingale Differences. Let $(\mathbb{B},\|\cdot\|)$ be a separable Banach space. In this subsection, we consider complete convergence of weighted sums of $\mathbb{B}$-valued martingale differences $\left(X_{n j}\right)$ of the form $\sum_{j=1}^{\infty} a_{n j} X_{n j}$. We extend and improve Corollary 1 of Ghosal and Chandra [19] and Theorems 2.2-2.4 of Li et al. [9]. We also generalize Theorem 2 of Yu [23].

Theorem 43. Let $\left\{\left(X_{n j}, \mathscr{F}_{n j}\right)\right\}_{j \geq 1}, n \geq 1$, be sequences of $\mathbb{B}$ valued martingale differences. Let $\alpha>0$ and $\left\{a_{n j}, j \geq 1, n \geq 1\right\}$ be an array of real numbers. If there exists $\gamma \in(1,2]$ such that

$$
\sum_{n=1}^{\infty} \sum_{j=1}^{\infty} n^{-\gamma \alpha}\left|a_{n j}\right|^{\gamma} \mathbb{E}\left\|X_{n j}\right\|^{\gamma}<\infty
$$

and $\mathbb{B}$ is $\gamma$-smooth, then

$$
\sum_{n=1}^{\infty} \mathbb{P}\left\{n^{-\alpha} \sup _{j \geq 1}\left\|\sum_{i=1}^{j} a_{n i} X_{n i}\right\|>\varepsilon\right\}<\infty \quad \forall \varepsilon>0 .
$$

In the square-integrable real-valued martingale differences case, the result was proved by Ghosal and Chandra in Corollary 1 [19] if, additionally, $\sum_{j=1}^{\infty} a_{n j}^{2}=O\left(n^{\delta}\right)$ for some $\delta<2 \alpha$.

We generalize Theorem 2 of $\mathrm{Yu}$ [23] from two directions by Theorem 43: first, we extend sequences of $L^{p}(p \geq 2)$ martingale differences to $L^{\gamma}(\gamma \in(1,2])$ sequences of $\mathbb{B}$ valued martingale differences; secondly, we do not need the condition $\sum_{j=1}^{\infty}\left|a_{n j}\right|^{2}=O\left(n^{\delta}\right)$ for some $\delta<\alpha$.

Proof of Theorem 43. Set $Y_{n j}=n^{-\alpha} a_{n j} X_{n j}$. From (249), we have

$$
\begin{aligned}
\sum_{n=1}^{\infty} \mathbb{E} m_{n}(\gamma) & =\sum_{n=1}^{\infty} \sum_{j=1}^{\infty} \mathbb{E}\left\|Y_{n j}\right\|^{\gamma} \\
& =\sum_{n=1}^{\infty} \sum_{j=1}^{\infty} n^{-\gamma \alpha}\left|a_{n j}\right|^{\gamma} \mathbb{E}\left\|X_{n j}\right\|^{\gamma}<\infty .
\end{aligned}
$$

By Corollary 3 and Theorem 2 with $q=1$ and $L=0$, we see that

$$
\begin{aligned}
& \mathbb{P}\left\{n^{-\alpha} \sup _{j \geq 1}\left\|\sum_{i=1}^{j} a_{n i} X_{n i}\right\|>\varepsilon\right\} \\
& \leq \mathbb{P}\left\{n^{-\alpha} \sup _{j \geq 1}\left\|a_{n j} X_{n j}\right\|>\frac{\varepsilon}{4}\right\}+\varepsilon^{-\gamma} C_{2}(\gamma) \mathbb{E} m_{n}(\gamma) .
\end{aligned}
$$

By Corollary 3 and Theorem 1, we know that

$$
\mathbb{P}\left\{n^{-\alpha} \sup _{j \geq 1}\left\|a_{n j} X_{n j}\right\|>\frac{\varepsilon}{4}\right\} \leq \sum_{j=1}^{\infty} \mathbb{P}\left\{n^{-\alpha}\left\|a_{n j} X_{n j}\right\|>\frac{\varepsilon}{4}\right\} .
$$

By Markov's inequality, we see that

$$
\sum_{j=1}^{\infty} \mathbb{P}\left\{n^{-\alpha}\left\|a_{n j} X_{n j}\right\|>\frac{\varepsilon}{4}\right\} \leq 4^{\gamma} \varepsilon^{-\gamma} \sum_{j=1}^{\infty} n^{-\gamma \alpha}\left|a_{n j}\right|^{\gamma} \mathbb{E}\left\|X_{n j}\right\|^{\gamma} .
$$

Since (249) and (251)-(254), we see that (250) holds.

Theorem 44. Let $\left\{\left(X_{n j}, \mathscr{F}_{n j}\right)\right\}_{j \geq 1}, n \geq 1$, be sequences of $\mathbb{B}$ valued martingale differences. Let $\left\{a_{n j}, j \geq 1, n \geq 1\right\}$ be an array of real numbers. Suppose that for some constants $\gamma \in$ $(1,2], K>0, \alpha \in(0,1 / 2]$ and $\delta<\gamma \alpha$,

$$
\begin{gathered}
\sum_{j=1}^{\infty}\left|a_{n j}\right|^{\gamma} \leq K n^{\delta}, \quad n \geq 1, \\
\sum_{n=1}^{\infty} n^{-1} \sum_{j=1}^{\infty}\left|a_{n j}\right|^{1 / \alpha}<\infty \\
\sup _{n, j \geq 1} \mathbb{E}\left\|X_{n j}\right\|^{1 / \alpha}<\infty .
\end{gathered}
$$

If $\mathbb{B}$ is $\gamma$-smooth, then (250) holds.

When $\left(X_{n j}\right)_{j \geq 1}, n \geq 1$, is a sequence of zero mean independent real-valued random variables and $\gamma=2$, Theorem 44 reduces to Theorem 2.3 of Li et al. [9].

Proof of Theorem 44. Set $Y_{n j}=n^{-\alpha} a_{n j} X_{n j}, j \geq 1, n \geq 1$, then $\left\{\left(Y_{n j}, \mathscr{F}_{n j}\right), j \geq 1, n \geq 1\right\}$ is an array of $\mathbb{B}$-valued martingale differences. Since (255) and (257), for any $n \geq 1$, we have

$$
\begin{aligned}
\sum_{n=1}^{\infty} \mathbb{E} m_{n}(\gamma) & =\sum_{n=1}^{\infty} \sum_{j=1}^{\infty} \mathbb{E}\left\|n^{-\alpha} a_{n j} X_{n j}\right\|^{\gamma} \\
& =\sum_{n=1}^{\infty} n^{-\gamma \alpha} \sum_{j=1}^{\infty}\left|a_{n j}\right|^{\gamma} \mathbb{E}\left\|X_{n j}\right\|^{\gamma} \\
& \leq C K \sum_{n=1}^{\infty} n^{-(\gamma \alpha-\delta)}<\infty .
\end{aligned}
$$


By Corollary 3 and Theorem 2 with $q=1$ and $L=0$, we see that

$$
\begin{aligned}
& \mathbb{P}\left\{n^{-\alpha} \sup _{j \geq 1}\left\|\sum_{i=1}^{j} a_{n j} X_{n j}\right\|>\varepsilon\right\} \\
& \leq \mathbb{P}\left\{n^{-\alpha} \sup _{j \geq 1}\left\|a_{n j} X_{n j}\right\|>\frac{\varepsilon}{4}\right\}+\varepsilon^{-\gamma} C_{2}(\gamma) \mathbb{E} m_{n}(\gamma) .
\end{aligned}
$$

By Corollary 3 and Theorem 1, we know that

$$
\mathbb{P}\left\{n^{-\alpha} \sup _{j \geq 1}\left\|a_{n j} X_{n j}\right\|>\frac{\varepsilon}{4}\right\} \leq \sum_{j=1}^{\infty} \mathbb{P}\left\{n^{-\alpha}\left\|a_{n j} X_{n j}\right\|>\frac{\varepsilon}{4}\right\} .
$$

By Markov's inequality, we see that

$$
\begin{aligned}
& \sum_{j=1}^{\infty} \mathbb{P}\left\{n^{-\alpha}\left\|a_{n j} X_{n j}\right\|>\frac{\varepsilon}{4}\right\} \\
& \leq \varepsilon^{-1 / \alpha} n^{-1} \sum_{j=1}^{\infty}\left|a_{n j}\right|^{1 / \alpha} \mathbb{E}\left\|X_{n j}\right\|^{1 / \alpha}<\infty .
\end{aligned}
$$

By (256), (257), and (258)-(261), we know that (250) holds.

Theorem 45. Let $\left\{\left(X_{n j}, \mathscr{F}_{n j}\right)\right\}_{j=1}^{n}$ be a triangular array of identically distributed $\mathbb{B}$-valued martingale differences. Let $\beta>$ -1 , and let $\left\{a_{n j}, 1 \leq j \leq n\right\}$ be a triangular array of positive numbers satisfying

$$
a_{n j}=c_{n j} n^{-\beta-1} j^{\beta}
$$

with $0<c \leq c_{n j} \leq C<\infty$ for every $1 \leq j \leq n, n \geq 1$ and some constants $c$ and $C$. Suppose that there exists some constant $K$, such that, for some $\gamma \in(1,2]$,

$$
\sup _{n, j \geq 1} \mathbb{E}\left[\left\|X_{n j}\right\|^{\gamma} \mid \mathscr{F}_{n, j-1}\right] \leq K
$$

Suppose that

$$
\begin{gathered}
\mathbb{E}\left\|X_{11}\right\|^{1 /(1+\beta)}<\infty \quad \text { if }-1<\beta<-\frac{1}{2}, \\
\mathbb{E}\left\|X_{11}\right\|^{2} \log \left(1+\left\|X_{11}\right\|\right)<\infty \quad \text { if } \beta=-\frac{1}{2}, \\
\mathbb{E}\left\|X_{11}\right\|^{2}<\infty \quad \text { if } \beta>-\frac{1}{2},
\end{gathered}
$$

then for all $\varepsilon>0$,

$$
\sum_{n=1}^{\infty} \mathbb{P}\left\{\left\|\sum_{j=1}^{n} a_{n j} X_{n j}\right\|>\varepsilon\right\}<\infty
$$

When $\left\{X_{n j}\right\}=\left\{X_{j}\right\}$ are the same sequence of i.i.d. realvalued random variables and $\sum_{j=1}^{n} a_{n j}=1$ for all $n \geq 1$, Theorem 45 reduces to the sufficiency of Theorem 2.4 of $\mathrm{Li}$ et al. [9].
Proof of Theorem 45. Set

$$
Y_{n j}= \begin{cases}a_{n j} X_{n j} & \text { if } 1 \leq j \leq n \\ 0 & \text { if } j>n\end{cases}
$$

and $c_{n}=1$, then $\left\{\left(Y_{n j}, \mathscr{F}_{n j}\right)\right\}_{j=1}^{n}$ are sequences of $\mathbb{B}$-valued martingale differences. For any $n \geq 1$, since (262) and (263), we have

$$
\begin{aligned}
m_{n}(\gamma) & =\sum_{j=1}^{n} \mathbb{E}\left[\left\|a_{n j} X_{n j}\right\|^{\gamma} \mid \mathscr{F}_{n, j-1}\right] \\
& =\sum_{j=1}^{n} a_{n j}^{\gamma} \mathbb{E}\left[\left\|X_{n j}\right\|^{\gamma} \mid \mathscr{F}_{n, j-1}\right] \\
& \leq K C^{\gamma} n^{-(\gamma-1)}\left(n^{-1} \sum_{j=1}^{n}\left(\frac{j}{n}\right)^{\gamma \beta}\right) \\
& \sim K C^{\gamma} n^{-(\gamma-1)} \int_{1 / n}^{1} x^{\gamma \beta} \mathrm{d} x \\
& =O\left(n^{-b}\right),
\end{aligned}
$$

where $b=\min \{\gamma(\beta+1), \gamma-1\}$. By Corollary 12 of Theorem 10, (265) is implied by

$$
\sum_{n=1}^{\infty} \sum_{j=1}^{n} \mathbb{P}\left\{\left\|a_{n j} X_{n j}\right\|>\varepsilon\right\}<\infty \quad \forall \varepsilon>0 .
$$

Equation (268) holds if and only if (264) holds. (cf. [9, pages 62-63])

\section{Acknowledgment}

The author is most grateful to editor Dumitru Motreanu and an anonymous referee for their careful reading and insightful comments. This work has been partially supported by the Research Fund of Beijing International Studies University (no. 13Bb023) and Doctoral Research Start-up Funds Projects of Beijing International Studies University.

\section{References}

[1] P. L. Hsu and H. Robbins, "Complete convergence and the law of large numbers," Proceedings of the National Academy of Sciences of the United States of America, vol. 33, pp. 25-31, 1947.

[2] P. Erdös, "On a theorem of Hsu and Robbins," Annals of Mathematical Statistics, vol. 20, pp. 286-291, 1949.

[3] P. Erdös, "Remark on my paper 'On a theorem of Hsu and Robbin," Annals of Mathematical Statistics, vol. 21, article 138, 1950.

[4] F. Spitzer, "A combinatorial lemma and its application to probability theory," Transactions of the American Mathematical Society, vol. 82, pp. 323-339, 1956.

[5] M. L. Katz, "The probability in the tail of a distribution," Annals of Mathematical Statistics, vol. 34, pp. 312-318, 1963.

[6] L. E. Baum and M. Katz, "Convergence rates in the law of large numbers," Transactions of the American Mathematical Society, vol. 120 , pp. 108-123, 1965. 
[7] T. L. Lai, "Limit theorems for delayed sums," Annals of Probability, vol. 2, pp. 432-440, 1974.

[8] M. U. Gafurov and A. D. Slastnikov, "Some problems on the exit of a random walk from a curvilinear boundary, and large deviations," Theory of Probability and Its Applications, vol. 32, no. 2, pp. 327-348, 1987.

[9] D. L. Li, M. B. Rao, T. F. Jiang, and X. C. Wang, "Complete convergence and almost sure convergence of weighted sums of random variables," Journal of Theoretical Probability, vol. 8, no. 1, pp. 49-76, 1995.

[10] T.-C. Hu, D. Szynal, and A. I. Volodin, "A note on complete convergence for arrays," Statistics \& Probability Letters, vol. 38, no. 1, pp. 27-31, 1998.

[11] T.-C. Hu and A. Volodin, "Addendum to: 'A note on complete convergence for arrays", Statistics \& Probability Letters, vol. 38, no. 1, pp. 27-31, 1998.

[12] T.-C. Hu, M. Ordóñez Cabrera, S. H. Sung, and A. Volodin, "Complete convergence for arrays of rowwise independent random variables," Korean Mathematical Society, vol. 18, no. 2, pp. 375-383, 2003.

[13] A. Kuczmaszewska, "On some conditions for complete convergence for arrays," Statistics \& Probability Letters, vol. 66, no. 4, pp. 399-405, 2004.

[14] S. H. Sung, A. I. Volodin, and T.-C. Hu, "More on complete convergence for arrays," Statistics \& Probability Letters, vol. 71, no. 4, pp. 303-311, 2005.

[15] V. M. Kruglov, A. I. Volodin, and T.-C. Hu, "On complete convergence for arrays," Statistics \& Probability Letters, vol. 76, no. 15, pp. 1631-1640, 2006.

[16] E. Lesigne and D. Volný, "Large deviations for martingales," Stochastic Processes and their Applications, vol. 96, no. 1, pp. 143159, 2001.

[17] G. Stoica, "Baum-Katz-Nagaev type results for martingales," Journal of Mathematical Analysis and Applications, vol. 336, no. 2, pp. 1489-1492, 2007.

[18] G. Alsmeyer, "Convergence rates in the law of large numbers for martingales," Stochastic Processes and their Applications, vol. 36, no. 2, pp. 181-194, 1990.

[19] S. Ghosal and T. K. Chandra, "Complete convergence of martingale arrays," Journal of Theoretical Probability, vol. 11, no. 3, pp. 621-631, 1998.

[20] A. Gut, "Complete convergence and Cesàro summation for i.i.d. random variables," Probability Theory and Related Fields, vol. 97, no. 1-2, pp. 169-178, 1993.

[21] H. Lanzinger and U. Stadtmüller, "Baum-Katz laws for certain weighted sums of independent and identically distributed random variables," Bernoulli, vol. 9, no. 6, pp. 985-1002, 2003.

[22] Y. Wang, X. Liu, and C. Su, "Equivalent conditions of complete convergence for independent weighted sums," Science in China A, vol. 41, no. 9, pp. 939-949, 1998.

[23] K. F. Yu, "Complete convergence of weighted sums of martingale differences," Journal of Theoretical Probability, vol. 3, no. 2, pp. 339-347, 1990.

[24] D. L. Li, M. B. Rao, and X. C. Wang, "Complete convergence of moving average processes," Statistics \& Probability Letters, vol. 14, no. 2, pp. 111-114, 1992.

[25] Q. M. Shao, "Complete convergence for $\alpha$-mixing sequences," Statistics \& Probability Letters, vol. 16, no. 4, pp. 279-287, 1993.

[26] Q. M. Shao, "Maximal inequalities for partial sums of $\rho$-mixing sequences," Annals of Probability, vol. 23, no. 2, pp. 948-965, 1995.
[27] Z. Szewczak, “On Marcinkiewicz-Zygmund laws," Journal of Mathematical Analysis and Applications, vol. 375, no. 2, pp. 738744, 2011.

[28] J.-I. Baek and S.-T. Park, "Convergence of weighted sums for arrays of negatively dependent random variables and its applications," Journal of Theoretical Probability, vol. 23, no. 2, pp. 362-377, 2010.

[29] H.-Y. Liang, "Complete convergence for weighted sums of negatively associated random variables," Statistics \& Probability Letters, vol. 48, no. 4, pp. 317-325, 2000.

[30] H.-Y. Liang and C. Su, "Complete convergence for weighted sums of NA sequences," Statistics \& Probability Letters, vol. 45, no. 1, pp. 85-95, 1999.

[31] A. Kuczmaszewska, "On complete convergence in Marcinkiewicz-Zygmund type SLLN for negatively associated random variables," Acta Mathematica Hungarica, vol. 128, no. 1-2, pp. 116-130, 2010.

[32] V. M. Kruglov, "Complete convergence for maximal sums of negatively associated random variables," Journal of Probability and Statistics, vol. 2010, Article ID 764043, 17 pages, 2010.

[33] M.-H. Ko, "On the complete convergence for negatively associated random fields," Taiwanese Journal of Mathematics, vol. 15, no. 1, pp. 171-179, 2011.

[34] Y. S. Chow and H. Teicher, Probability Theory: Independent, Interchangeability, Martingales, Springer, New York, NY, USA, 3rd edition, 1997.

[35] J. Baxter, R. Jones, M. Lin, and J. Olsen, "SLLN for weighted independent identically distributed random variables," Journal of Theoretical Probability, vol. 17, no. 1, pp. 165-181, 2004.

[36] G. Pisier, "Martingales with values in uniformly convex spaces," Israel Journal of Mathematics, vol. 20, no. 3-4, pp. 326-350, 1975.

[37] N. V. Huan and N. V. Quang, "The Doob inequality and strong law of large numbers for multidimensional arrays in general Banach spaces," Kybernetika, vol. 48, no. 2, pp. 254-267, 2012.

[38] P. Assouad, "Espaces $p$-lisses et $q$-convexes, inégalités de Burkholder," in Proceedings of the Séminaire Maurey-Schwartz 1974-1975, Espaces L ${ }^{p}$, Applications Radonifiantes et Géométrie des Espaces de Banach, no. 15, p. 8, Centre de Mathématiques Appliquées-École Polytechnique, 1975.

[39] N. H. Bingham, C. M. Goldie, and J. L. Teugels, Regular Variation, vol. 27, Cambridge University Press, Cambridge, UK, 1987.

[40] R. Salem and A. Zygmund, "Some properties of trigonometric series whose terms have random signs," Acta Mathematica, vol. 91, pp. 245-301, 1954.

[41] J. D. Hill, “The Borel property of summability methods," Pacific Journal of Mathematics, vol. 1, pp. 399-409, 1951.

[42] D. L. Hanson and L. H. Koopmans, "On the convergence rate of the law of large numbers for linear combinations of independent random variables," Annals of Mathematical Statistics, vol. 36, pp. 559-564, 1965.

[43] W. E. Pruitt, "Summability of independent random variables," Journal of Applied Mathematics and Mechanics, vol. 15, pp. 769776, 1966.

[44] W. E. Franck and D. L. Hanson, "Some results giving rates of convergence in the law of large numbers for weighted sums of independent random variables," Bulletin of the American Mathematical Society, vol. 72, pp. 266-268, 1966.

[45] Y. S. Chow, "Some convergence theorems for independent random variables," Annals of Mathematical Statistics, vol. 37, pp. 1482-1493, 1966. 
[46] Y. S. Chow and T. L. Lai, "Limiting behavior of weighted sums of independent random variables," Annals of Probability, vol. 1, pp. 810-824, 1973.

[47] W. F. Stout, "Some results on the complete and almost sure convergence of linear combinations of independent random variables and martingale differences," Annals of Mathematical Statistics, vol. 39, pp. 1549-1562, 1968. 


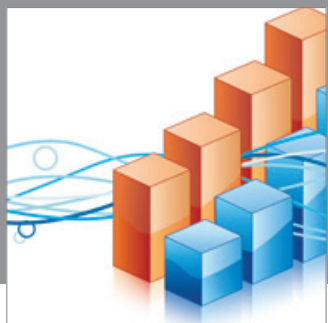

Advances in

Operations Research

mansans

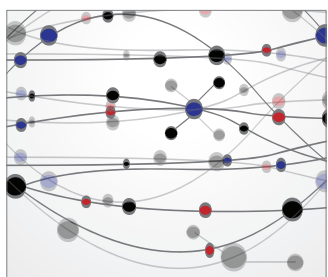

The Scientific World Journal
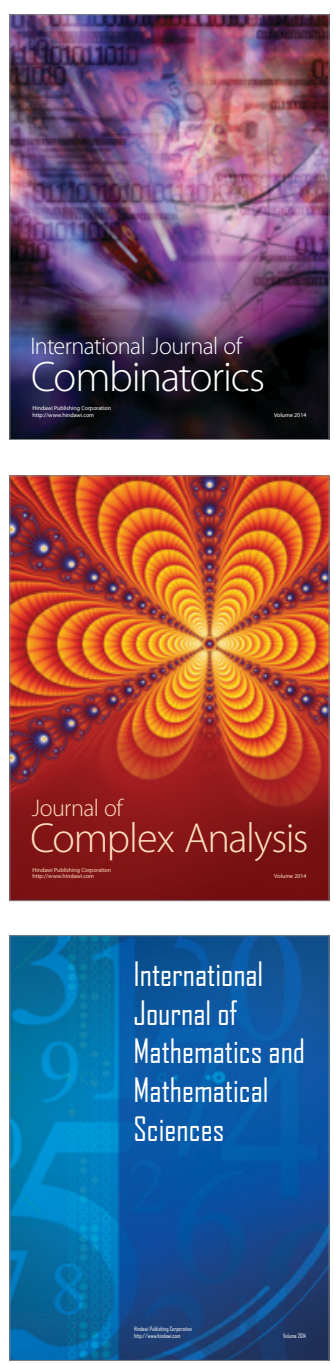
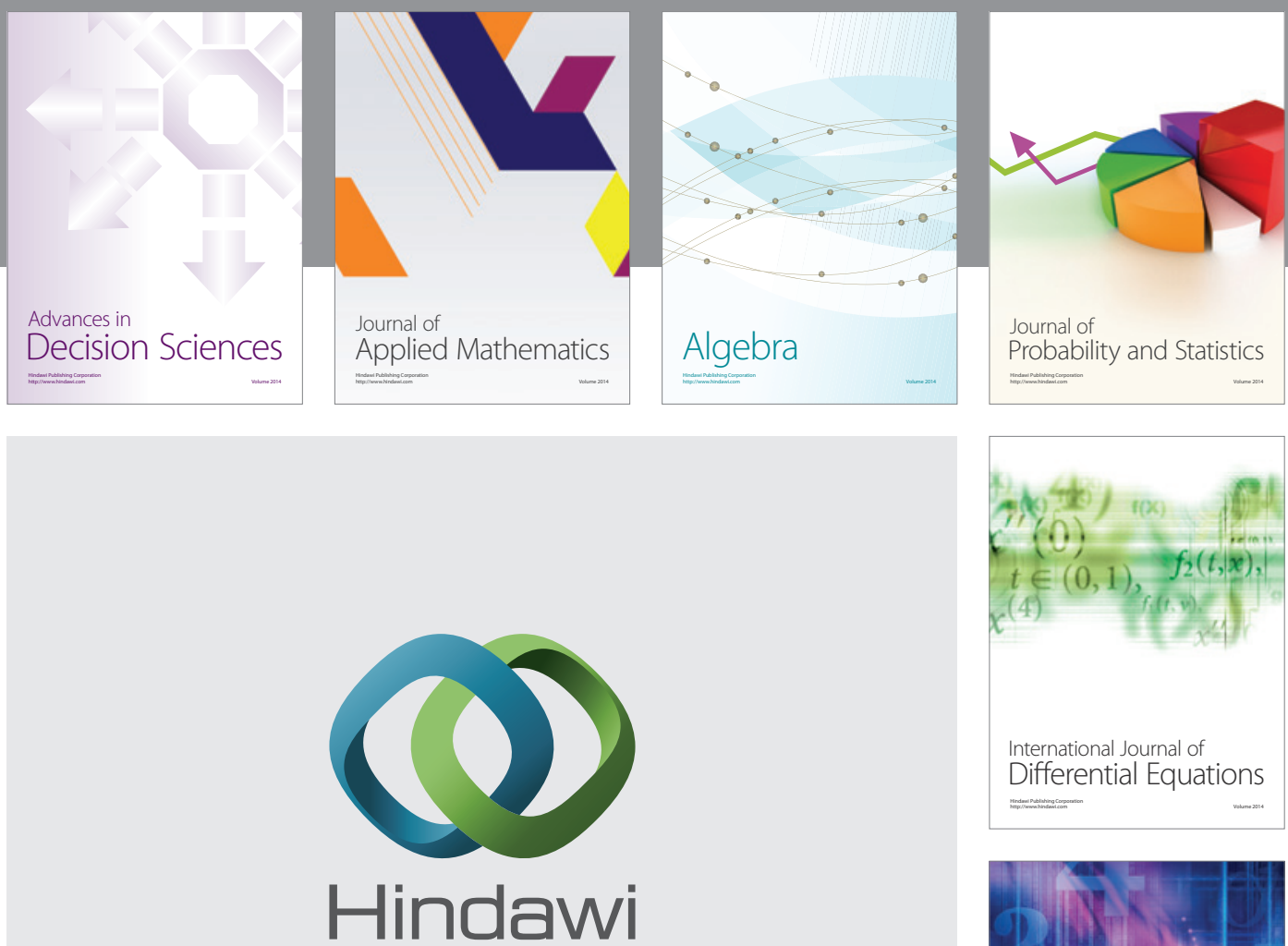

Submit your manuscripts at http://www.hindawi.com
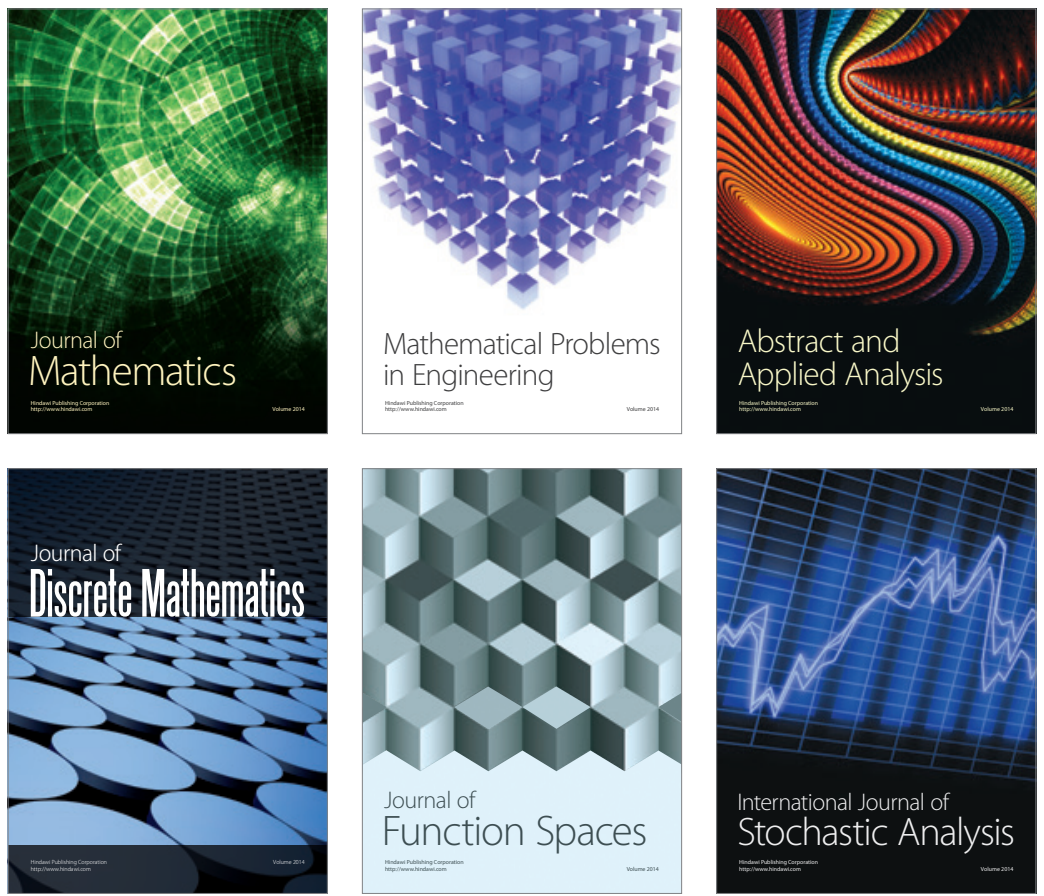

Journal of

Function Spaces

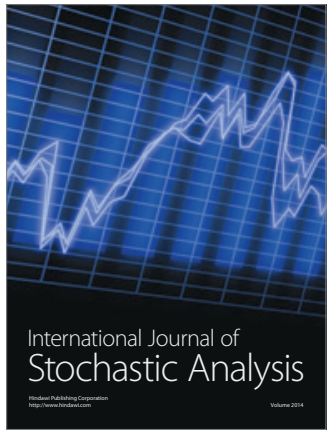

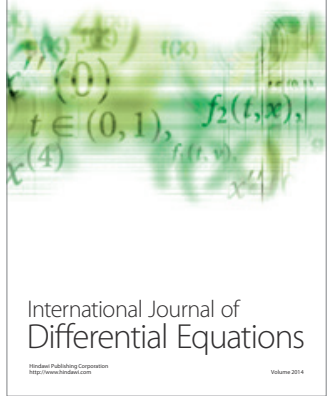
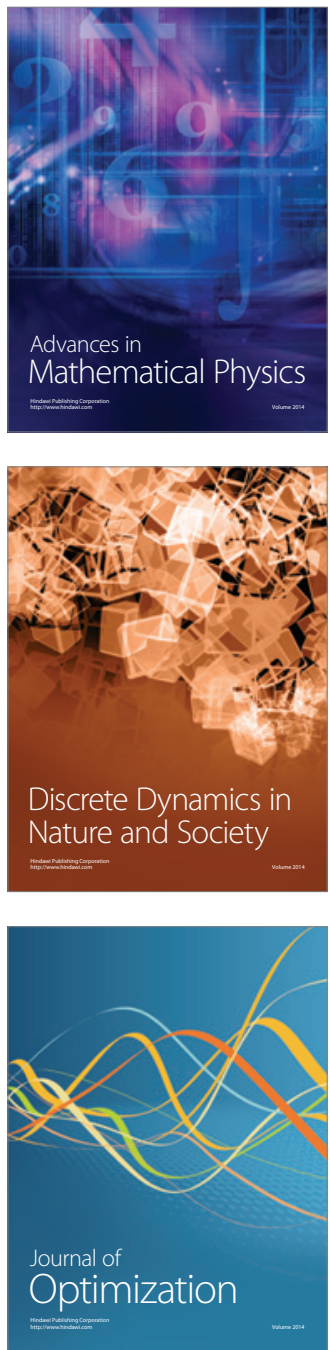